Homology, Homotopy and Applications, vol.5(1), 2003, pp.1-48

\title{
CATEGORY OF $A_{\infty}$-CATEGORIES
}

\section{VOLODYMYR LYUBASHENKO}

\author{
(communicated by James Stasheff)
}

\begin{abstract}
We define natural $A_{\infty}$-transformations and construct $A_{\infty}$-category of $A_{\infty}$-functors. The notion of non-strict units in an $A_{\infty}$-category is introduced. The 2-category of (unital) $A_{\infty}$-categories, (unital) functors and transformations is described.
\end{abstract}

The study of higher homotopy associativity conditions for topological spaces began with Stasheff's article [Sta63, I]. In a sequel to this paper [Sta63, II] Stasheff defines also $A_{\infty}$-algebras and their homotopy-bar constructions. These algebras and their applications to topology were actively studied, for instance, by Smirnov [Smi80] and Kadeishvili [Kad80, Kad82]. We adopt some notations of Getzler and Jones [GJ90], which reduce the number of signs in formulas. The notion of an $A_{\infty}$-category is a natural generalization of $A_{\infty}$-algebras. It arose in connection with Floer homology in Fukaya's work [Fuk93, Fuk] and was related by Kontsevich to mirror symmetry [Kon95]. See Keller [Kel01] for a survey on $A_{\infty}$-algebras and categories.

In the present article we show that given two $A_{\infty}$-categories $\mathcal{A}$ and $\mathcal{B}$, one can construct a third $A_{\infty}$-category $A_{\infty}(\mathcal{A}, \mathcal{B})$ whose objects are $A_{\infty}$-functors $f: \mathcal{A} \rightarrow \mathcal{B}$, and morphisms are natural $A_{\infty}$-transformations between such functors. This result was also obtained by Fukaya [Fuk] and by Kontsevich and Soibelman [KS], independently and, apparently, earlier. We describe compositions between such categories of $A_{\infty}$-functors, which would allow us to construct a 2-category of unital $A_{\infty}$-categories. The latter notion is our generalization of strictly unital $A_{\infty}$-categories (cf. Keller [Kel01]). We also discuss unit elements in unital $A_{\infty}$-categories, unital natural $A_{\infty}$-transformations, and unital $A_{\infty}$-functors.

Plan of the article with comments and explanations. The first section describes some notation, sign conventions, composition convention, etc. used in the article. The ground commutative ring $\mathbb{k}$ is not assumed to be a field. This is suggested by the development of homological algebra in [Dri02]. Working over a ring

The research was supported in part by grant 01.07/132 of State Fund for Fundamental Research of Ukraine

Received June 5, 2002, revised December 24, 2002; published on January 27, 2003.

2000 Mathematics Subject Classification: 18D05, 18D20, 18G55, 57T30

Key words and phrases: $A_{\infty}$-categories, $A_{\infty}$-functors, $A_{\infty}$-transformations, unit $A_{\infty}$-transformation, 2-category

(C) 2003, Volodymyr Lyubashenko. Permission to copy for private use granted. 
$\mathbb{k}$ instead of a field has strong consequences. For instance, one may not hope for Kadeishvili's theorem on minimal models [Kad82] to hold for all $A_{\infty}$-algebras over $\mathbb{k}$.

In the second section we recall or give definitions of the main objects. A $\mathbb{k}$-quiver is such a graph that the set of arrows (morphisms) between two vertices (objects) is a $\mathbb{k}$-module (Definition 2.1). We view quivers as categories without multiplication and units. Cocategories are $\mathbb{k}$-quivers and $\mathbb{k}$-coalgebras with a matrix type decomposition into $\mathbb{k}$-submodules, indexed by pairs of objects (Definition 2.2 ). $A_{\infty}$-categories are defined as a special kind of differential graded cocategories - the ones of the form of the tensor cocategory $T \mathcal{A}$ of a $\mathbb{k}$-quiver $\mathcal{A}$ (Definition 2.3). $A_{\infty}$-functors are homomorphisms of cocategories that commute with the differential (Definition 2.4). $A_{\infty}$-transformations between $A_{\infty}$-functors are defined as coderivations (Definition 2.6). They seem to make $A_{\infty}$-category theory closer to ordinary category theory. Notice, however, that $A_{\infty}$-transformations are analogs of transformations between ordinary functors, which do not satisfy the naturality condition. Natural $A_{\infty}$-transformations are introduced in Definition 6.4. $A_{\infty}$-functors and $A_{\infty}$-transformations are determined by their components.

In the third section we study tensor products of cocategories and homomorphisms between them (Section 3.2). We concentrate on homomorphisms from the tensor product of tensor cocategories to another tensor cocategory. Given $\mathbb{k}$-quivers $\mathcal{A}$ and $\mathcal{B}$, we consider another $\mathbb{k}$-quiver $\operatorname{Coder}(\mathcal{A}, \mathcal{B})$, whose objects are the cocategory homomorphisms $T \mathcal{A} \rightarrow T \mathcal{B}$ and morphisms are coderivations (Section 3.2). We construct a cocategory homomorphism $\alpha: T \mathcal{A} \otimes T \operatorname{Coder}(\mathcal{A}, \mathcal{B}) \rightarrow T \mathcal{B}$ (Corollary 3.3 to Proposition 3.1), based on a map $\theta: T \operatorname{Coder}(\mathcal{A}, \mathcal{B}) \rightarrow \operatorname{Hom}_{\mathbb{k}}(T \mathcal{A}, T \mathcal{B})(3.0 .1)$. The homomorphism $\alpha$ is universal (Proposition 3.4), in other words, $T \operatorname{Coder}(\mathcal{A}, \mathcal{B})$ is the inner hom-object $\operatorname{Hom}(T \mathcal{A}, T \mathcal{B})$ in the monoidal category generated by tensor cocategories.

This universality is exploited in the fourth section in order to show that the category of tensor cocategories is enriched in the monoidal category of graded cocategories. That is: there exists an associative unital multiplication $M: T \operatorname{Coder}(\mathcal{A}, \mathcal{B}) \otimes$ $T \operatorname{Coder}(\mathcal{B}, \mathcal{C}) \rightarrow T \operatorname{Coder}(\mathcal{A}, \mathcal{C})$, which is a cocategory homomorphism (Proposition 4.1). Its explicit description uses the map $\theta$.

The fifth section extends the results of the third section to differential graded tensor cocategories, that is, to $A_{\infty}$-categories. With two $A_{\infty}$-categories $\mathcal{A}, \mathcal{B}$ is associated a third $A_{\infty}$-category $A_{\infty}(\mathcal{A}, \mathcal{B})$ (Proposition 5.1). Its objects are $A_{\infty}$-functors $\mathcal{A} \rightarrow \mathcal{B}$, and its morphisms are coderivations. To reduce the number of signs in the theory we prefer to work with grading of a graded $\mathbb{k}$-quiver or $A_{\infty}$-category $\mathcal{A}$ shifted by $1: s \mathcal{A}=\mathcal{A}[1]$. In this notation the quiver $A_{\infty}(\mathcal{A}, \mathcal{B})$ is a full subquiver of the quiver $s^{-1} \operatorname{Coder}(s \mathcal{A}, s \mathcal{B})$. The proof of Proposition 5.1 consists of constructing a differential $B$ in the tensor cocategory of $A_{\infty}(\mathcal{A}, \mathcal{B})$. The explicit formula (5.1.3) for $B$ uses the map $\theta$. A cocategory homomorphism from a tensor product of differential tensor cocategories to a single such cocategory is called an $A_{\infty}$-functor in the generalized sense (Section 5.3). Restricting the cocategory homomorphism of Corollary 3.3 we get a homomorphism of differential graded cocategories $T s \mathcal{A} \otimes T s A_{\infty}(\mathcal{A}, \mathcal{B}) \rightarrow T s \mathcal{B}=T(s \mathcal{B})$ (Corollary 5.4). Its universality 
(Proposition 5.5) may be interpreted as $T s A_{\infty}(\mathcal{A}, \mathcal{B})$ being the inner hom-object $\operatorname{Hom}(T s \mathcal{A}, T s \mathcal{B})$ in the monoidal category generated by differential graded tensor cocategories.

This universality is used in the sixth section to show that the category of $A_{\infty}$-categories is enriched in the monoidal category of differential graded cocategories. Namely, the multiplication $M$ of Proposition 4.1 restricted to $M: T s A_{\infty}(\mathcal{A}, \mathcal{B}) \otimes$ $T s A_{\infty}(\mathcal{B}, \mathcal{C}) \rightarrow T s A_{\infty}(\mathcal{A}, \mathcal{C})$ is an $A_{\infty}$-functor, that is, it commutes with the differential (equation (6.1.1)). By universality (Proposition 5.5) $M$ corresponds to a unique $A_{\infty}$-functor

$$
A_{\infty}\left(\mathcal{A},{ }_{-}\right): A_{\infty}(\mathcal{B}, \mathcal{C}) \rightarrow A_{\infty}\left(A_{\infty}(\mathcal{A}, \mathcal{B}), A_{\infty}(\mathcal{A}, \mathcal{C})\right) .
$$

We prove that it is strict and describe it in Proposition 6.2. Natural $A_{\infty}$-transformations are defined as cycles in the differential graded quiver of all $A_{\infty}$-transformations (Definition 6.4).

Identifying cohomologous natural $A_{\infty}$-transformations (that is, considering cohomology of the quiver of $A_{\infty}$-transformations) in the seventh section, we get a non-2-unital 2-category $A_{\infty}$, whose objects are $A_{\infty}$-categories, 1-morphisms are $A_{\infty}$-functors, and 2-morphisms are equivalence classes of natural $A_{\infty}$-transformations. Here non-2-unital means that unit 2-morphisms are missing in the 2-category $A_{\infty}$. However, unit 1-morphisms are present - the identity $A_{\infty}$-functors. Before constructing $A_{\infty}$ we construct a non-2-unital 2-category $\mathcal{K} A_{\infty}$ enriched in $\mathcal{K}$ - the homotopy category of differential graded complexes of $\mathbb{k}$-modules (Proposition 7.1). Morphisms of $\mathcal{K}$ are chain maps modulo homotopy. The notion of 2-category enriched in a symmetric monoidal category is discussed in Appendix A. The idea of the construction is that the binary operation becomes strictly associative if homotopic chain maps are identified. Similarly with other identities in a 2-category. The non-2-unital 2-category $A_{\infty}$ is obtained from $\mathcal{K} A_{\infty}$ by taking the 0 -th cohomology.

$A_{\infty}$-categories are analogs of non-unital categories - categories without unit morphisms. We define a unital $A_{\infty}$-category $\mathcal{C}$ so that its cohomology $H \bullet(\mathcal{C})$ is a unital category, and for any representative $1_{X} \in \mathcal{C}^{0}(X, X)$ of the unit class $\left[1_{X}\right] \in H^{0}(\mathcal{C}(X, X))$ the binary compositions with $1_{X}$ are homotopic to identity as chain maps $\mathrm{C}(X, Y) \rightarrow \mathrm{C}(X, Y)$ or $\mathrm{e}(Y, X) \rightarrow \mathfrak{C}(Y, X)$ (Definition 7.3, Lemma 7.4). We prove that for a unital $A_{\infty}$-category there exists a natural $A_{\infty}$-transformation $\mathbf{i}^{\mathcal{C}}: \operatorname{id}_{\mathcal{C}} \rightarrow \operatorname{id}_{\mathcal{C}}: \mathcal{C} \rightarrow \mathcal{C}$ of the identity functor, whose square is equivalent to $\mathbf{i}^{\mathrm{e}}$ (Proposition 7.5). It is called a unit transformation of $\mathcal{C}$ (Definition 7.6) and, indeed, it is a unit 2-morphism in the 2-category $A_{\infty}$. Moreover, $f \mathbf{i}^{\mathrm{e}}: f \rightarrow f$ is a unit 2 -morphism of an $A_{\infty}$-functor $f: \mathcal{A} \rightarrow \mathcal{C}$ (Corollary 7.9). The unit transformation $\mathbf{i}^{\mathrm{e}}$ is determined uniquely up to an equivalence (Corollary 7.10). If $\mathcal{C}$ is unital, then $A_{\infty}(\mathcal{A}, \mathrm{C})$ is unital as well (Proposition 7.7).

The full 2-subcategory $\mathcal{K}^{u} A_{\infty}$ (resp. ${ }^{u} A_{\infty}$ ) of $\mathcal{K} A_{\infty}$ (resp. $A_{\infty}$ ), whose objects are unital $A_{\infty}$-categories, and 1-morphisms are all $A_{\infty}$-functors, is 2 -unital by Corollary 7.11 (resp. Corollary 7.12). Since ${ }^{u} A_{\infty}$ is an ordinary 2-category, the meaning of the statements 'a natural $A_{\infty}$-transformation is invertible' and 'an $A_{\infty}$-functor is an equivalence' is clear (Corollary 7.12). We show in Proposition 7.15 that a natural $A_{\infty}$-transformation is invertible if and only if its 0 -th component is invertible modulo boundary (in the sense of Section 7.13). The binary composition with a cycle 
invertible modulo boundary is homotopy invertible (Lemma 7.14).

In the eighth section we discuss unital $A_{\infty}$-functors (between unital $A_{\infty}$-categories). Their first components map unit elements into unit elements modulo boundary (Definition 8.1). For a unital functor $f: \mathcal{A} \rightarrow \mathcal{B}$ we have an equivalence of natural $A_{\infty}$-transformations $\mathbf{i}^{\mathcal{A}} f \equiv f \mathbf{i}^{\mathcal{B}}$ (Proposition 8.2). An $A_{\infty}$-functor isomorphic to a unital $A_{\infty}$-functor is unital as well (8.2.4). Unital $A_{\infty}$-categories, unital $A_{\infty}$-functors and equivalence classes of natural $A_{\infty}$-transformations form a 2-category $A_{\infty}^{u} \subset{ }^{u} A_{\infty}$ (Definition 8.3), which is a close analog of the 2-category of usual categories. The construction of $A_{\infty}^{u}$ is the main point of the article. It is needed for developing a theory of free $A_{\infty}$-categories, since it is expected that their universality properties are formulated in the language of 2-categories.

There is a forgetful 2-functor $\mathrm{k}: A_{\infty}^{u} \rightarrow \mathcal{K}$-Cat, which takes a unital $A_{\infty}$-category into the same differential quiver equipped with the binary composition, viewed as a $\mathcal{K}$-category (Proposition 8.6). The 2 -functor k reduces a unital $A_{\infty}$-functor to its first component and a natural $A_{\infty}$-transformation to its 0 -th component. It turns out that many properties of an $A_{\infty}$-functor are determined by its first component and many properties of a natural $A_{\infty}$-transformation are determined by its 0 -th component. For instance, if the first component of an $A_{\infty}$-functor $\phi$ is homotopy invertible, then any natural $A_{\infty}$-transformation $y: f \phi \rightarrow g \phi$ is equivalent to $t \phi$ for a unique (up to equivalence) natural $A_{\infty}$-transformation $t: f \rightarrow g$ (Cancellation Lemma 8.7). If an $A_{\infty}$-functor $\phi: \mathcal{C} \rightarrow \mathcal{B}$ to a unital $A_{\infty}$-category $\mathcal{B}$ has homotopy invertible first component, and each object of $\mathcal{B}$ is "isomorphic modulo boundary" to an object from $\phi(\mathrm{Ob} \mathcal{C})$, then $\phi$ is a unital equivalence, and $\mathcal{C}$ is unital (Theorem 8.8). An equivalence between unital $A_{\infty}$-categories is always unital (Corollary 8.9), which is not an immediate consequence of definitions.

As a first example of a unital $A_{\infty}$-category we list strictly unital $A_{\infty}$-categories (Section 8.11), which is a well-known notion. Other examples of unital $A_{\infty}$-categories are obtained via Theorem 8.8. For instance, if an $A_{\infty}$-functor $\phi: \mathcal{C} \rightarrow \mathcal{B}$ to a strictly unital $A_{\infty}$-category $\mathcal{B}$ is invertible, then $\mathcal{C}$ is unital (Section 8.12 ). We stress again that taking the 0 -th cohomology of a unital $A_{\infty}$-category $\mathcal{C}$ we get a $\mathbb{k}$-linear category $H^{0}(\mathcal{C})$. This $H^{0}$ can be viewed as a 2 -functor (Section 8.13).

In Appendix A we define 2-categories enriched in a symmetric monoidal category. Non-2-unital 2-categories are described in Definition Appendix A.2. 2-unital (usual) 2-categories admit a concise Definition Appendix A.1 and an expanded Definition Appendix A.3+Appendix A.2.

In Appendix B we prove that the cone of a homotopical isomorphism is contractible.

\section{Conventions}

We fix a universe $\mathscr{U}[$ GV73, Sections 0,1], [Bou73]. Many classes and sets in this paper will mean $\mathscr{U}$-small sets, even if not explicitly mentioned.

$\mathbb{k}$ denotes a ( $\mathscr{U}$-small) unital associative commutative ring. By abuse of notation it denotes also a chain complex, whose 0 -th component is $\mathbb{k}$, and the other components vanish. A $\mathbb{k}$-module means a $\mathscr{U}$-small $\mathbb{k}$-module. The tensor product $\otimes$ 
usually means $\otimes_{\mathbb{k}}$ - the tensor product of graded $\mathbb{k}$-modules. It turns the category of graded $\mathbb{k}$-modules into a closed monoidal category. We will use its standard symmetry $c: x \otimes y \mapsto(-)^{x y} y \otimes x=(-)^{\operatorname{deg} x \cdot \operatorname{deg} y} y \otimes x$. This paper contains many signs, and everywhere we abbreviate the usual $(-1)^{(\operatorname{deg} x)(\operatorname{deg} y)}$ to $(-)^{x y}$. Similarly, $(-)^{x}$ means $(-1)^{\operatorname{deg} x}$, or, simply, $(-1)^{x}$, if $x$ is an integer.

It is easy to understand the line

$$
\mathcal{A}\left(X_{0}, X_{1}\right) \otimes_{\mathbb{k}} \mathcal{A}\left(X_{1}, X_{2}\right) \otimes_{\mathbb{k}} \cdots \otimes_{\mathbb{k}} \mathcal{A}\left(X_{n-1}, X_{n}\right),
$$

and it is much harder to understand the order in

$$
\mathcal{A}\left(X_{n-1}, X_{n}\right) \otimes_{\mathbb{k}} \cdots \otimes_{\mathbb{k}} \mathcal{A}\left(X_{1}, X_{2}\right) \otimes_{\mathbb{k}} \mathcal{A}\left(X_{0}, X_{1}\right) .
$$

That is why we use the right operators: the composition of two maps (or morphisms) $f: X \rightarrow Y$ and $g: Y \rightarrow Z$ is denoted by $f g: X \rightarrow Z$. A map is written on elements as $f: x \mapsto x f=(x) f$. However, these conventions are not used systematically, and $f(x)$ might be used instead.

When $f, g: X \rightarrow Y$ are chain maps, $f \sim g$ means that they are homotopic. We denote by $\mathcal{K}$ the category of differential graded $\mathbb{k}$-modules, whose morphisms are chain maps modulo homotopy. A complex of $\mathbb{k}$-modules $X$ is called contractible if $\operatorname{id}_{X} \sim 0$, in other words, if $X$ is isomorphic to 0 in $\mathcal{K}$.

If $C$ is a $\mathbb{Z}$-graded $\mathbb{k}$-module, then its suspension $s C=C[1]$ is the same $\mathbb{k}$-module with the shifted grading $(s C)^{d}=C^{d+1}$. The "identity" map $C \rightarrow s C$ of degree -1 is also denoted by $s$. We follow the Getzler-Jones sign conventions [GJ90], which include the idea to apply operations to complexes with shifted grading, and Koszul's rule:

$$
(x \otimes y)(f \otimes g)=(-)^{y f} x f \otimes y g=(-1)^{\operatorname{deg} y \cdot \operatorname{deg} f} x f \otimes y g .
$$

It takes its origin in Koszul's note [Kos47]. The main notions of graded algebra were given their modern names in H. Cartan's note [Car48]. See Boardman [Boa66] for operad-like approach to signs as opposed to closed symmetric monoidal category picture of Mac Lane [Mac63] (standard sign commutation rule). Combined together, these sign conventions make the number of signs in this paper tolerable.

If $u: A \rightarrow C, a \mapsto a u$, is a chain map, its cone is the complex Cone $(u)=C \oplus A[1]$, $\operatorname{Cone}^{k}(u)=C^{k} \oplus A^{k+1}$, with the differential $(c, a) d=\left(c d^{C}+a u, a d^{A[1]}\right)=\left(c d^{C}+\right.$ $\left.a u,-a d^{A}\right)$.

\section{2. $A_{\infty}$-categories, $A_{\infty}$-functors and $A_{\infty}$-transformations}

2.1 Definition (Quiver). A graded $\mathbb{k}$-quiver $\mathcal{A}$ consists of the following data: a class of objects $\operatorname{Ob} \mathcal{A}$ (a $\mathscr{U}$-small set); a $\mathbb{Z}$-graded $\mathbb{k}$-module $\mathcal{A}(X, Y)=\operatorname{Hom}_{\mathcal{A}}(X, Y)$ for each pair of objects $X, Y$. A morphism of $\mathbb{k}$-quivers $f: \mathcal{A} \rightarrow \mathcal{B}$ is given by a map $f: \operatorname{Ob} \mathcal{A} \rightarrow \mathrm{Ob} \mathcal{B}, X \mapsto X f$ and by a $\mathbb{k}$-linear map $\mathcal{A}(X, Y) \rightarrow \mathcal{B}(X f, Y f)$ for each pair of objects $X, Y$ of $\mathcal{A}$.

To a given graded $\mathbb{k}$-quiver $\mathcal{A}$ we associate another graded $\mathbb{k}$-quiver - its tensor coalgebra $T \mathcal{A}$, which has the same class of objects as $\mathcal{A}$. For each sequence $\left(X_{0}, X_{1}, X_{2}, \ldots, X_{n}\right)$ of objects of $\mathcal{A}$ there is the $\mathbb{Z}$-graded $\mathbb{k}$-module $T^{n} \mathcal{A}=$ 
$\mathcal{A}\left(X_{0}, X_{1}\right) \otimes_{\mathbb{k}} \mathcal{A}\left(X_{1}, X_{2}\right) \otimes_{\mathbb{k}} \cdots \otimes_{\mathbb{k}} \mathcal{A}\left(X_{n-1}, X_{n}\right)$. For the sequence $\left(X_{0}\right)$ with $n=0$ it reduces to $T^{0} \mathcal{A}=\mathbb{k}$ in degree 0 . The graded $\mathbb{k}$-module $T \mathcal{A}(X, Y)=\oplus_{n \geqslant 0} T^{n} \mathcal{A}$ is the sum of the above modules over all sequences with $X_{0}=X, X_{n}=Y$. The quiver $T \mathcal{A}$ is equipped with the cut comultiplication $\Delta: T \mathcal{A}(X, Y) \rightarrow$ $\oplus_{Z \in \mathrm{Ob} \mathcal{A}} T \mathcal{A}(X, Z) \bigotimes_{\mathbb{k}} T \mathcal{A}(Z, Y), h_{1} \otimes h_{2} \otimes \cdots \otimes h_{n} \mapsto \sum_{k=0}^{n} h_{1} \otimes \cdots \otimes h_{k} \otimes h_{k+1} \otimes$ $\cdots \otimes h_{n}$, and the counit $\varepsilon=\left(T \mathcal{A}(X, Y) \stackrel{\mathrm{pr}_{0}}{\longrightarrow} T^{0} \mathcal{A}(X, Y) \rightarrow \mathbb{k}\right)$, where the last map is id $\operatorname{li}_{\mathbb{k}}$ if $X=Y$, or 0 if $X \neq Y$ (and $T^{0} \mathcal{A}(X, Y)=0$ ). For this article it is the main example of the following notion:

2.2 Definition (Cocategory). A graded cocategory $\mathcal{C}$ is a graded $\mathbb{k}$-quiver $\mathcal{C}$, equipped with a comultiplication - a $\mathbb{k}$-linear map $\Delta_{X, Y}^{Z}: \mathcal{C}(X, Y) \rightarrow \mathcal{C}(X, Z) \otimes_{\mathrm{k}}$ $\mathcal{C}(Z, Y)$ of degree 0 for all triples $X, Y, Z$ of objects of $\mathcal{C}$, and with a counit - a $\mathbb{k}$-linear map $\varepsilon_{X}: \mathcal{C}(X, X) \rightarrow \mathbb{k}$ of degree 0 for all objects $X$ of $\mathcal{C}$, such that the usual associativity equations and two counit equation hold.

Associated to each graded cocategory $\mathcal{C}$ is a graded $\mathbb{k}$-coalgebra $C=\oplus_{X, Y \in \mathrm{Ob}} \mathcal{e}(X, Y)$. Vice versa, to a graded $\mathbb{k}$-coalgebra, decomposed like that into $\mathbb{k}$-submodules $\mathrm{C}(X, Y), X, Y \in \mathrm{Ob} \mathcal{C}$, for some $\mathscr{U}$-small set $\mathrm{Ob} \mathcal{C}$, so that $\Delta(\mathcal{C}(X, Y)) \subset \oplus_{Z \in \mathrm{Ob}} \mathcal{e} \mathrm{e}(X, Z) \otimes_{\mathbb{k}} \mathcal{C}(Z, Y)$ for all pairs $X, Y$ of objects of $\mathcal{C}$, and $\varepsilon(\mathcal{C}(X, Y))=0$ for $X \neq Y$, we associate a graded cocategory.

This interpretation allows one to define a cocategory homomorphism $f: \mathcal{C} \rightarrow \mathcal{D}$ as a particular case of a coalgebra homomorphism: a map $f: \mathrm{Ob} \mathcal{C} \rightarrow \mathrm{Ob} \mathcal{D}$, and $\mathbb{k}$-linear maps $\mathcal{C}(X, Y) \rightarrow \mathcal{D}(X f, Y f)$ for all pairs of objects $X, Y$ of $\mathcal{A}$, compatible with comultiplication and counit. Given cocategory homomorphisms $f, g: \mathcal{C} \rightarrow \mathcal{D}$ we say that a system of $\mathbb{k}$-linear maps $r: \mathcal{C}(X, Y) \rightarrow \mathcal{D}(X f, Y g), X, Y \in \mathrm{Ob} \mathcal{C}$ is an $(f, g)$-coderivation, if the equation $r \Delta=\Delta(f \otimes r+r \otimes g)$ holds.

In particular, these definitions apply to the tensor coalgebras $T s \mathcal{A}=T(s \mathcal{A})$ of (suspended) $\mathbb{k}$-quivers $s \mathcal{A}$. In this case cocategory homomorphisms and coderivations have a special form as we shall see below.

2.3 Definition ( $A_{\infty}$-category, Kontsevich [Kon95]). An $A_{\infty}$-category $\mathcal{A}$ consists of the following data: a graded $\mathbb{k}$-quiver $\mathcal{A}$; a differential $b: T s \mathcal{A} \rightarrow T s \mathcal{A}$ of degree 1 , which is a $(1,1)$-coderivation, such that $\left(T^{0} s \mathcal{A}\right) b=0$.

The definition of a $(1,1)$-coderivation $b \Delta=\Delta(1 \otimes b+b \otimes 1)$ implies that a $\mathbb{k}$-quiver morphism $b$ is determined by a system of $\mathbb{k}$-linear maps $b \operatorname{pr}_{1}: T s \mathcal{A} \rightarrow s \mathcal{A}$ with components of degree 1

$$
b_{n}: s \mathcal{A}\left(X_{0}, X_{1}\right) \otimes s \mathcal{A}\left(X_{1}, X_{2}\right) \otimes \cdots \otimes s \mathcal{A}\left(X_{n-1}, X_{n}\right) \rightarrow s \mathcal{A}\left(X_{0}, X_{n}\right), \quad n \geqslant 1,
$$

via the formula

$$
b_{k l}=\left(\left.b\right|_{T^{k} \mathcal{A}_{\mathcal{A}}}\right) \operatorname{pr}_{l}: T^{k} s \mathcal{A} \rightarrow T^{l} s \mathcal{A}, \quad b_{k l}=\sum_{\substack{r+n+t=k \\ r+1+t=l}} 1^{\otimes r} \otimes b_{n} \otimes 1^{\otimes t} .
$$

Notice that the last condition of the definition implies $b_{0}=0$. In particular, $b_{k 0}=0$, and $k<l$ implies $b_{k l}=0$. Since $b^{2}$ is a $(1,1)$-coderivation of degree 2, the equation 
$b^{2}=0$ is equivalent to its particular case $b^{2} \mathrm{pr}_{1}=0$, that is, for all $k>0$

$$
\sum_{r+n+t=k}\left(1^{\otimes r} \otimes b_{n} \otimes 1^{\otimes t}\right) b_{r+1+t}=0: T^{k} s \mathcal{A} \rightarrow s \mathcal{A} .
$$

Using another, more traditional, form of components of $b$ :

$$
m_{n}=\left(\mathcal{A}^{\otimes n} \stackrel{s^{\otimes n}}{\longrightarrow}(s \mathcal{A})^{\otimes n} \stackrel{b_{n}}{\longrightarrow} s \mathcal{A} \stackrel{s^{-1}}{\longrightarrow} \mathcal{A}\right)
$$

we rewrite (2.3.1) as follows:

$$
\sum_{r+n+t=k}(-)^{t+r n}\left(1^{\otimes r} \otimes m_{n} \otimes 1^{\otimes t}\right) m_{r+1+t}=0: T^{k} \mathcal{A} \rightarrow \mathcal{A} .
$$

Notice that this equation differs in sign from [Kel01], because we are using right operators!

2.4 Definition ( $A_{\infty}$-functor, e.g. Keller [Kel01]). An $A_{\infty}$-functor $f: \mathcal{A} \rightarrow \mathcal{B}$ consists of the following data: $A_{\infty}$-categories $\mathcal{A}$ and $\mathcal{B}$, a cocategory homomorphism $f: T s \mathcal{A} \rightarrow T s \mathcal{B}$ of degree 0 , which commutes with the differential $b$.

The definition of a cocategory homomorphism $f \Delta=\Delta(f \otimes f), f \varepsilon=\varepsilon$ implies that $f$ is determined by a map $f: \mathrm{Ob} \mathcal{A} \rightarrow \mathrm{Ob} \mathcal{B}, X \mapsto X f$ and a system of $\mathbb{k}$-linear maps $f \operatorname{pr}_{1}: T s \mathcal{A} \rightarrow s \mathcal{B}$ with components of degree 0

$$
f_{n}: s \mathcal{A}\left(X_{0}, X_{1}\right) \otimes s \mathcal{A}\left(X_{1}, X_{2}\right) \otimes \cdots \otimes s \mathcal{A}\left(X_{n-1}, X_{n}\right) \rightarrow s \mathcal{B}\left(X_{0} f, X_{n} f\right),
$$

$n \geqslant 1$, (note that $f_{0}=0$ ) via the formula

$$
f_{k l}=\left(\left.f\right|_{T^{k}{ } \mathcal{A}}\right) \operatorname{pr}_{l}: T^{k} s \mathcal{A} \rightarrow T^{l} s \mathcal{B}, \quad f_{k l}=\sum_{i_{1}+\cdots+i_{l}=k} f_{i_{1}} \otimes f_{i_{2}} \otimes \cdots \otimes f_{i_{l}} .
$$

In particular, $f_{00}=\mathrm{id}: \mathbb{k} \rightarrow \mathbb{k}$, and $k<l$ implies $f_{k l}=0$. Since $f b$ and $b f$ are both $(f, f)$-coderivations of degree 1 , the equation $f b=b f$ is equivalent to its particular case $f b \mathrm{pr}_{1}=b f \mathrm{pr}_{1}$, that is, for all $k>0$

$\sum_{l>0 ; i_{1}+\cdots+i_{l}=k}\left(f_{i_{1}} \otimes f_{i_{2}} \otimes \cdots \otimes f_{i_{l}}\right) b_{l}=\sum_{r+n+t=k}\left(1^{\otimes r} \otimes b_{n} \otimes 1^{\otimes t}\right) f_{r+1+t}: T^{k} s \mathcal{A} \rightarrow s \mathcal{B}$.

Using $m_{n}$ we rewrite (2.4.2) as follows:

$$
\begin{gathered}
\sum_{i_{1}+\cdots+i_{l}=k}^{l>0}(-)^{\sigma}\left(f_{i_{1}} \otimes f_{i_{2}} \otimes \cdots \otimes f_{i_{l}}\right) m_{l}= \\
\sum_{\substack{r+n+t=k \\
\sigma=\left(i_{2}-1\right)+2\left(i_{3}-1\right)+\cdots+(l-2)\left(i_{l-1}-1\right)+(l-1)\left(i_{l}-1\right) .}}\left(1^{\otimes r} \otimes m_{n} \otimes 1^{\otimes t}\right) f_{r+1+t}: T^{k} \mathcal{A} \rightarrow \mathcal{B},
\end{gathered}
$$

Notice that this equation differs in sign from [Kel01], because we are using right operators.

2.5 Example. An $A_{\infty}$-category with one object is called an $A_{\infty}$-algebra (Stasheff [Sta63]). An $A_{\infty}$-functor between $A_{\infty}$-algebras is called an $A_{\infty}$-homomorphism 
(Kadeishvili [Kad82]). These notions are psychologically easier to deal with, than the general case. The following notion of $A_{\infty}$-transformations also makes sense for $A_{\infty}$-algebras, however, such a context seems too narrow for it, because an $A_{\infty}$-transformation is an analog of a transformation between ordinary functors without the naturality condition. Needless to say, in ordinary category theory there is no reason to consider unnatural transformations. The reasons to do it for $A_{\infty}$-version are given in Section 5 .

2.6 Definition ( $A_{\infty}$-transformation). An $A_{\infty}$-transformation $r: f \rightarrow g: \mathcal{A} \rightarrow \mathcal{B}$ of degree $d$ (pre natural transformation in terms of [Fuk]) consists of the following data: $A_{\infty}$-categories $\mathcal{A}$ and $\mathcal{B} ; A_{\infty}$-functors $f, g: \mathcal{A} \rightarrow \mathcal{B}$; an $(f, g)$-coderivation $r: T s \mathcal{A} \rightarrow T s \mathcal{B}$ of degree $d$.

The definition of an $(f, g)$-coderivation $r \Delta=\Delta(f \otimes r+r \otimes g)$ implies that $r$ is determined by a system of $\mathbb{k}$-linear maps $r \operatorname{pr}_{1}: T s \mathcal{A} \rightarrow s \mathcal{B}$ with components of degree $d$

$$
r_{n}: s \mathcal{A}\left(X_{0}, X_{1}\right) \otimes s \mathcal{A}\left(X_{1}, X_{2}\right) \otimes \cdots \otimes s \mathcal{A}\left(X_{n-1}, X_{n}\right) \rightarrow s \mathcal{B}\left(X_{0} f, X_{n} g\right),
$$

$n \geqslant 0$, via the formula

$$
\begin{aligned}
& r_{k l}=\left(\left.r\right|_{T^{k} k_{\mathcal{A}}}\right) \operatorname{pr}_{l}: T^{k} s \mathcal{A} \rightarrow T^{l} s \mathcal{B}, \\
& r_{k l}=\sum_{\substack{q+1+t=l \\
i_{1}+\cdots+i_{q}+n+j_{1}+\cdots+j_{t}=k}} f_{i_{1}} \otimes \cdots \otimes f_{i_{q}} \otimes r_{n} \otimes g_{j_{1}} \otimes \cdots \otimes g_{j_{t}} .
\end{aligned}
$$

Note that $r_{0}$ is a system of $\mathbb{k}$-linear maps ${ }_{X} r_{0}: \mathbb{k} \rightarrow s \mathcal{B}(X f, X g), X \in \mathrm{Ob} \mathcal{A}$. In fact, the terms ' $A_{\infty}$-transformation' and 'coderivation' are synonyms.

In particular, $r_{0 l}$ vanishes unless $l=1$, and $r_{01}=r_{0}$. The component $r_{k l}$ vanishes unless $1 \leqslant l \leqslant k+1$.

2.7 Examples. 1) The restriction of an $A_{\infty}$-transformation $r$ to $T^{1}$ is

$$
\left.r\right|_{T^{1} s \mathcal{A}}=r_{1} \oplus\left[\left(f_{1} \otimes r_{0}\right)+\left(r_{0} \otimes g_{1}\right)\right],
$$

where $r_{1}: s \mathcal{A}(X, Y) \rightarrow s \mathcal{B}(X f, Y g)$,

$$
\begin{aligned}
& f_{1} \otimes r_{0}: s \mathcal{A}(X, Y)=s \mathcal{A}(X, Y) \otimes \mathbb{k} \stackrel{f_{1} \otimes r_{0}}{\longrightarrow} s \mathcal{B}(X f, Y f) \otimes s \mathcal{B}(Y f, Y g), \\
& r_{0} \otimes g_{1}: s \mathcal{A}(X, Y)=\mathbb{k} \otimes s \mathcal{A}(X, Y) \stackrel{r_{0} \otimes g_{1}}{\longrightarrow} s \mathcal{B}(X f, X g) \otimes s \mathcal{B}(X g, Y g) .
\end{aligned}
$$

2) The restriction of an $A_{\infty}$-transformation $r$ to $T^{2}$ is

$$
\begin{aligned}
&\left.r\right|_{T^{2} s \mathcal{A}}=r_{2} \oplus\left[\left(f_{2} \otimes r_{0}\right)+\right.\left.\left(f_{1} \otimes r_{1}\right)+\left(r_{1} \otimes g_{1}\right)+\left(r_{0} \otimes g_{2}\right)\right] \oplus \\
& \oplus\left[\left(f_{1} \otimes f_{1} \otimes r_{0}\right)+\left(f_{1} \otimes r_{0} \otimes g_{1}\right)+\left(r_{0} \otimes g_{1} \otimes g_{1}\right)\right],
\end{aligned}
$$


where $r_{2}: s \mathcal{A}(X, Y) \otimes s \mathcal{A}(Y, Z) \rightarrow s \mathcal{B}(X f, Z g)$,

$f_{2} \otimes r_{0}: s \mathcal{A}(X, Y) \otimes s \mathcal{A}(Y, Z) \otimes \mathbb{k} \rightarrow s \mathcal{B}(X f, Z f) \otimes s \mathcal{B}(Z f, Z g)$,

$f_{1} \otimes r_{1}: s \mathcal{A}(X, Y) \otimes s \mathcal{A}(Y, Z) \rightarrow s \mathcal{B}(X f, Y f) \otimes s \mathcal{B}(Y f, Z g)$,

$r_{1} \otimes g_{1}: s \mathcal{A}(X, Y) \otimes s \mathcal{A}(Y, Z) \rightarrow s \mathcal{B}(X f, Y g) \otimes s \mathcal{B}(Y g, Z g)$,

$r_{0} \otimes g_{2}: \mathbb{k} \otimes s \mathcal{A}(X, Y) \otimes s \mathcal{A}(Y, Z) \rightarrow s \mathcal{B}(X f, X g) \otimes s \mathcal{B}(X g, Z g)$,

$f_{1} \otimes f_{1} \otimes r_{0}: s \mathcal{A}(X, Y) \otimes s \mathcal{A}(Y, Z) \otimes \mathbb{k} \rightarrow s \mathcal{B}(X f, Y f) \otimes s \mathcal{B}(Y f, Z f) \otimes s \mathcal{B}(Z f, Z g)$,

$f_{1} \otimes r_{0} \otimes g_{1}: s \mathcal{A}(X, Y) \otimes \mathbb{k} \otimes s \mathcal{A}(Y, Z) \rightarrow s \mathcal{B}(X f, Y f) \otimes s \mathcal{B}(Y f, Y g) \otimes s \mathcal{B}(Y g, Z g)$,

$r_{0} \otimes g_{1} \otimes g_{1}: \mathbb{k} \otimes s \mathcal{A}(X, Y) \otimes s \mathcal{A}(Y, Z) \rightarrow s \mathcal{B}(X f, X g) \otimes s \mathcal{B}(X g, Y g) \otimes s \mathcal{B}(Y g, Z g)$.

The $\mathbb{k}$-module of $(f, g)$-coderivations $r$ is $\prod_{n=0}^{\infty} V_{n}$, where

$$
V_{n}=\prod_{X_{0}, \ldots, X_{n} \in \mathrm{Ob} \mathcal{A}} \operatorname{Hom}_{\mathbb{k}}\left(s \mathcal{A}\left(X_{0}, X_{1}\right) \otimes \cdots \otimes s \mathcal{A}\left(X_{n-1}, X_{n}\right), s \mathcal{B}\left(X_{0} f, X_{n} g\right)\right)
$$

is the graded $\mathbb{k}$-module of $n$-th components $r_{n}$. It is equipped with the differential $d: V_{n} \rightarrow V_{n}$, given by the following formula

$$
r_{n} d=r_{n} b_{1}-(-)^{r_{n}} \sum_{\alpha+1+\beta=n}\left(1^{\otimes \alpha} \otimes b_{1} \otimes 1^{\otimes \beta}\right) r_{n} .
$$

\section{Coderivations and cocategory homomorphisms}

Let $\mathcal{A}, \mathcal{B}$ be graded $\mathbb{k}$-quivers, and let $f^{0}, f^{1}, \ldots, f^{n}: T \mathcal{A} \rightarrow T \mathcal{B}$ be cocategory homomorphisms. Consider $n$ coderivations $r_{1}, \ldots, r_{n}$ as in

$$
f^{0} \stackrel{r^{1}}{\longrightarrow} f^{1} \stackrel{r^{2}}{\longrightarrow} \ldots f^{n-1} \stackrel{r^{n}}{\longrightarrow} f^{n}: T \mathcal{A} \rightarrow T \mathcal{B} .
$$

We construct the following system of $\mathbb{k}$-linear maps from these data: $\theta=\left(r^{1} \otimes \cdots \otimes\right.$ $\left.r^{n}\right) \theta: T \mathcal{A}(X, Y) \rightarrow T \mathcal{B}\left(X f^{0}, Y f^{n}\right)$ of degree $\operatorname{deg} r^{1}+\cdots+\operatorname{deg} r^{n}$. Its components $\theta_{k l}=\left.\theta\right|_{T^{k} \mathcal{A}} \operatorname{pr}_{l}: T^{k} \mathcal{A} \rightarrow T^{l} \mathcal{B}$ are given by the following formula

$$
\theta_{k l}=\sum f_{i_{1}^{0}}^{0} \otimes \cdots \otimes f_{i_{m_{0}}^{0}}^{0} \otimes r_{j_{1}}^{1} \otimes f_{i_{1}^{1}}^{1} \otimes \cdots \otimes f_{i_{m_{1}}^{1}}^{1} \otimes \cdots \otimes r_{j_{n}}^{n} \otimes f_{i_{1}^{n}}^{n} \otimes \cdots \otimes f_{i_{m_{n}}^{n}}^{n},
$$

where summation is taken over all terms with

$m_{0}+m_{1}+\cdots+m_{n}+n=l, \quad i_{1}^{0}+\cdots+i_{m_{0}}^{0}+j_{1}+i_{1}^{1}+\cdots+i_{m_{1}}^{1}+\cdots+j_{n}+i_{1}^{n}+\cdots+i_{m_{n}}^{n}=k$.

The component $\theta_{k l}$ vanishes unless $n \leqslant l \leqslant k+n$. If $n=0$, we set ()$\theta=f^{0}$. If $n=1$, the formula gives $\left(r^{1}\right) \theta=r^{1}$.

3.1 Proposition. For each $n \geqslant 0$ the map $\theta$ satisfies the equation

$$
\left(r^{1} \otimes r^{2} \otimes \cdots \otimes r^{n}\right) \theta \Delta=\Delta \sum_{k=0}^{n}\left(r^{1} \otimes \cdots \otimes r^{k}\right) \theta \otimes\left(r^{k+1} \otimes \cdots \otimes r^{n}\right) \theta .
$$

Proof. Let us write down the required equation using $\otimes$ to separate the two copies of $T \mathcal{B}$ in $T \mathcal{B} \otimes T \mathcal{B}$, and $\otimes$ to denote the multiplication in $T \mathcal{B}$. We have to prove that for each $n, x, y, z \geqslant 0$ 


$$
\begin{aligned}
& \left(T^{x} \mathcal{A} \stackrel{\left(r^{1} \otimes \cdots \otimes r^{n}\right) \theta_{x, y+z}}{\longrightarrow} T^{y+z} \mathcal{B} \stackrel{\Delta_{y z}}{\longrightarrow} T^{y} \mathcal{B} \bigotimes T^{z} \mathcal{B}\right) \\
= & \sum_{k=0}^{n} \sum_{u+v=x}\left(T^{x} \mathcal{A} \stackrel{\Delta_{u v}}{\longrightarrow} T^{u} \mathcal{A} \bigotimes T^{v} \mathcal{A} \stackrel{\left(r^{1} \otimes \cdots \otimes r^{k}\right) \theta_{u y} \otimes\left(r^{k+1} \otimes \cdots \otimes r^{n}\right) \theta_{v z}}{\longrightarrow} T^{y} \mathcal{B} \bigotimes T^{z} \mathcal{B}\right) .
\end{aligned}
$$

Substituting (3.0.1) for $\theta$ in the above equation we come to an identity, which is proved by inspection. Indeed, skipping all the intermediate steps, we get the following equation:

$$
\begin{aligned}
& \sum_{k=0}^{n} \sum_{\substack{m_{0}+m_{1}+\cdots+m_{k-1}+k-1<y \leqslant m_{0}+m_{1}+\cdots+m_{k}+k \\
m_{0}+\cdots+m^{0}+\cdots+i^{0} \\
i_{1}+j_{1}+i_{1}^{1}+\cdots+i^{1}}} \\
& f_{i_{1}^{0}}^{0} \otimes \cdots \otimes f_{i_{m_{0}}^{0}}^{0} \otimes \cdots \otimes r_{j_{k}}^{k} \otimes f_{i_{1}^{k}}^{k} \otimes \cdots \otimes f_{i_{w}^{k}}^{k} \bigotimes f_{i_{w+1}^{k}}^{k} \otimes \cdots \otimes f_{i_{m_{k}}^{k}}^{k} \otimes r_{j_{k+1}}^{k+1} \otimes \cdots \otimes f_{i_{1}^{n}}^{n} \otimes \cdots \otimes f_{i_{m_{n}}^{n}}^{n} \\
& =\sum_{k=0}^{n} \sum_{u+v=x} \sum_{\substack{m_{0}+m_{1}+\cdots+m_{k-1}+k+w=y \\
i_{1}^{0}+\cdots+i_{m_{0}}^{0}+\cdots+j_{k}+i_{1}^{k}+\cdots+i_{w}^{k}=u}} \sum_{\substack{t+m_{k+1}+\cdots+m_{n}+n-k=z \\
l_{1}+\cdots+l_{t}+j_{k+1}+\cdots+i_{1}^{n}+\cdots+i_{m_{n}}^{n}}} \\
& f_{i_{1}^{0}}^{0} \otimes \cdots \otimes f_{i_{m_{0}}^{0}}^{0} \otimes \cdots \otimes r_{j_{k}}^{k} \otimes f_{i_{1}^{k}}^{k} \otimes \cdots \otimes f_{i_{w}^{k}}^{k} \otimes f_{l_{1}}^{k} \otimes \cdots \otimes f_{l_{t}}^{k} \otimes r_{j_{k+1}}^{k+1} \otimes \cdots \otimes f_{i_{1}^{n}}^{n} \otimes \cdots \otimes f_{i_{m_{n}}^{n}}^{n} .
\end{aligned}
$$

In the left hand side $w$ denotes the expression $y-\left(m_{0}+m_{1}+\cdots+m_{k-1}+k\right)$ and lies in the interval $0 \leqslant w \leqslant m_{k}$. Identifying $m_{k}$ in the left hand side with $w+t$ in the right hand side, we deduce that the both sides are equal.

3.2. Cocategory homomorphisms. Graded $\mathbb{k}$-coalgebras form a symmetric monoidal category. The tensor product $C \otimes_{\mathbb{k}} D$ of $\mathbb{k}$-coalgebras $C, D$ is equipped with the comultiplication $C \otimes D \stackrel{\Delta \otimes \Delta}{\longrightarrow} C \otimes C \otimes D \otimes D \stackrel{1 \otimes c \otimes 1}{\longrightarrow} C \otimes D \otimes C \otimes D$, using the standard symmetry $c$ of graded $\mathbb{k}$-modules. Since graded cocategories are, in fact, graded coalgebras with a special decomposition, they also form a symmetric monoidal category gCoCat. If $\mathcal{C}$ and $\mathcal{D}$ are cocategories, then the class of objects of their tensor product $\mathcal{C} \otimes \mathcal{D}$ is $\mathrm{Ob} \mathcal{C} \times \mathrm{Ob} \mathcal{D}$, and $\mathcal{C} \otimes \mathcal{D}(X \times U, Y \times W)=\mathcal{C}(X, Y) \otimes_{\mathbb{k}} \mathcal{D}(U, W)$.

Let $\phi: T \mathcal{A} \otimes T \mathcal{C} \rightarrow T \mathcal{B}$ be a cocategory homomorphism of degree 0 . It is determined uniquely by its composition with $\mathrm{pr}_{1}$, that is, by a family $\phi \mathrm{pr}_{1}=$ $\left(\phi_{n m}\right)_{n, m \geqslant 0}, \phi_{n m}: T^{n} \mathcal{A} \otimes T^{m} \mathcal{C} \rightarrow \mathcal{B}, \phi_{00}=0$, with the same underlying map of objects $\mathrm{Ob} \mathcal{A} \times \mathrm{ObC} \rightarrow \mathrm{Ob} \mathcal{B}$. Indeed, for given families of composable arrows $f^{0} \stackrel{p^{1}}{\longrightarrow} f^{1} \stackrel{p^{2}}{\longrightarrow} \ldots f^{n-1} \stackrel{p^{n}}{\longrightarrow} f^{n}$ of $\mathcal{A}$ and $g^{0} \stackrel{t^{1}}{\longrightarrow} g^{1} \stackrel{t^{2}}{\longrightarrow} \ldots g^{m-1} \stackrel{t^{m}}{\longrightarrow} g^{m}$ of e we have 


$$
\begin{aligned}
& \left(p^{1} \otimes \cdots \otimes p^{n} \otimes t^{1} \otimes \cdots \otimes t^{m}\right) \phi \\
& =\sum_{\substack{i_{1}+\cdots+i_{k}=n \\
j_{1}+\cdots+j_{k}=m}}(-)^{\sigma}\left(p^{1} \otimes \cdots \otimes p^{i_{1}} \otimes t^{1} \otimes \cdots \otimes t^{j_{1}}\right) \phi_{i_{1} j_{1}} \\
& \quad \otimes\left(p^{i_{1}+1} \otimes \cdots \otimes p^{i_{1}+i_{2}} \otimes t^{j_{1}+1} \otimes \cdots \otimes t^{j_{1}+j_{2}}\right) \phi_{i_{2} j_{2}} \\
& \otimes \cdots \otimes\left(p^{i_{1}+\cdots+i_{k-1}+1} \otimes \cdots \otimes p^{i_{1}+\cdots+i_{k}} \otimes t^{j_{1}+\cdots+j_{k-1}+1} \otimes \cdots \otimes t^{j_{1}+\cdots+j_{k}}\right) \phi_{i_{k} j_{k}} .
\end{aligned}
$$

The sign depends on the parity of an integer

$$
\begin{aligned}
\sigma= & \left(t^{1}+\cdots+t^{j_{1}}\right)\left(p^{i_{1}+1}+\cdots+p^{i_{1}+\cdots+i_{k}}\right)+ \\
& \quad\left(t^{j_{1}+1}+\cdots+t^{j_{1}+j_{2}}\right)\left(p^{i_{1}+i_{2}+1}+\cdots+p^{i_{1}+\cdots+i_{k}}\right) \\
& +\cdots+\left(t^{j_{1}+\cdots+j_{k-2}+1}+\cdots+t^{j_{1}+\cdots+j_{k-1}}\right)\left(p^{i_{1}+\cdots+i_{k-1}+1}+\cdots+p^{i_{1}+\cdots+i_{k}}\right),
\end{aligned}
$$

where each coderivation has to be replaced with its degree. Recall that in our notation (Section 1) we abbreviate $(-1)^{(\operatorname{deg} t)(\operatorname{deg} p)}$ to $(-)^{t p}$. By definition the homomorphism $\phi$ satisfies the equation

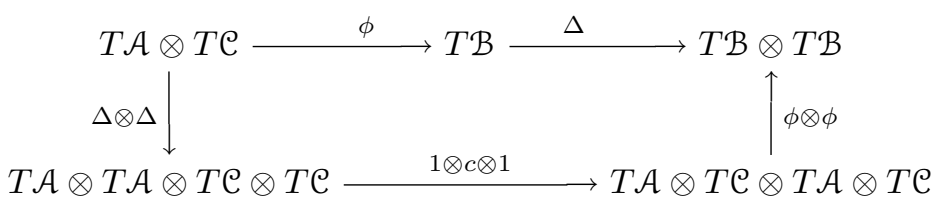

$T \mathcal{A} \otimes T \mathcal{A} \otimes T \mathcal{C} \otimes T \mathcal{C} \longrightarrow \frac{1 \otimes c \otimes 1}{\longrightarrow} T \mathcal{A} \otimes T \mathcal{C} \otimes T \mathcal{A} \otimes T \mathcal{C}$

Introduce $\mathbb{k}$-linear maps $\left(t^{1} \otimes \cdots \otimes t^{m}\right) \chi: T \mathcal{A} \rightarrow T \mathcal{B}$ by the formula $a\left[\left(t^{1} \otimes \cdots \otimes\right.\right.$ $\left.\left.t^{m}\right) \chi\right]=\left(a \otimes t^{1} \otimes \cdots \otimes t^{m}\right) \phi, a \in T \mathcal{A}$. Then the above equation is equivalent to

$$
\left(t^{1} \otimes t^{2} \otimes \cdots \otimes t^{m}\right) \chi \Delta=\Delta \sum_{k=0}^{m}\left(t^{1} \otimes \cdots \otimes t^{k}\right) \chi \otimes\left(t^{k+1} \otimes \cdots \otimes t^{m}\right) \chi .
$$

for all $m \geqslant 0$.

When $\mathcal{A}, \mathcal{B}$ are graded $\mathbb{k}$-quivers, we define a new $\mathbb{k}$-quiver $\operatorname{Coder}(\mathcal{A}, \mathcal{B})$, whose objects are cocategory homomorphisms $f: T \mathcal{A} \rightarrow T \mathcal{B}$. These homomorphisms are determined by a system $f \mathrm{pr}_{1}=\left(f_{n}\right)_{n \geqslant 1}$ of morphisms of $\mathbb{k}$-quivers $f_{n}: T^{n} \mathcal{A} \rightarrow \mathcal{B}$ of degree 0 with the same underlying map $\mathrm{Ob} \mathcal{A} \rightarrow \mathrm{Ob} \mathcal{B}$, see (2.4.1). The $\mathbb{k}$-module of morphisms between $f, g: T \mathcal{A} \rightarrow T \mathcal{B}$ consists of $(f, g)$-coderivations:

$[\operatorname{Coder}(\mathcal{A}, \mathcal{B})(f, g)]^{d}=\{r: T \mathcal{A} \rightarrow T \mathcal{B} \mid r \Delta=\Delta(f \otimes r+r \otimes g), \quad \operatorname{deg} r=d\}, \quad d \in \mathbb{Z}$. Such a coderivation $r$ is determined by a system of $\mathbb{k}$-linear maps $r \mathrm{pr}_{1}=\left(r_{n}\right)_{n \geqslant 0}$, $r_{n}: T^{n} \mathcal{A}(X, Y) \rightarrow \mathcal{B}(X f, Y g)$ of degree $d$ as in (2.6.1).

3.3 Corollary (to Proposition 3.1). A map $\alpha: T \mathcal{A} \otimes T \operatorname{Coder}(\mathcal{A}, \mathcal{B}) \rightarrow T \mathcal{B}$, $a \otimes r^{1} \otimes \cdots \otimes r^{n} \mapsto a\left[\left(r^{1} \otimes \cdots \otimes r^{n}\right) \theta\right]$, is a cocategory homomorphism of degree 0 .

Proof. Equation (3.1.1) means that equation (3.2.4) holds for $\chi=\theta$, which is equivalent to $(3.2 .3)$ for $\phi=\alpha, \mathcal{C}=\operatorname{Coder}(\mathcal{A}, \mathcal{B})$. 
3.4 Proposition. For any cocategory homomorphism $\phi: T \mathcal{A} \otimes T \mathrm{C}^{1} \otimes T \mathrm{C}^{2} \otimes$ $\cdots \otimes T \mathrm{C}^{q} \rightarrow T \mathcal{B}$ of degree 0 there is a unique cocategory homomorphism $\psi$ : $T \mathrm{C}^{1} \otimes T \mathrm{C}^{2} \otimes \cdots \otimes T \mathrm{C}^{q} \rightarrow T \operatorname{Coder}(\mathcal{A}, \mathcal{B})$ of degree 0 , such that

$$
\phi=\left(T \mathcal{A} \otimes T \mathcal{C}^{1} \otimes T \mathcal{C}^{2} \otimes \cdots \otimes T \mathcal{C}^{q} \stackrel{1 \otimes \psi}{\longrightarrow} T \mathcal{A} \otimes T \operatorname{Coder}(\mathcal{A}, \mathcal{B}) \stackrel{\alpha}{\longrightarrow} T \mathcal{B}\right) .
$$

Proof. Let us start with a simple case $q=1, \mathcal{C}=\mathcal{C}^{1}$. Each object $g$ of $\mathcal{C}$ induces a cocategory morphism $g \psi: a \mapsto(a \otimes g) \phi$. We set $\psi_{0}=0$. Each element $p \in \mathcal{C}(g, h)$ induces a coderivation $(p) \psi_{1}=p \psi: a \mapsto(a \otimes p) \phi$. Suppose that $\psi_{i}$ are already found for $0 \leqslant i<n$. Then we find $\psi_{n}$ from the sought identity $\chi=\psi \theta$. Namely, for $g^{0} \stackrel{p^{1}}{\longrightarrow} g^{1} \stackrel{p^{2}}{\longrightarrow} \ldots g^{n-1} \stackrel{p^{n}}{\longrightarrow} g^{n}$ we have to satisfy the identity

$\left(p^{1} \otimes \cdots \otimes p^{n}\right) \chi=\left(p^{1} \otimes \cdots \otimes p^{n}\right) \psi_{n}+\sum_{l=2}^{n} \sum_{i_{1}+\cdots+i_{l}=n}\left[\left(p^{1} \otimes \cdots \otimes p^{n}\right) \cdot\left(\psi_{i_{1}} \otimes \psi_{i_{2}} \otimes \cdots \otimes \psi_{i_{l}}\right)\right] \theta$,

which expresses $\psi$ via its components $\psi_{k}$. Notice that the unknown $\psi_{n}$ occurs only in the singled out summand, corresponding to $l=1$. The factors $\psi_{i}$ in the sum are already known, since $i<n$. So we define $\left(p^{1} \otimes \cdots \otimes p^{n}\right) \psi_{n}: T \mathcal{A} \rightarrow T \mathcal{B}$ as the difference of $\left(p^{1} \otimes \cdots \otimes p^{n}\right) \chi$ and the sum in the right hand side. Assume that $\psi$ is a cocategory homomorphism up to the level $n$, that is,

$$
\left(p^{1} \otimes \cdots \otimes p^{m}\right) \psi=\sum_{l=1}^{m} \sum_{i_{1}+\cdots+i_{l}=m}\left(p^{1} \otimes \cdots \otimes p^{m}\right) \cdot\left(\psi_{i_{1}} \otimes \psi_{i_{2}} \otimes \cdots \otimes \psi_{i_{l}}\right)
$$

for all $0 \leqslant m \leqslant n$. Taking into account equations (3.1.1), we see that (3.2.4) is equivalent to an equation of the form

$$
\left(p^{1} \otimes \cdots \otimes p^{n}\right) \psi_{n} \Delta=\Delta\left[g^{0} \psi \otimes\left(p^{1} \otimes \cdots \otimes p^{n}\right) \psi_{n}+\left(p^{1} \otimes \cdots \otimes p^{n}\right) \psi_{n} \otimes g^{n} \psi+\mu\right] .
$$

Moreover, if $\left(p^{1} \otimes \cdots \otimes p^{n}\right) \psi_{n}$ were a $\left(g^{0} \psi, g^{n} \psi\right)$-coderivation, it would imply (3.2.4) by Section 3.2. We deduce that, indeed, the above $\mu=0$, and $\left(p^{1} \otimes \cdots \otimes p^{n}\right) \psi_{n}$ is a $\left(g^{0} \psi, g^{n} \psi\right)$-coderivation. Thus, we have found a unique $\left(p^{1} \otimes \cdots \otimes p^{n}\right) \psi_{n} \in$ $\operatorname{Coder}(\mathcal{A}, \mathcal{B})$ and $(3.4 .1)$ for $m=n$ defines uniquely an element $\left(p^{1} \otimes \cdots \otimes p^{n}\right) \psi \in$ $T$ Coder $(\mathcal{A}, \mathcal{B})$.

The case $q>1$ is similar to the case $q=1$, however, the reasoning is slightly obstructed by a big amount of indices. So we explain in detail the case $q=2$ only, and in the general case no new phenomena occur. Further we shall use the obtained formulas in the case $q=2$.

Let $\mathcal{C}=\mathcal{C}^{1}, \mathcal{D}=\mathcal{C}^{2}$. We consider a cocategory homomorphism $\phi: T \mathcal{A} \otimes T \mathcal{C} \otimes$ $T \mathcal{D} \rightarrow T \mathcal{B}, a \mapsto a[(c \otimes d) \chi]$. We have to obtain from it a unique cocategory homomorphism $\psi: T \mathcal{C} \otimes T \mathcal{D} \rightarrow T \operatorname{Coder}(\mathcal{A}, \mathcal{B})$.

A pair of objects $f \in \mathrm{Ob} \mathcal{C}, g \in \mathrm{Ob} \mathcal{D}$ induces a cocategory morphism $(f, g) \psi$ : $a \mapsto\left(a \otimes 1_{f} \otimes 1_{g}\right) \phi$. We set $\psi_{00}=0$. An object $f \in \mathrm{Ob} \mathcal{C}$ and an element $t \in \mathcal{D}\left(g^{0}, g^{1}\right)$ induce an $\left(\left(f, g^{0}\right) \psi,\left(f, g^{1}\right) \psi\right)$-coderivation $(f \otimes t) \psi_{01}=(f \otimes t) \psi: a \mapsto\left(a \otimes 1_{f} \otimes t\right) \phi$. An element $p \in \mathcal{C}\left(f^{0}, f^{1}\right)$ and an object $g \in \mathrm{Ob} \mathcal{D}$ induce an $\left(\left(f^{0}, g\right) \psi,\left(f^{1}, g\right) \psi\right)$ coderivation $(p \otimes g) \psi_{10}=(p \otimes g) \psi: a \mapsto\left(a \otimes p \otimes 1_{g}\right) \phi$. Suppose that $\psi_{i j}$ are already found for $0 \leqslant i \leqslant n, 0 \leqslant j \leqslant m,(i, j) \neq(n, m)$. Then we find $\psi_{n m}$ from the sought identity $\chi=\psi \theta$. Namely, for $f^{0} \stackrel{p^{1}}{\longrightarrow} f^{1} \stackrel{p^{2}}{\longrightarrow} \ldots f^{n-1} \stackrel{p^{n}}{\longrightarrow} f^{n}$ in $\mathcal{C}$ and 
$g^{0} \stackrel{t^{1}}{\longrightarrow} g^{1} \stackrel{t^{2}}{\longrightarrow} \ldots g^{m-1} \stackrel{t^{m}}{\longrightarrow} g^{m}$ in $\mathcal{D}$ we have to satisfy the identity

$$
\begin{gathered}
\left(p^{1} \otimes \cdots \otimes p^{n} \otimes t^{1} \otimes \cdots \otimes t^{m}\right) \chi=\left(p^{1} \otimes \cdots \otimes p^{n} \otimes t^{1} \otimes \cdots \otimes t^{m}\right) \psi_{n m} \\
+\sum_{\substack{i_{1}+\cdots+i_{k}=n \\
j_{1}+\cdots+j_{k}=m}}^{k>1}(-)^{\sigma}\left[\left(p^{1} \otimes \cdots \otimes p^{i_{1}} \otimes t^{1} \otimes \cdots \otimes t^{j_{1}}\right) \psi_{i_{1} j_{1}}\right. \\
\left.\left.\otimes\left(p^{i_{1}+1} \otimes \cdots \otimes p^{i_{1}+i_{2}} \otimes t^{j_{1}+1} \otimes \cdots \otimes t^{j_{1}+j_{2}}\right) \psi_{i_{2} j_{2}} \otimes p^{i_{1}+\cdots+i_{k}} \otimes t^{j_{1}+\cdots+j_{k-1}+1} \otimes \cdots \otimes t^{j_{1}+\cdots+j_{k}}\right) \psi_{i_{k} j_{k}}\right] \theta
\end{gathered}
$$

which is nothing else but (3.2.1). The sign is determined by (3.2.2). All terms of the sum are already known. So we define a map $\left(p^{1} \otimes \cdots \otimes p^{n} \otimes t^{1} \otimes \cdots \otimes t^{m}\right) \psi_{n m}$ : $T \mathcal{A} \rightarrow T \mathcal{B}$ as the difference of the left hand side and the sum in the right hand side. The fact that $\phi$ is a homomorphism is equivalent to the identity for the map $\chi$ for all $n, m \geqslant 0$ :

$$
\begin{aligned}
& \left(p^{1} \otimes \cdots \otimes p^{n} \otimes t^{1} \otimes \cdots \otimes t^{m}\right) \chi \Delta=\Delta \sum_{k=0}^{n} \sum_{l=0}^{m}(-)^{\left(p^{k+1}+\cdots+p^{n}\right)\left(t^{1}+\cdots+t^{l}\right)} \\
& \quad\left(p^{1} \otimes \cdots \otimes p^{k} \otimes t^{1} \otimes \cdots \otimes t^{l}\right) \chi \otimes\left(p^{k+1} \otimes \cdots \otimes p^{n} \otimes t^{l+1} \otimes \cdots \otimes t^{m}\right) \chi,
\end{aligned}
$$

similarly to Section 3.2. From it we get an equation for $\left(p^{1} \otimes \cdots \otimes p^{n} \otimes t^{1} \otimes \cdots \otimes t^{m}\right) \psi_{n m}$

$$
\begin{array}{r}
\left(p^{1} \otimes \cdots \otimes p^{n} \otimes t^{1} \otimes \cdots \otimes t^{m}\right) \psi_{n m} \Delta=\Delta\left[\left(f^{0}, g^{0}\right) \psi \otimes\left(p^{1} \otimes \cdots \otimes p^{n} \otimes t^{1} \otimes \cdots \otimes t^{m}\right) \psi_{n m}\right. \\
\left.+\left(p^{1} \otimes \cdots \otimes p^{n} \otimes t^{1} \otimes \cdots \otimes t^{m}\right) \psi_{n m} \otimes\left(f^{n}, g^{m}\right) \psi+\mu\right] .
\end{array}
$$

Notice that if $\psi$ is indeed a homomorphism and $\psi_{n m}$ is its component, then $\phi$ is a homomorphism, hence, (3.4.3) holds. Thus, the above equation with $\mu=0$ implies (3.4.3) (and it happens only for one value of $\mu$ ). Since we know that (3.4.3) holds, it implies $\mu=0$. Therefore, $\left(p^{1} \otimes \cdots \otimes p^{n} \otimes t^{1} \otimes \cdots \otimes t^{m}\right) \psi_{n m}$ is a $\left(\left(f^{0}, g^{0}\right) \psi,\left(f^{n}, g^{m}\right) \psi\right)$ coderivation. Thus, we have found a unique $\left(p^{1} \otimes \cdots \otimes p^{n} \otimes t^{1} \otimes \cdots \otimes t^{m}\right) \psi_{n m} \in$ $\operatorname{Coder}(\mathcal{A}, \mathcal{B})$, and

$$
\begin{gathered}
\left(p^{1} \otimes \cdots \otimes p^{n} \otimes t^{1} \otimes \cdots \otimes t^{m}\right) \psi=\left(p^{1} \otimes \cdots \otimes p^{n} \otimes t^{1} \otimes \cdots \otimes t^{m}\right) \psi_{n m} \\
+\sum_{\substack{i_{1}+\cdots+i_{k}=n \\
j_{1}+\cdots+j_{k}=m}}(-)^{\sigma}\left(p^{1} \otimes \cdots \otimes p^{i_{1}} \otimes t^{1} \otimes \cdots \otimes t^{j_{1}}\right) \psi_{i_{1} j_{1}} \\
\otimes\left(p^{i_{1}+1} \otimes \cdots \otimes p^{i_{1}+i_{2}} \otimes t^{j_{1}+1} \otimes \cdots \otimes t^{j_{1}+j_{2}}\right) \psi_{i_{2} j_{2}} \\
\otimes \cdots \otimes\left(p^{i_{1}+\cdots+i_{k-1}+1} \otimes \cdots \otimes p^{i_{1}+\cdots+i_{k}} \otimes t^{j_{1}+\cdots+j_{k-1}+1} \otimes \cdots \otimes t^{j_{1}+\cdots+j_{k}}\right) \psi_{i_{k} j_{k}} \otimes \cdots
\end{gathered}
$$

defines uniquely an element of $T \operatorname{Coder}(\mathcal{A}, \mathcal{B})$. The above formula implies that $\psi$ is a homomorphism.

A generalization to $q>2$ is straightforward.

We interpret the above proposition as the existence of inner hom-objects $\operatorname{Hom}(T \mathcal{A}, T \mathcal{B})=T \operatorname{Coder}(\mathcal{A}, \mathcal{B})$ in the monoidal category of cocategories of the form $T \mathrm{C}^{1} \otimes T \mathrm{C}^{2} \otimes \cdots \otimes T \mathrm{C}^{r}$. 


\section{A category enriched in cocategories}

Let us show that the category of tensor coalgebras of graded $\mathbb{k}$-quivers is enriched in $\mathrm{gCoCat}$.

Let $\mathcal{A}, \mathcal{B}, \mathcal{C}$ be graded $\mathbb{k}$-quivers. Consider the cocategory homomorphism given by the upper right path in the diagram

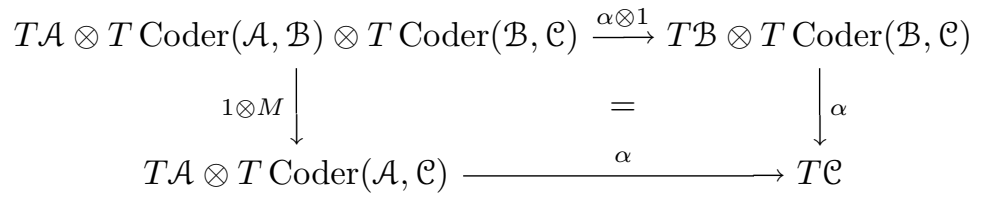

By Proposition 3.4 there is a graded cocategory morphism of degree 0

$$
M: T \operatorname{Coder}(\mathcal{A}, \mathcal{B}) \otimes T \operatorname{Coder}(\mathcal{B}, \mathcal{C}) \rightarrow T \operatorname{Coder}(\mathcal{A}, \mathcal{C}) .
$$

Denote by $\mathbb{1}$ a graded 1 -object-0-morphisms $\mathbb{k}$-quiver, that is, Ob $\mathbb{1}=\{*\}$, $\mathbb{1}(*, *)=0$. Then $T \mathbb{1}=\mathbb{k}$ is a unit object of the monoidal category of graded cocategories. Denote by $\mathbf{r}: T \mathcal{A} \otimes T \mathbb{1} \rightarrow T \mathcal{A}$ and $\mathbf{l}: T \mathbb{1} \otimes T \mathcal{A} \rightarrow T \mathcal{A}$ the corresponding natural cocategory isomorphisms. By Proposition 3.4 there exists a unique cocategory morphism $\eta_{\mathcal{A}}: T \mathbb{1} \rightarrow T \operatorname{Coder}(\mathcal{A}, \mathcal{A})$, such that

$$
\mathbf{r}=\left(T \mathcal{A} \otimes T \mathbb{1} \stackrel{1 \otimes \eta_{\mathcal{A}}}{\longrightarrow} T \mathcal{A} \otimes T \operatorname{Coder}(\mathcal{A}, \mathcal{A}) \stackrel{\alpha}{\longrightarrow} T \mathcal{A}\right) .
$$

Namely, the object $* \in \mathrm{Ob} \mathbb{1}$ goes to the identity homomorphism $\operatorname{id}_{\mathcal{A}}: \mathcal{A} \rightarrow \mathcal{A}$, which acts as the identity map on objects, and has only one non-vanishing component $\left(\operatorname{id}_{\mathcal{A}}\right)_{1}=\operatorname{id}: \mathcal{A}(X, Y) \rightarrow \mathcal{A}(X, Y)$.

4.1 Proposition (See also Kontsevich and Soibelman [KS]). The multiplication $M$ is associative and $\eta$ is its two-sided unit:

$T \operatorname{Coder}(\mathcal{A}, \mathcal{B}) \otimes T \operatorname{Coder}(\mathcal{B}, \mathcal{C}) \otimes T \operatorname{Coder}(\mathcal{C}, \mathcal{D}) \stackrel{M \otimes 1}{\longrightarrow} T \operatorname{Coder}(\mathcal{A}, \mathcal{C}) \otimes T \operatorname{Coder}(\mathcal{C}, \mathcal{D})$

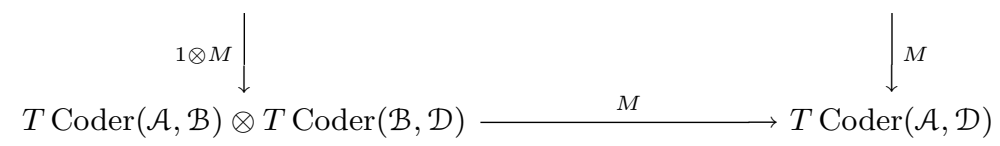

Proof. The cocategory homomorphism

$T \mathcal{A} \otimes T \operatorname{Coder}(\mathcal{A}, \mathcal{B}) \otimes T \operatorname{Coder}(\mathcal{B}, \mathcal{C}) \otimes T \operatorname{Coder}(\mathcal{C}, \mathcal{D})$

$\stackrel{\alpha \otimes 1 \otimes 1}{\longrightarrow} T \mathcal{B} \otimes T \operatorname{Coder}(\mathcal{B}, \mathcal{C}) \otimes T \operatorname{Coder}(\mathcal{C}, \mathcal{D}) \stackrel{\alpha \otimes 1}{\longrightarrow} T \mathcal{C} \otimes T \operatorname{Coder}(\mathcal{C}, \mathcal{D}) \stackrel{\alpha}{\longrightarrow} T \mathcal{D}$

can be written down as

$$
(\alpha \otimes 1 \otimes 1)(1 \otimes M) \alpha=(1 \otimes 1 \otimes M)(\alpha \otimes 1) \alpha=(1 \otimes 1 \otimes M)(1 \otimes M) \alpha,
$$

or as

$$
(1 \otimes M \otimes 1)(\alpha \otimes 1) \alpha=(1 \otimes M \otimes 1)(1 \otimes M) \alpha .
$$

The uniqueness part of Proposition 3.4 implies that $(1 \otimes M) M=(M \otimes 1) M$.

Similarly one proves that $\eta$ is a unit for $M$. 
By (2.4.1) we find

$\operatorname{gCoCat}(T \mathbb{1}, T \operatorname{Coder}(\mathcal{A}, \mathcal{B}))=\operatorname{Maps}(\{*\}, \operatorname{Ob} \operatorname{Coder}(\mathcal{A}, \mathcal{B}))=\operatorname{gCoCat}(T \mathcal{A}, T \mathcal{B})$.

Thus we can interpret Proposition 4.1 as saying that the category of tensor coalgebras of graded $\mathbb{k}$-quivers admits an enrichment in gCoCat.

Let us find explicit formulas for $M$. It is defined on objects as composition: if $f: \mathcal{A} \rightarrow \mathcal{B}$ and $g: \mathcal{B} \rightarrow \mathcal{C}$ are cocategory morphisms, then $(f, g) M=f g: \mathcal{A} \rightarrow \mathcal{C}$. On coderivations $M$ is specified by its composition with $\operatorname{pr}_{1}: T \operatorname{Coder}(\mathcal{A}, \mathcal{C}) \rightarrow$ $\operatorname{Coder}(\mathcal{A}, \mathcal{C})$. Let us write in this section $(h, k)$ as a shorthand for $\operatorname{Coder}(\mathcal{A}, \mathcal{C})(h, k)$, the $\mathbb{k}$-module of $(h, k)$-coderivations. The components of $M$ are

$$
\begin{gathered}
M_{n m}=\left.M\right|_{T^{n} \otimes T^{m}} \operatorname{pr}_{1}: T^{n} \operatorname{Coder}(\mathcal{A}, \mathcal{B}) \otimes T^{m} \operatorname{Coder}(\mathcal{B}, \mathcal{C}) \rightarrow \operatorname{Coder}(\mathcal{A}, \mathcal{C}), \\
M_{n m}:\left(f^{0}, f^{1}\right) \otimes \cdots \otimes\left(f^{n-1}, f^{n}\right) \otimes\left(g^{0}, g^{1}\right) \otimes \cdots \otimes\left(g^{m-1}, g^{m}\right) \rightarrow\left(f^{0} g^{0}, f^{n} g^{m}\right),
\end{gathered}
$$

where $f^{0}, \ldots, f^{n}: \mathcal{A} \rightarrow \mathcal{B}$ and $g^{0}, \ldots, g^{m}: \mathcal{B} \rightarrow \mathcal{C}$ are cocategory morphisms. We have $M_{00}=0$.

According to proof of Proposition 3.4 the component $M_{n m}$ is determined recursively from equation (3.4.2):

$$
\begin{gathered}
\left(p^{1} \otimes \cdots \otimes p^{n}\right) \theta\left(t^{1} \otimes \cdots \otimes t^{m}\right) \theta=\left(p^{1} \otimes \cdots \otimes p^{n} \otimes t^{1} \otimes \cdots \otimes t^{m}\right) M_{n m} \\
+\sum_{\substack{i_{1}+\cdots+i_{k}=n \\
j_{1}+\cdots+j_{k}=m}}^{k>1}(-)^{\sigma}\left[\left(p^{1} \otimes \cdots \otimes p^{i_{1}} \otimes t^{1} \otimes \cdots \otimes t^{j_{1}}\right) M_{i_{1} j_{1}}\right. \\
\otimes\left(p^{i_{1}+1} \otimes \cdots \otimes p^{i_{1}+i_{2}} \otimes t^{j_{1}+1} \otimes \cdots \otimes t^{j_{1}+j_{2}}\right) M_{i_{2} j_{2}} \\
\left.\otimes \cdots \otimes\left(p^{i_{1}+\cdots+i_{k-1}+1} \otimes \cdots \otimes p^{i_{1}+\cdots+i_{k}} \otimes t^{j_{1}+\cdots+j_{k-1}+1} \otimes \cdots \otimes t^{j_{1}+\cdots+j_{k}}\right) M_{i_{k} j_{k}}\right] \theta .
\end{gathered}
$$

Since $\left(p^{1} \otimes \cdots \otimes p^{n} \otimes t^{1} \otimes \cdots \otimes t^{m}\right) M_{n m}$ is a coderivation, it is determined by its composition with projection $\mathrm{pr}_{1}$. Composing (4.1.1) with $\mathrm{pr}_{1}$ we get

$$
\left(p^{1} \otimes \cdots \otimes p^{n}\right) \theta\left(t^{1} \otimes \cdots \otimes t^{m}\right) \theta \mathrm{pr}_{1}=\left(p^{1} \otimes \cdots \otimes p^{n} \otimes t^{1} \otimes \cdots \otimes t^{m}\right) M_{n m} \mathrm{pr}_{1} .
$$

Therefore, if $m=0$ and $n$ is positive, $M_{n 0}$ is given by the formula:

$$
\begin{aligned}
& M_{n 0}:\left(f^{0}, f^{1}\right) \otimes \cdots \otimes\left(f^{n-1}, f^{n}\right) \otimes \mathbb{k}_{g^{0}} \\
& r^{1} \otimes \cdots \otimes\left(f^{0} g^{0}, f^{n} g^{0}\right), \\
& {\left[\left(r^{1} \otimes \cdots \otimes r^{n} \mid g^{0}\right) M_{n 0}\right] \operatorname{pr}_{1}=\left(r^{1} \otimes \cdots \otimes r^{n}\right) \theta g^{0} \mathrm{pr}_{1}, }
\end{aligned}
$$

where $\mid$ separates the arguments in place of $\otimes$. If $m=1$, then $M_{n 1}$ is given by the formula:

$$
\begin{gathered}
M_{n 1}:\left(f^{0}, f^{1}\right) \otimes \cdots \otimes\left(f^{n-1}, f^{n}\right) \otimes\left(g^{0}, g^{1}\right) \rightarrow\left(f^{0} g^{0}, f^{n} g^{1}\right), \\
r^{1} \otimes \cdots \otimes r^{n} \otimes t^{1} \mapsto\left(r^{1} \otimes \cdots \otimes r^{n} \otimes t^{1}\right) M_{n 1}, \\
{\left[\left(r^{1} \otimes \cdots \otimes r^{n} \otimes t^{1}\right) M_{n 1}\right] \mathrm{pr}_{1}=\left(r^{1} \otimes \cdots \otimes r^{n}\right) \theta t^{1} \mathrm{pr}_{1} .}
\end{gathered}
$$


Explicitly we write

$$
\begin{aligned}
& {\left[\left(r^{1} \otimes \cdots \otimes r^{n} \mid g^{0}\right) M_{n 0}\right]_{k}=\sum_{l}\left(r^{1} \otimes \cdots \otimes r^{n}\right) \theta_{k l} g_{l}^{0},} \\
& {\left[\left(r^{1} \otimes \cdots \otimes r^{n} \otimes t^{1}\right) M_{n 1}\right]_{k}=\sum_{l}\left(r^{1} \otimes \cdots \otimes r^{n}\right) \theta_{k l} t_{l}^{1} .}
\end{aligned}
$$

Finally, $M_{n m}=0$ for $m>1$, since the left hand side of (4.1.2) vanishes.

4.2 Examples. 1) The component $M_{01}$ is the composition: $\left(f^{0} \mid t^{1}\right) M_{01}=f^{0} t^{1}$.

2) The component $M_{10}$ is the composition: $\left(r^{1} \mid g^{0}\right) M_{10}=r^{1} g^{0}$.

3) If $r: f \rightarrow g: \mathcal{A} \rightarrow \mathcal{B}$ and $p: h \rightarrow k: \mathcal{B} \rightarrow \mathcal{C}$ are $A_{\infty}$-transformations, then $(r \otimes p) M_{11}: f h \rightarrow g k: \mathcal{A} \rightarrow \mathcal{C}$ has the following components:

$$
\begin{aligned}
& {\left[(r \otimes p) M_{11}\right]_{0}=r_{0} p_{1},} \\
& {\left[(r \otimes p) M_{11}\right]_{1}=r_{1} p_{1}+\left(f_{1} \otimes r_{0}\right) p_{2}+\left(r_{0} \otimes g_{1}\right) p_{2}, \text { etc. }}
\end{aligned}
$$

4) If $f \stackrel{r}{\longrightarrow} g \stackrel{p}{\longrightarrow} h: \mathcal{A} \rightarrow \mathcal{B}$ are $A_{\infty}$-transformations, and $k: \mathcal{B} \rightarrow \mathcal{C}$ is an $A_{\infty}$-functor, then $(r \otimes p \mid k) M_{20}: f k \rightarrow h k: \mathcal{A} \rightarrow \mathcal{C}$ has the following components:

$$
\begin{aligned}
{\left[(r \otimes p \mid k) M_{20}\right]_{0}=} & \left(r_{0} \otimes p_{0}\right) k_{2}, \\
{\left[(r \otimes p \mid k) M_{20}\right]_{1}=} & \left(r_{1} \otimes p_{0}\right) k_{2}+\left(r_{0} \otimes p_{1}\right) k_{2} \\
& +\left(r_{0} \otimes p_{0} \otimes h_{1}\right) k_{3}+\left(r_{0} \otimes g_{1} \otimes p_{0}\right) k_{3}+\left(f_{1} \otimes r_{0} \otimes p_{0}\right) k_{3}, \text { etc. }
\end{aligned}
$$

5) If $f \stackrel{r}{\longrightarrow} g \stackrel{p}{\longrightarrow} h: \mathcal{A} \rightarrow \mathcal{B}$ and $t: k \rightarrow l: \mathcal{B} \rightarrow \mathcal{C}$ are $A_{\infty}$-transformations, then $(r \otimes p \otimes t) M_{21}: f k \rightarrow h l: \mathcal{A} \rightarrow \mathcal{C}$ has the following components:

$$
\begin{aligned}
{\left[(r \otimes p \otimes t) M_{21}\right]_{0}=} & \left(r_{0} \otimes p_{0}\right) t_{2}, \\
{\left[(r \otimes p \otimes t) M_{21}\right]_{1}=} & \left(r_{1} \otimes p_{0}\right) t_{2}+\left(r_{0} \otimes p_{1}\right) t_{2} \\
& +\left(r_{0} \otimes p_{0} \otimes h_{1}\right) t_{3}+\left(r_{0} \otimes g_{1} \otimes p_{0}\right) t_{3}+\left(f_{1} \otimes r_{0} \otimes p_{0}\right) t_{3}, \text { etc. }
\end{aligned}
$$

\section{5. $A_{\infty}$-category of $A_{\infty}$-functors}

Let us construct a new $A_{\infty}$-category $A_{\infty}(\mathcal{A}, \mathcal{B})$ out of given two $\mathcal{A}$ and $\mathcal{B}$. Its underlying graded $\mathbb{k}$-quiver is a full subquiver of $s^{-1} \operatorname{Coder}(s \mathcal{A}, s \mathcal{B})$. The objects of $A_{\infty}(\mathcal{A}, \mathcal{B})$ are $A_{\infty}$-functors $f: \mathcal{A} \rightarrow \mathcal{B}$. Given two such functors $f, g: \mathcal{A} \rightarrow \mathcal{B}$ we define the graded $\mathbb{k}$-module $A_{\infty}(\mathcal{A}, \mathcal{B})(f, g)$ as the space of all $A_{\infty}$-transformations $r: f \rightarrow g$, namely,

$$
\begin{gathered}
{\left[A_{\infty}(\mathcal{A}, \mathcal{B})(f, g)\right]^{d+1}=} \\
\left\{r: f \rightarrow g \mid A_{\infty} \text {-transformation } r: T s \mathcal{A} \rightarrow T s \mathcal{B} \text { has degree } d\right\} .
\end{gathered}
$$

In this section we use the notation $(f, g)=s A_{\infty}(\mathcal{A}, \mathcal{B})(f, g)=\operatorname{Coder}(s \mathcal{A}, s \mathcal{B})(f, g)$ for the sake of brevity. The degree of $r$ as an element of $(f, g)$ will be exactly $d$ :

$$
(f, g)^{d}=\left\{r: f \rightarrow g \mid A_{\infty} \text {-transformation } r: T s \mathcal{A} \rightarrow T s \mathcal{B} \text { has degree } d\right\} .
$$

We will use only this (natural) degree of $r$ in order to permute it with other things by Koszul's rule. 
Notice that even if $\mathcal{A}, \mathcal{B}$ have one object (and are $A_{\infty}$-algebras), the quiver $A_{\infty}(\mathcal{A}, \mathcal{B})$ has several objects. Thus theory of $A_{\infty}$-algebras leads to the theory of $A_{\infty}$-categories.

5.1 Proposition (See also Fukaya [Fuk], Kontsevich and Soibelman [KS02, KS] and Lefèvre-Hasegawa [LH02]). Let $\mathcal{A}, \mathcal{B}$ be $A_{\infty}$-categories. Then there exists a unique $(1,1)$-coderivation $B: T s A_{\infty}(\mathcal{A}, \mathcal{B}) \rightarrow T s A_{\infty}(\mathcal{A}, \mathcal{B})$ of degree 1 , such that $B_{0}=0$ and

$$
\left(r^{1} \otimes \cdots \otimes r^{n}\right) \theta b=\left[\left(r^{1} \otimes \cdots \otimes r^{n}\right) B\right] \theta+(-)^{r^{1}+\cdots+r^{n}} b\left(r^{1} \otimes \cdots \otimes r^{n}\right) \theta
$$

for all $n \geqslant 0, r^{1} \otimes \cdots \otimes r^{n} \in\left(f^{0}, f^{1}\right) \otimes \cdots \otimes\left(f^{n-1}, f^{n}\right)$. It satisfies $B^{2}=0$, thus, it gives an $A_{\infty}$-structure of $A_{\infty}(\mathcal{A}, \mathcal{B})$.

Proof. For $n=0$ (5.1.1) reads as $f^{0} b=\left(f^{0}\right) B+b f^{0}$, hence, $\left(f^{0}\right) B=f^{0} b-b f^{0}=0$. In particular, we may set $B_{0}=0$. Assume that the coderivation components $B_{j}$ for $j<n$ are already found, so that (5.1.1) is satisfied up to $n-1$ arguments. Let us determine a $\mathbb{k}$-linear map $\left(r^{1} \otimes \cdots \otimes r^{n}\right) B_{n}: T s \mathcal{A} \rightarrow T s \mathcal{B}$ from equation (5.1.1), rewritten as follows:

$$
\begin{aligned}
\left(r^{1} \otimes \cdots \otimes r^{n}\right) B_{n}=\left(r^{1} \otimes\right. & \left.\cdots \otimes r^{n}\right) \theta b-(-)^{r^{1}+\cdots+r^{n}} b\left(r^{1} \otimes \cdots \otimes r^{n}\right) \theta \\
& -\sum_{q+j+t=n}^{j<n}\left[\left(r^{1} \otimes \cdots \otimes r^{n}\right)\left(1^{\otimes q} \otimes B_{j} \otimes 1^{\otimes t}\right)\right] \theta .
\end{aligned}
$$

Let us show that $\left(r^{1} \otimes \cdots \otimes r^{n}\right) B_{n}$ is a $\left(f^{0}, f^{n}\right)$-coderivation. Indeed,

$$
\begin{aligned}
& \left(r^{1} \otimes \cdots \otimes r^{n}\right) B_{n} \Delta_{\mathcal{B}}=\left(r^{1} \otimes \cdots \otimes r^{n}\right) \theta b \Delta_{\mathcal{B}}-(-)^{r^{1}+\cdots+r^{n}} b\left(r^{1} \otimes \cdots \otimes r^{n}\right) \theta \Delta_{\mathcal{B}} \\
& -\sum_{q+j+t=n}^{j<n}\left[\left(r^{1} \otimes \cdots \otimes r^{n}\right)\left(1^{\otimes q} \otimes B_{j} \otimes 1^{\otimes t}\right)\right] \theta \Delta_{\mathcal{B}} \\
& =\left(r^{1} \otimes \cdots \otimes r^{n}\right) \theta \Delta_{\mathcal{B}}(1 \otimes b+b \otimes 1) \\
& -(-)^{r^{1}+\cdots+r^{n}} b \Delta_{\mathcal{A}} \sum_{k=0}^{n}\left(r^{1} \otimes \cdots \otimes r^{k}\right) \theta \otimes\left(r^{k+1} \otimes \cdots \otimes r^{n}\right) \theta \\
& -\sum_{q+j+t=n}^{j<n} \Delta_{\mathcal{A}}\left\{\left[\left(r^{1} \otimes \cdots \otimes r^{n}\right)\left(1^{\otimes q} \otimes B_{j} \otimes 1^{\otimes t}\right)\right] \Delta_{A_{\infty}(\mathcal{A}, \mathcal{B})}(\theta \otimes \theta)\right\}
\end{aligned}
$$




$$
\begin{aligned}
=\Delta_{\mathcal{A}} & \left\{\sum_{k=0}^{n}\left(r^{1} \otimes \cdots \otimes r^{k}\right) \theta \otimes\left(r^{k+1} \otimes \cdots \otimes r^{n}\right) \theta(1 \otimes b+b \otimes 1)\right. \\
& -(-)^{r^{1}+\cdots+r^{n}}(1 \otimes b+b \otimes 1) \sum_{k=0}^{n}\left(r^{1} \otimes \cdots \otimes r^{k}\right) \theta \otimes\left(r^{k+1} \otimes \cdots \otimes r^{n}\right) \theta \\
& -\sum_{k+v+j+t=n}^{j<n}\left[\left(r^{1} \otimes \cdots \otimes r^{n}\right)\left(1^{\otimes k} \otimes 1^{\otimes v} \otimes B_{j} \otimes 1^{\otimes t}\right)\right](\theta \otimes \theta) \\
& \left.-\sum_{q+j+w+u=n}^{j<n}\left[\left(r^{1} \otimes \cdots \otimes r^{n}\right)\left(1^{\otimes q} \otimes B_{j} \otimes 1^{\otimes w} \otimes 1^{\otimes u}\right)\right](\theta \bigotimes \theta)\right\} \\
=\Delta_{\mathcal{A}} & \left\{\sum_{k=0}^{n}\left(r^{1} \otimes \cdots \otimes r^{k}\right) \theta \otimes\left(r^{k+1} \otimes \cdots \otimes r^{n}\right) \theta b\right. \\
- & \sum_{k=0}^{n}(-)^{r^{k+1}+\cdots+r^{n}}\left(r^{1} \otimes \cdots \otimes r^{k}\right) \theta \otimes b\left(r^{k+1} \otimes \cdots \otimes r^{n}\right) \theta \\
- & \sum_{k+v+j+t=n}^{j<n}\left(r^{1} \otimes \cdots \otimes r^{k}\right) \theta \otimes\left(r^{k+1} \otimes \cdots \otimes r^{n}\right)\left(1^{\otimes v} \otimes B_{j} \otimes 1^{\otimes t}\right) \theta \\
& +\sum_{k=0}^{n}(-)^{r^{k+1}+\cdots+r^{n}}\left(r^{1} \otimes \cdots \otimes r^{k}\right) \theta b \otimes\left(r^{k+1} \otimes \cdots \otimes r^{n}\right) \theta \\
& -\sum_{k=0}^{n}(-)^{r^{1}+\cdots+r^{n}} b\left(r^{1} \otimes \cdots \otimes r^{k}\right) \theta \otimes\left(r^{k+1} \otimes \cdots \otimes r^{n}\right) \theta \\
& -\sum_{k=0}^{n}(-)^{r^{k+1}+\cdots+r^{n}} \sum_{q}^{j<n}\left(f^{0} \otimes\left(r^{1} \otimes \cdots \otimes r^{n}\right) B_{n}+\left(r^{1} \otimes \cdots \otimes r^{n}\right) B_{n} \otimes f^{n}\right] . \\
\otimes & \left.\left(r^{k+1} \otimes \cdots \otimes r^{n}\right) \theta\right\} \\
= &
\end{aligned}
$$

The last three sums cancel out for all $k<n$ due to (5.1.2), and for $k=n$ they give $\left(r^{1} \otimes \cdots \otimes r^{n}\right) B_{n} \otimes f^{n}$ due to the same equation. Similarly for the previous three sums. Therefore, (5.1.2) is, indeed, a recursive definition of components $B_{n}$ of a coderivation $B$. The uniqueness of $B$ is obvious.

Clearly, $B^{2}: T s A_{\infty}(\mathcal{A}, \mathcal{B}) \rightarrow T s A_{\infty}(\mathcal{A}, \mathcal{B})$ is a $(1,1)$-coderivation of degree 2 . From (5.1.1) we find

$$
\begin{gathered}
{\left[\left(r^{1} \otimes \cdots \otimes r^{n}\right) B^{2}\right] \theta=\left[\left(r^{1} \otimes \cdots \otimes r^{n}\right) B\right] \theta b-(-)^{r^{1}+\cdots+r^{n}+1} b\left[\left(r^{1} \otimes \cdots \otimes r^{n}\right) B\right] \theta} \\
=\left(r^{1} \otimes \cdots \otimes r^{n}\right) \theta b^{2}-(-)^{r^{1}+\cdots+r^{n}} b\left[\left(r^{1} \otimes \cdots \otimes r^{n}\right) \theta\right] b \\
-(-)^{r^{1}+\cdots+r^{n}+1} b\left[\left(r^{1} \otimes \cdots \otimes r^{n}\right) \theta\right] b-b^{2}\left(r^{1} \otimes \cdots \otimes r^{n}\right) \theta=0 .
\end{gathered}
$$

Composing this equation with $\operatorname{pr}_{1}: T s \mathcal{B} \rightarrow s \mathcal{B}$ we get

$$
0=\left[\left(r^{1} \otimes \cdots \otimes r^{n}\right) B^{2}\right] \theta \mathrm{pr}_{1}=\left(r^{1} \otimes \cdots \otimes r^{n}\right)\left[B^{2}\right]_{n} \mathrm{pr}_{1} .
$$


Therefore, all components of the $\left(f^{0}, f^{n}\right)$-coderivation $\left(r^{1} \otimes \cdots \otimes r^{n}\right)\left[B^{2}\right]_{n}$ vanish. We deduce that the coderivations $\left(r^{1} \otimes \cdots \otimes r^{n}\right)\left[B^{2}\right]_{n}$ vanish, hence, all $\left[B^{2}\right]_{n}=0$. Finally, $B^{2}=0$.

Let us find explicitly the components of $B$, composing (5.1.1) with $\operatorname{pr}_{1}: T s \mathcal{B} \rightarrow$ $s \mathcal{B}$ :

$$
\begin{aligned}
B_{1}:(f, g) \rightarrow(f, g), \quad & r \mapsto(r) B_{1}=[r, b]=r b-(-)^{r} b r, \\
B_{n}:\left(f^{0}, f^{1}\right) \otimes \cdots \otimes\left(f^{n-1}, f^{n}\right) \rightarrow & \left(f^{0}, f^{n}\right), r^{1} \otimes \cdots \otimes r^{n} \mapsto\left(r^{1} \otimes \cdots \otimes r^{n}\right) B_{n}, \\
& \text { for } n>1,
\end{aligned}
$$

where the last transformation is defined by its composition with $\operatorname{pr}_{1}$ :

$$
\left[\left(r^{1} \otimes \cdots \otimes r^{n}\right) B_{n}\right] \mathrm{pr}_{1}=\left[\left(r^{1} \otimes \cdots \otimes r^{n}\right) \theta\right] b \mathrm{pr}_{1}
$$

In the other terms, for $n>1$

$$
\left[\left(r^{1} \otimes \cdots \otimes r^{n}\right) B_{n}\right]_{k}=\sum_{l}\left(r^{1} \otimes \cdots \otimes r^{n}\right) \theta_{k l} b_{l}
$$

Since $B^{2}=0$, we have, in particular,

$$
\sum_{r+n+t=k}\left(1^{\otimes r} \otimes B_{n} \otimes 1^{\otimes t}\right) B_{r+1+t}=0: T^{k} s A_{\infty}(\mathcal{A}, \mathcal{B}) \rightarrow s A_{\infty}(\mathcal{A}, \mathcal{B}) .
$$

5.2 Examples. 1) When $n=1, r: f \rightarrow g: \mathcal{A} \rightarrow \mathcal{B}$, we find the components of the $A_{\infty}$-transformation $(r) B_{1}: f \rightarrow g: \mathcal{A} \rightarrow \mathcal{B}$ as follows (see Examples 2.7):

$$
\begin{aligned}
{\left[(r) B_{1}\right]_{0} } & =r_{0} b_{1} \\
{\left[(r) B_{1}\right]_{1} } & =r_{1} b_{1}+\left(f_{1} \otimes r_{0}\right) b_{2}+\left(r_{0} \otimes g_{1}\right) b_{2}-(-)^{r} b_{1} r_{1} \\
{\left[(r) B_{1}\right]_{2} } & =r_{2} b_{1}+\left(f_{2} \otimes r_{0}\right) b_{2}+\left(f_{1} \otimes r_{1}\right) b_{2}+\left(r_{1} \otimes g_{1}\right) b_{2}+\left(r_{0} \otimes g_{2}\right) b_{2} \\
& +\left(f_{1} \otimes f_{1} \otimes r_{0}\right) b_{3}+\left(f_{1} \otimes r_{0} \otimes g_{1}\right) b_{3}+\left(r_{0} \otimes g_{1} \otimes g_{1}\right) b_{3}-(-)^{r} b_{2} r_{1} \\
& -(-)^{r}\left(1 \otimes b_{1}\right) r_{2}-(-)^{r}\left(b_{1} \otimes 1\right) r_{2} .
\end{aligned}
$$

2) When $n=2, f \stackrel{r}{\longrightarrow} g \stackrel{p}{\longrightarrow} h: \mathcal{A} \rightarrow \mathcal{B}$, we find the components of the $A_{\infty}$-transformation $(r \otimes p) B_{2}: f \rightarrow h: \mathcal{A} \rightarrow \mathcal{B}$ as follows:

$$
\begin{aligned}
{\left[(r \otimes p) B_{2}\right]_{0}=} & \left(r_{0} \otimes p_{0}\right) b_{2}, \\
{\left[(r \otimes p) B_{2}\right]_{1}=} & \left(r_{1} \otimes p_{0}\right) b_{2}+\left(r_{0} \otimes p_{1}\right) b_{2} \\
& +\left(r_{0} \otimes p_{0} \otimes h_{1}\right) b_{3}+\left(r_{0} \otimes g_{1} \otimes p_{0}\right) b_{3}+\left(f_{1} \otimes r_{0} \otimes p_{0}\right) b_{3}, \\
{\left[(r \otimes p) B_{2}\right]_{2}=} & \left(r_{2} \otimes p_{0}\right) b_{2}+\left(r_{1} \otimes p_{1}\right) b_{2}+\left(r_{0} \otimes p_{2}\right) b_{2}+\left(r_{1} \otimes p_{0} \otimes h_{1}\right) b_{3} \\
& +\left(r_{0} \otimes p_{1} \otimes h_{1}\right) b_{3}+\left(r_{1} \otimes g_{1} \otimes p_{0}\right) b_{3}+\left(r_{0} \otimes g_{1} \otimes p_{1}\right) b_{3} \\
& +\left(f_{1} \otimes r_{1} \otimes p_{0}\right) b_{3}+\left(f_{1} \otimes r_{0} \otimes p_{1}\right) b_{3}+\left(r_{0} \otimes p_{0} \otimes h_{2}\right) b_{3} \\
& +\left(r_{0} \otimes g_{2} \otimes p_{0}\right) b_{3}+\left(f_{2} \otimes r_{0} \otimes p_{0}\right) b_{3}+\left(r_{0} \otimes p_{0} \otimes h_{1} \otimes h_{1}\right) b_{4} \\
& +\left(r_{0} \otimes g_{1} \otimes g_{1} \otimes p_{0}\right) b_{4}+\left(r_{0} \otimes g_{1} \otimes p_{0} \otimes h_{1}\right) b_{4} \\
& +\left(f_{1} \otimes r_{0} \otimes p_{0} \otimes h_{1}\right) b_{4}+\left(f_{1} \otimes r_{0} \otimes g_{1} \otimes p_{0}\right) b_{4} \\
& +\left(f_{1} \otimes f_{1} \otimes r_{0} \otimes p_{0}\right) b_{4} .
\end{aligned}
$$


3) When $n=3, f \stackrel{r}{\longrightarrow} g \stackrel{p}{\longrightarrow} h \stackrel{t}{\longrightarrow} k: \mathcal{A} \rightarrow \mathcal{B}$, we find the components of the $A_{\infty}$-transformation $(r \otimes p \otimes t) B_{3}: f \rightarrow k: \mathcal{A} \rightarrow \mathcal{B}$ as follows:

$$
\begin{aligned}
{\left[(r \otimes p \otimes t) B_{3}\right]_{0}=} & \left(r_{0} \otimes p_{0} \otimes t_{0}\right) b_{3}, \\
{\left[(r \otimes p \otimes t) B_{3}\right]_{1}=} & \left(r_{1} \otimes p_{0} \otimes t_{0}\right) b_{3}+\left(r_{0} \otimes p_{1} \otimes t_{0}\right) b_{3}+\left(r_{0} \otimes p_{0} \otimes t_{1}\right) b_{3} \\
& +\left(r_{0} \otimes p_{0} \otimes t_{0} \otimes k_{1}\right) b_{4}+\left(r_{0} \otimes p_{0} \otimes h_{1} \otimes t_{0}\right) b_{4} \\
& +\left(r_{0} \otimes g_{1} \otimes p_{0} \otimes t_{0}\right) b_{4}+\left(f_{1} \otimes r_{0} \otimes p_{0} \otimes t_{0}\right) b_{4} .
\end{aligned}
$$

5.3. Differentials. Let $\mathcal{A}, \mathfrak{e}^{1}, \mathfrak{C}^{2}, \ldots, \mathfrak{e}^{q}, \mathcal{B}$ be $A_{\infty}$-categories. Let $\phi: T s \mathcal{A} \otimes$ $T s \mathrm{C}^{1} \otimes T s \mathrm{C}^{2} \otimes \cdots \otimes T s \mathrm{C}^{q} \rightarrow T s \mathcal{B},\left(a \otimes c^{1} \otimes c^{2} \otimes \cdots \otimes c^{q}\right) \mapsto a .\left(c^{1} \otimes c^{2} \otimes \cdots \otimes c^{q}\right) \chi$ be a cocategory homomorphism of degree 0 . If the homomorphism $\phi$ commutes with the differential:

$$
\phi b=\left(\sum_{r+t=q} 1^{\otimes r} \otimes b \otimes 1^{\otimes t}\right) \phi,
$$

then $\phi$ is called an $A_{\infty}$-functor (in a generalized sense, extending Definition 2.4). This condition is fulfilled if and only if $\chi$ commutes with the differential:

$$
\begin{aligned}
& \left(c^{1} \otimes c^{2} \otimes \cdots \otimes c^{q}\right) \chi b \\
= & \sum_{k=1}^{q}(-)^{c^{k+1}+\cdots+c^{q}}\left(c^{1} \otimes \cdots \otimes c^{k} b \otimes \cdots \otimes c^{q}\right) \chi+(-)^{c^{1}+\cdots+c^{q}} b\left(c^{1} \otimes c^{2} \otimes \cdots \otimes c^{q}\right) \chi .
\end{aligned}
$$

In particular, for $q=1$ we get the equation

$$
\text { (c) } \chi b=(c b) \chi+(-)^{c} b(c) \chi .
$$

5.4 Corollary (to Proposition 5.1). There is a unique $A_{\infty}$-category structure for $A_{\infty}(\mathcal{A}, \mathcal{B})$, such that the action homomorphism $\alpha: T s \mathcal{A} \otimes T s A_{\infty}(\mathcal{A}, \mathcal{B}) \rightarrow T s \mathcal{B}$ is an $A_{\infty}$-functor.

Proof. The homomorphism $\alpha: a \otimes r^{1} \otimes \cdots \otimes r^{n} \mapsto a\left[\left(r^{1} \otimes \cdots \otimes r^{n}\right) \theta\right]$ of Corollary 3.3 uses $\chi=\theta$. Hence, $\alpha$ is an $A_{\infty}$-functor if and only if $(r) \theta b=(r B) \theta+(-)^{r} b(r) \theta$ for $r=r^{1} \otimes \cdots \otimes r^{n}, n \geqslant 0$, that is, if and only if equations (5.1.1) hold.

5.5 Proposition. For any $A_{\infty}$-functor $\phi: T s \mathcal{A} \otimes T s \mathrm{C}^{1} \otimes T s \mathrm{C}^{2} \otimes \cdots \otimes T s \mathrm{C}^{q} \rightarrow T s \mathcal{B}$ there is a unique $A_{\infty}$-functor $\psi: T s \mathrm{C}^{1} \otimes T s \mathrm{C}^{2} \otimes \cdots \otimes T s \mathrm{C}^{q} \rightarrow T s A_{\infty}(\mathcal{A}, \mathcal{B})$, such that

$$
\phi=\left(T s \mathcal{A} \otimes T s \mathcal{C}^{1} \otimes T s \mathcal{C}^{2} \otimes \cdots \otimes T s \mathcal{C}^{q} \stackrel{1 \otimes \psi}{\longrightarrow} T s \mathcal{A} \otimes T s A_{\infty}(\mathcal{A}, \mathcal{B}) \stackrel{\alpha}{\longrightarrow} T s \mathcal{B}\right) .
$$

Proof. If $f^{i} \in \mathrm{ObC}^{i}$, then (5.3.1) implies that the cocategory homomorphism $\left(f^{1}, f^{2}, \ldots, f^{q}\right) \psi=\left(f^{1}, f^{2}, \ldots, f^{q}\right) \chi$ of degree 0 commutes with the differential $b$. Hence, it is an $A_{\infty}$-functor, that is, an object of $A_{\infty}(\mathcal{A}, \mathcal{B})$. By Proposition 3.4 there exists a unique cocategory homomorphism $\psi: T s \mathrm{C}^{1} \otimes T s \mathrm{C}^{2} \otimes \cdots \otimes T s \mathrm{C}^{q} \rightarrow$ $T s A_{\infty}(\mathcal{A}, \mathcal{B})$, of degree 0 , such that (5.5.1) holds. We have to prove that $\psi$ commutes with the differential. 
Using (5.1.1) and (5.3.1) we find for $\chi=\psi \theta$ the equation

$$
\begin{aligned}
\left(c^{1} \otimes c^{2} \otimes \cdots \otimes c^{q}\right) \psi B \theta & =\left(c^{1} \otimes c^{2} \otimes \cdots \otimes c^{q}\right) \psi \theta b-(-)^{c^{1}+\cdots+c^{q}} b\left(c^{1} \otimes c^{2} \otimes \cdots \otimes c^{q}\right) \psi \theta \\
& =\left(c^{1} \otimes c^{2} \otimes \cdots \otimes c^{q}\right) \sum_{k=1}^{q}\left(1^{\otimes k-1} \otimes b \otimes 1^{\otimes q-k}\right) \psi \theta . \quad \text { (5.5.2) }
\end{aligned}
$$

Notice that $\psi B$ and $\kappa \stackrel{\text { def }}{=} \sum_{k=1}^{q}\left(1^{\otimes k-1} \otimes b \otimes 1^{\otimes q-k}\right) \psi$ are both $(\psi, \psi)$-coderivations. Let $c^{i}$ be an element of $T^{n^{i}} s \mathcal{C}^{i}=s \mathcal{C}^{i}\left(f^{i},-\right) \otimes \cdots \otimes s \mathcal{C}^{i}\left(-, g^{i}\right)$ for $1 \leqslant i \leqslant q$. Composing (5.5.2) with $\operatorname{pr}_{1}: T s \mathcal{B} \rightarrow s \mathcal{B}$ we get

$$
\begin{gathered}
\left(c^{1} \otimes c^{2} \otimes \cdots \otimes c^{q}\right)[\psi B]_{n^{1} \ldots n^{q}} \operatorname{pr}_{1}=\left(c^{1} \otimes c^{2} \otimes \cdots \otimes c^{q}\right) \psi B \theta \mathrm{pr}_{1} \\
=\left(c^{1} \otimes c^{2} \otimes \cdots \otimes c^{q}\right) \kappa \theta \mathrm{pr}_{1}=\left(c^{1} \otimes c^{2} \otimes \cdots \otimes c^{q}\right) \kappa_{n^{1} \ldots n^{q}} \mathrm{pr}_{1} .
\end{gathered}
$$

Since all components of $\left(\left(f^{1}, f^{2}, \ldots, f^{q}\right) \psi,\left(g^{1}, g^{2}, \ldots, g^{q}\right) \psi\right)$-coderivations $\left(c^{1} \otimes c^{2} \otimes\right.$ $\left.\cdots \otimes c^{q}\right)[\psi B]_{n^{1} \ldots n^{q}}$ and $\left(c^{1} \otimes c^{2} \otimes \cdots \otimes c^{q}\right) \kappa_{n^{1} \ldots n^{q}}$ coincide, these coderivations coincide as well. Therefore, all the components of $(\psi, \psi)$-coderivations $\psi B$ and $\kappa$ coincide. We conclude that these coderivations coincide as well: $\psi B=\kappa=\sum_{k=1}^{q}\left(1^{\otimes k-1} \otimes\right.$ $\left.b \otimes 1^{\otimes q-k}\right) \psi$.

\section{Enriched category of $A_{\infty}$-categories}

6.1 Definition (Differential graded cocategory). A differential graded cocategory $\mathcal{C}$ is a graded cocategory equipped with a $(1,1)$-coderivation $b=(b: \mathcal{C}(X, Y) \rightarrow$ $\mathcal{C}(X, Y))_{X, Y \in \text { Ob e }}$ of degree 1 , such that $b^{2}=0$.

As in Section 2.2 a differential graded cocategory can be identified with a differential graded $\mathbb{k}$-coalgebra, decomposed in a special way. An example of a differential graded cocategory is given by $T s \mathcal{A}$, where $\mathcal{A}$ is an $A_{\infty}$-category.

Differential graded cocategories form a symmetric monoidal category dgCoCat. If $\mathcal{C}$ and $\mathcal{D}$ are differential graded cocategories then their tensor product is the graded cocategory $\mathcal{C} \otimes \mathcal{D}$, equipped with the differential $1 \otimes b+b \otimes 1$. We want to show that the category of $A_{\infty}$-categories is enriched in dgCoCat.

Let $\mathcal{A}, \mathcal{B}, \mathcal{C}$ be $A_{\infty}$-categories. There is a graded cocategory morphism of degree 0

$$
M: T s A_{\infty}(\mathcal{A}, \mathcal{B}) \otimes T s A_{\infty}(\mathcal{B}, \mathcal{C}) \rightarrow T s A_{\infty}(\mathcal{A}, \mathcal{C})
$$

defined in Section 4 via diagram (4.0.1). Since all cocategory morphisms $\alpha, \alpha \otimes 1$ in this diagram commute with the differential by Corollary 5.4, the cocategory morphism $M$ also commutes with the differential:

$$
(1 \otimes B+B \otimes 1) M=M B
$$

by Proposition 5.5. Therefore, $M$ is an $A_{\infty}$-functor. The unit $\eta_{\mathcal{A}}: T \mathbb{1} \rightarrow$ $T \operatorname{Coder}(s \mathcal{A}, s \mathcal{A}), 1 \mapsto \operatorname{id}_{\mathcal{A}}$ also is an $A_{\infty}$-functor for trivial reasons. The set

$$
\operatorname{dgCoCat}\left(T \mathbb{1}, T s A_{\infty}(\mathcal{A}, \mathcal{B})\right)=\operatorname{Maps}\left(\{*\}, \operatorname{Ob} A_{\infty}(\mathcal{A}, \mathcal{B})\right)=\operatorname{dgCoCat}(T s \mathcal{A}, T s \mathcal{B})
$$


is the set of $A_{\infty}$-functors $\mathcal{A} \rightarrow \mathcal{B}$. We summarize the above statements as follows: the category of $A_{\infty}$-categories is enriched in dgCoCat. Moreover, it is enriched in the monoidal subcategory of $\mathrm{dgCoCat}$ generated by $T s \mathcal{C}$, where $\mathcal{C}$ are $A_{\infty}$-categories.

Let us apply Proposition 5.5 to the $A_{\infty}$-functor $M$. From that result we deduce the existence of a unique $A_{\infty}$-functor

$$
A_{\infty}\left(\mathcal{A},{ }_{-}\right): A_{\infty}(\mathcal{B}, \mathcal{C}) \rightarrow A_{\infty}\left(A_{\infty}(\mathcal{A}, \mathcal{B}), A_{\infty}(\mathcal{A}, \mathcal{C})\right)
$$

such that

$$
\begin{aligned}
M= & {\left[T s A_{\infty}(\mathcal{A}, \mathcal{B}) \otimes T s A_{\infty}(\mathcal{B}, \mathcal{C}) \stackrel{1 \otimes A_{\infty}(\mathcal{A},-)}{\longrightarrow}\right.} \\
& \left.T s A_{\infty}(\mathcal{A}, \mathcal{B}) \otimes T s A_{\infty}\left(A_{\infty}(\mathcal{A}, \mathcal{B}), A_{\infty}(\mathcal{A}, \mathcal{C})\right) \stackrel{\alpha}{\longrightarrow} T s A_{\infty}(\mathcal{A}, \mathcal{C})\right] .
\end{aligned}
$$

Let us find the components of $A_{\infty}\left(\mathcal{A},{ }_{-}\right)$.

6.2 Proposition. The $A_{\infty}$-functor $A_{\infty}\left(\mathcal{A},,_{-}\right)$is strict. It maps an object of $A_{\infty}(\mathcal{B}, \mathcal{C})$, an $A_{\infty}$-functor $g: \mathcal{B} \rightarrow \mathcal{C}$, to the object of the target $A_{\infty}$-category $(1 \otimes g) M: A_{\infty}(\mathcal{A}, \mathcal{B}) \rightarrow A_{\infty}(\mathcal{A}, \mathcal{C})$ (which is also an $A_{\infty}$-functor). The first component $A_{\infty}\left(\mathcal{A},{ }_{-}\right)_{1}$ maps an element $t$ of $s A_{\infty}(\mathcal{B}, \mathcal{C})(g, h)$, a $(g, h)$-coderivation $t: T s \mathcal{B} \rightarrow T s \mathcal{C}$, to the $((1 \otimes g) M,(1 \otimes h) M)$-coderivation $(1 \otimes t) M: T s A_{\infty}(\mathcal{A}, \mathcal{B}) \rightarrow$ $T s A_{\infty}(\mathcal{A}, \mathcal{C})$, an element of $s A_{\infty}\left(A_{\infty}(\mathcal{A}, \mathcal{B}), A_{\infty}(\mathcal{A}, \mathcal{C})\right)((1 \otimes g) M,(1 \otimes h) M)$.

Proof. Clearly, $A_{\infty}\left(\mathcal{A},{ }_{-}\right)$gives the mapping of objects $g \mapsto(1 \otimes g) M$ as described. To prove that $A_{\infty}\left(\mathcal{A},{ }_{-}\right)_{1}: t \mapsto(1 \otimes t) M$ and $A_{\infty}\left(\mathcal{A},{ }_{-}\right)_{k}=0$ for $k>1$ it suffices to substitute a cocategory homomorphism with those components into (6.1.2) and to check this equation (see Proposition 3.4). Let

$$
\begin{aligned}
& p^{1} \otimes \cdots \otimes p^{n} \in s A_{\infty}(\mathcal{A}, \mathcal{B})\left(f^{0}, f^{1}\right) \otimes \cdots \otimes s A_{\infty}(\mathcal{A}, \mathcal{B})\left(f^{n-1}, f^{n}\right), \\
& t^{1} \otimes \cdots \otimes t^{m} \in s A_{\infty}(\mathcal{B}, \mathcal{C})\left(g^{0}, g^{1}\right) \otimes \cdots \otimes s A_{\infty}(\mathcal{B}, \mathcal{C})\left(g^{m-1}, g^{m}\right) .
\end{aligned}
$$

The equation to check is

$$
\left(p^{1} \otimes \cdots \otimes p^{n} \otimes t^{1} \otimes \cdots \otimes t^{m}\right) M=\left(p^{1} \otimes \cdots \otimes p^{n}\right) \cdot\left[\left(t^{1} \otimes \cdots \otimes t^{m}\right) A_{\infty}\left(\mathcal{A},{ }_{-}\right)\right] \theta,
$$

that is,

$$
\left(p^{1} \otimes \cdots \otimes p^{n} \otimes t^{1} \otimes \cdots \otimes t^{m}\right) M=\left(p^{1} \otimes \cdots \otimes p^{n}\right) .\left[\left(1 \otimes t^{1}\right) M \otimes \cdots \otimes\left(1 \otimes t^{m}\right) M\right] \theta .
$$

The left hand side is a cocategory homomorphism. Let us prove that the right hand side

$$
\left(p^{1} \otimes \cdots \otimes p^{n} \otimes t^{1} \otimes \cdots \otimes t^{m}\right) L \stackrel{\text { def }}{=}\left(p^{1} \otimes \cdots \otimes p^{n}\right) \cdot\left[\left(1 \otimes t^{1}\right) M \otimes \cdots \otimes\left(1 \otimes t^{m}\right) M\right] \theta
$$


is also a cocategory homomorphism. Indeed,

$$
\begin{aligned}
& \left(p^{1} \otimes \cdots \otimes p^{n} \otimes t^{1} \otimes \cdots \otimes t^{m}\right) L \Delta \\
& =\left(p^{1} \otimes \cdots \otimes p^{n}\right) \cdot\left[\left(1 \otimes t^{1}\right) M \otimes \cdots \otimes\left(1 \otimes t^{m}\right) M\right] \theta \Delta \\
& =\left(p^{1} \otimes \cdots \otimes p^{n}\right) \Delta \sum_{k=0}^{m}\left[\left(1 \otimes t^{1}\right) M \otimes \cdots \otimes\left(1 \otimes t^{k}\right) M\right] \theta \\
& \otimes\left[\left(1 \otimes t^{k+1}\right) M \otimes \cdots \otimes\left(1 \otimes t^{m}\right) M\right] \theta \\
& =\sum_{l=0}^{n} \sum_{k=0}^{m}(-)^{\left(p^{l+1}+\cdots+p^{n}\right)\left(t^{1}+\cdots+t^{k}\right)}\left(p^{1} \otimes \cdots \otimes p^{l}\right) \cdot\left[\left(1 \otimes t^{1}\right) M \otimes \cdots \otimes\left(1 \otimes t^{k}\right) M\right] \theta \\
& \otimes\left(p^{l+1} \otimes \cdots \otimes p^{n}\right) \cdot\left[\left(1 \otimes t^{k+1}\right) M\right. \\
& \left.\otimes \cdots \otimes\left(1 \otimes t^{m}\right) M\right] \theta \\
& =\left[\left(p^{1} \otimes \cdots \otimes p^{n}\right) \Delta \otimes\left(t^{1} \otimes \cdots \otimes t^{m}\right) \Delta\right](1 \otimes c \otimes 1)(L \otimes L)
\end{aligned}
$$

by Proposition 3.1 .

Let us prove that the components of $M$ and $L$ coincide. For $m=0$ and any $n>0$ we have

$$
\left(p^{1} \otimes \cdots \otimes p^{n} \mid g^{0}\right) L_{n 0}=\left(p^{1} \otimes \cdots \otimes p^{n}\right) .\left(1 \otimes g^{0}\right) M \mathrm{pr}_{1}=\left(p^{1} \otimes \cdots \otimes p^{n} \mid g^{0}\right) M_{n 0},
$$

hence, $L_{n 0}=M_{n 0}$. For $m=1$ and any $n \geqslant 0$ we have

$$
\left(p^{1} \otimes \cdots \otimes p^{n} \otimes t^{1}\right) L_{n 1}=\left(p^{1} \otimes \cdots \otimes p^{n}\right) \cdot\left(1 \otimes t^{1}\right) M \operatorname{pr}_{1}=\left(p^{1} \otimes \cdots \otimes p^{n} \otimes t^{1}\right) M_{n 1},
$$

hence, $L_{n 1}=M_{n 1}$. For $m>1$ and any $n \geqslant 0$ we have $L_{n m}=0$ and $M_{n m}=0$. Therefore, $L=M$ and the proposition is proved.

6.3 Corollary. For all $m>0$ and all $t^{1} \otimes \cdots \otimes t^{m}$ as in (6.2.1) we have

$$
\left[\left(1 \otimes t^{1}\right) M \otimes \cdots \otimes\left(1 \otimes t^{m}\right) M\right] \tilde{B}_{m}=\left[1 \otimes\left(t^{1} \otimes \cdots \otimes t^{m}\right) B_{m}\right] M,
$$

where $\tilde{B}$ denotes the differential in $T s A_{\infty}\left(A_{\infty}(\mathcal{A}, \mathcal{B}), A_{\infty}(\mathcal{A}, \mathcal{C})\right)$.

Indeed, the general property of an $A_{\infty}$-functor $A_{\infty}\left(\mathcal{A},{ }_{-}\right) \tilde{B}=B A_{\infty}\left(\mathcal{A},,_{-}\right)$reduces to the above formula, since $A_{\infty}\left(\mathcal{A},{ }_{-}\right)$is strict.

In the following definition we introduce $A_{\infty}$-analogs of natural transformations.

6.4 Definition ( $\omega$-globular set of $A_{\infty}$-categories). A natural $A_{\infty}$-transformation $r: f \rightarrow g: \mathcal{A} \rightarrow \mathcal{B}$ (natural transformation in terms of [Fuk]) is an $A_{\infty}$-transformation of degree -1 such that $r b+b r=0$ (that is, $(r) B_{1}=0$ ). The $\omega$-globular set [Bat98] $A_{\omega}$ of $A_{\infty}$-categories is defined as follows: objects (0-morphisms) are $A_{\infty}$-categories $\mathcal{A}$; 1-morphisms are $A_{\infty}$-functors $f: \mathcal{A} \rightarrow \mathcal{B} ; 2$-morphisms are natural $A_{\infty}$-transformations $r: f \rightarrow g: \mathcal{A} \rightarrow \mathcal{B} ; 3$-morphisms $\lambda: r \rightarrow s: f \rightarrow g: \mathcal{A} \rightarrow \mathcal{B}$ are $(f, g)$-coderivations of degree -2 , such that $r-s=[\lambda, b]$; for $n \geqslant 3 n$-morphisms $\lambda_{n}: \lambda_{n-1} \rightarrow \mu_{n-1}: \cdots: r \rightarrow s: f \rightarrow g: \mathcal{A} \rightarrow \mathcal{B}$ are $(f, g)$-coderivations of degree $1-n$, such that $\lambda_{n-1}-\mu_{n-1}=\left[\lambda_{n}, b\right]$ (notice that the both sides are $(f, g)$-coderivations of degree $2-n)$. 
6.5 Remark. Let us notice that the $A_{\infty}$-functor $A_{\infty}\left(\mathcal{A},,_{-}\right)$from Proposition 6.2 defines a map of the $\omega$-globular set $A_{\omega}$ into itself. Indeed, objects $\mathcal{B}$ of $A_{\omega}$ are mapped into objects $A_{\infty}(\mathcal{A}, \mathcal{B}), 1$-morphisms $g: \mathcal{B} \rightarrow \mathcal{C}$ are mapped into 1-morphisms $(1 \otimes g) M: A_{\infty}(\mathcal{A}, \mathcal{B}) \rightarrow A_{\infty}(\mathcal{A}, \mathcal{C})$ and the first component $A_{\infty}\left(\mathcal{A},{ }_{-}\right)_{1}$ maps $(g, h)$-coderivations into $((1 \otimes g) M,(1 \otimes h) M)$-coderivations. Moreover, if the equation $\lambda_{n-1}-\mu_{n-1}=\lambda_{n} B_{1}$ holds for $(g, h)$-coderivations, then $\left(1 \otimes \lambda_{n-1}\right) M-(1 \otimes$ $\left.\mu_{n-1}\right) M=\left(1 \otimes \lambda_{n}\right) M \tilde{B}_{1}$ by $(6.3 .1)$, so the sources and the targets are preserved.

It might be useful to turn the $\omega$-globular set $A_{\omega}$ into a weak non-unital $\omega$-category in the sense of some of the existing definitions of the latter. Plenty of such definitions including [Bat98] are listed in Leinster's survey [Lei02]. We do not try to proceed in this direction. Instead we truncate the $\omega$-globular set to a 2 -globular set (that is, we deal with 0-, 1- and 2-morphisms) and we make a 2-category out of it.

\section{2-categories of $A_{\infty}$-categories}

Let $\mathcal{K}$ denote the category $\mathrm{K}(\mathbb{k}$-mod $)=H^{0}(\mathrm{C}(\mathbb{k}-\bmod ))$ of differential graded complexes of $\mathbb{k}$-modules, whose morphisms are chain maps modulo homotopy. Equipped with the usual tensor product, the unit object $\mathbb{k}$ and the standard symmetry, $\mathcal{K}$ becomes a $\mathbb{k}$-linear closed monoidal symmetric category. The inner hom-object is the usual $\operatorname{Hom}_{\mathfrak{k}}^{\cdot}(-,-)$. There is a notion of a category $\mathcal{C}$ enriched in $\mathcal{K}(\mathcal{K}$-categories, $\mathcal{K}$-functors, $\mathcal{K}$-natural transformations), see Kelly [Kel82]: for all objects $X, Y$ of $\mathcal{C}$ $\mathcal{C}(X, Y)$ is an object of $\mathcal{K}$. There is a similar notion of a 2-category enriched in $\mathcal{K}$, or a $\mathcal{K}$-2-category: it consist of a class of objects $\mathrm{Ob} \mathcal{C}$, a class of 1-morphisms $\mathrm{C}(X, Y)$ for each pair of objects $X, Y$ of $\mathcal{A}$, an object of 2-morphisms $\mathcal{C}(X, Y)(f, g) \in \mathrm{Ob} \mathcal{K}$ for each pair of 1-morphisms $f, g \in \mathfrak{C}(X, Y)$ and other data. We shall consider 1-unital, non-2-unital $\mathcal{K}$-2-categories. They are equipped with the following operations: associative composition of 1-morphisms, commuting left and right associative actions of 1-morphisms on 2-morphisms (these actions are morphisms in $\mathcal{K}$ ), 1-units, associative vertical composition of 2 -morphisms (a morphism in $\mathcal{K}$ ) compatible with the left and right actions of 1-morphisms on 2-morphisms and such that the both ways to obtain the horizontal composition coincide. Precise definitions are given in Appendix A.

7.1 Proposition. The following data define a 1-unital, non-2-unital $\mathcal{K}$-2-category $\mathcal{K} A_{\infty}$ :

- objects are $A_{\infty}$-categories;

- 1-morphisms are $A_{\infty}$-functors;

- an object of 2-morphisms between $f, g: \mathcal{A} \rightarrow \mathcal{B}$ is $\left(A_{\infty}(\mathcal{A}, \mathcal{B})(f, g), m_{1}\right) \in$ Ob $\mathcal{K}, m_{1}=s B_{1} s^{-1}$;

- the composition of 1-morphisms is the composition of $A_{\infty}$-functors;

- unit 1-morphisms are identity $A_{\infty}$-functors;

- the right action of a 1-morphism $k: \mathcal{B} \rightarrow \mathcal{C}$ on 2-morphisms is the chain map $\left(A_{\infty}(\mathcal{A}, \mathcal{B})(f, g), m_{1}\right) \rightarrow\left(A_{\infty}(\mathcal{A}, \mathcal{C})(f k, g k), m_{1}\right), r s^{-1} \mapsto\left(r s^{-1}\right) \cdot k=(r k) s^{-1}$, where $r$ is an $(f, g)$-coderivation; 
- the left action of a 1-morphism $e: \mathcal{D} \rightarrow \mathcal{A}$ on 2-morphisms is the chain map $\left(A_{\infty}(\mathcal{A}, \mathcal{B})(f, g), m_{1}\right) \rightarrow\left(A_{\infty}(\mathcal{D}, \mathcal{B})(e f, e g), m_{1}\right), r s^{-1} \mapsto e \cdot\left(r s^{-1}\right)=(e r) s^{-1}$, where $r$ is an $(f, g)$-coderivation;

- the vertical composition is the chain map $m_{2}=(s \otimes s) B_{2} s^{-1}: A_{\infty}(\mathcal{A}, \mathcal{B})(f, g) \otimes$ $A_{\infty}(\mathcal{A}, \mathcal{B})(g, h) \rightarrow A_{\infty}(\mathcal{A}, \mathcal{B})(f, h)$.

Proof. Clearly, the composition of 1-morphisms and the actions of 1-morphisms on 2 -morphisms are associative. The right and the left actions are unital, and commute with each other. The equation $-\left(1 \otimes m_{1}+m_{1} \otimes 1\right) m_{2}+m_{2} m_{1}=0$ (see (2.3.2) for $k=2)$ shows that $m_{2}$ is a chain map. The equation

$$
m_{3} m_{1}+\left(1 \otimes 1 \otimes m_{1}+1 \otimes m_{1} \otimes 1+m_{1} \otimes 1 \otimes 1\right) m_{3}-\left(m_{2} \otimes 1\right) m_{2}+\left(1 \otimes m_{2}\right) m_{2}=0
$$

(see (2.3.2) for $k=3$ ) shows that $m_{2}$ is associative in $\mathcal{K}$.

Let us check that the vertical composition is compatible with the actions of 1-morphisms on 2-morphisms. Applying equation (6.1.1) to $r \otimes p \otimes 1 \in s A_{\infty}(\mathcal{A}, \mathcal{B})(f, g) \otimes$ $s A_{\infty}(\mathcal{A}, \mathcal{B})(g, h) \otimes \mathbb{k} \subset T^{2} s A_{\infty}(\mathcal{A}, \mathcal{B})(f, h) \otimes T^{0} s A_{\infty}(\mathcal{B}, \mathcal{C})(k, k)$ we find that

$$
(r \otimes p \mid k) M_{20} B_{1}+(r k \otimes p k) B_{2}=\left[(r \otimes p)\left(1 \otimes B_{1}+B_{1} \otimes 1\right) \mid k\right] M_{20}+(r \otimes p) B_{2} k .
$$

One deduces that $\left(r s^{-1} \cdot k \otimes p s^{-1} \cdot k\right) m_{2}=\left(r s^{-1} \otimes p s^{-1}\right) m_{2} \cdot k$ in $\mathcal{K}$. Applying equation (6.1.1) to $1 \otimes r \otimes p \in \mathbb{k} \otimes s A_{\infty}(\mathcal{A}, \mathcal{B})(f, g) \otimes s A_{\infty}(\mathcal{A}, \mathcal{B})(g, h) \subset$ $T^{0} s A_{\infty}(\mathcal{D}, \mathcal{A})(e, e) \otimes T^{2} s A_{\infty}(\mathcal{A}, \mathcal{B})(f, h)$ we find that

$$
\begin{aligned}
& (e r \otimes e p) B_{2}=(e \mid r \otimes p) M_{02} B_{1}+(e r \otimes e p) B_{2} \\
& \quad=\left[e \mid(r \otimes p)\left(1 \otimes B_{1}+B_{1} \otimes 1\right)\right] M_{02}+e(r \otimes p) B_{2}=e(r \otimes p) B_{2}
\end{aligned}
$$

(notice that $\left.M_{02}=0\right)$. Therefore, $\left(e \cdot r s^{-1} \otimes e \cdot p s^{-1}\right) m_{2}=e \cdot\left(r s^{-1} \otimes p s^{-1}\right) m_{2}$.

Now let us prove distributivity. Applying equation (6.1.1) to $r \otimes p \in$ $s A_{\infty}(\mathcal{A}, \mathcal{B})(f, g) \otimes s A_{\infty}(\mathcal{B}, \mathcal{C})(h, k)$ we find that

$(r h \otimes g p) B_{2}+(-)^{r p}(f p \otimes r k) B_{2}+(r \otimes p) M_{11} B_{1}=(r \otimes p)\left(1 \otimes B_{1}+B_{1} \otimes 1\right) M_{11}$.

Thus, $(r h \otimes g p) B_{2}+(-)^{r p}(f p \otimes r k) B_{2}=0$ in $\mathcal{K}$. We deduce that modulo homotopy

$$
\begin{gathered}
\left(r s^{-1} \cdot h \otimes g \cdot p s^{-1}\right) m_{2} s=\left(r h s^{-1} \otimes g p s^{-1}\right)(s \otimes s) B_{2}=(-)^{p+1}(r h \otimes g p) B_{2} \\
=(-)^{r p+p}(f p \otimes r k) B_{2}=(-)^{r p+p}\left(f p s^{-1} s \otimes r k s^{-1} s\right) B_{2} \\
=(-)^{r p+p+r+1}\left(f \cdot p s^{-1} \otimes r s^{-1} \cdot k\right) m_{2} s .
\end{gathered}
$$

Therefore, $\left(r s^{-1} \cdot h \otimes g \cdot p s^{-1}\right) m_{2}=(-)^{(r+1)(p+1)}\left(f \cdot p s^{-1} \otimes r s^{-1} \cdot k\right) m_{2}$ in $\mathcal{K}$, as stated.

The 0-th cohomology functor $H^{0}=\mathcal{K}\left(\mathbb{k},{ }_{-}\right): \mathcal{K} \rightarrow \mathbb{k}$-mod, $X \mapsto H^{0}(X)=$ $\mathcal{K}(\mathbb{k}, X)$ is lax monoidal symmetric, since the complex $\mathbb{k}$ concentrated in degree 0 is the unit object of $\mathcal{K}$. It determines a functor $H^{0}: \mathcal{K}$-Cat $\rightarrow \mathbb{k}$-Cat. To a $\mathcal{K}$-category $\mathcal{C}$ it assigns a $\mathbb{k}$-linear category $H^{0}(\mathcal{C})$ with the same class of objects, and for each pair $X, Y$ of objects of $\mathcal{C}$ the $\mathbb{k}$-module $H^{0}(\mathcal{C})(X, Y)=H^{0}(\mathcal{C}(X, Y))$. The functor $H^{0}: \mathcal{K}-\mathrm{C} a t \rightarrow \mathbb{k}-\mathrm{C} a t$ is also lax monoidal symmetric. Therefore, there is a functor $\mathcal{K}$-Cat-Cat $\rightarrow \mathrm{C} a t$-Cat, again denoted $H^{0}$ by abuse of notation, and the corresponding functor $\mathcal{K}-2-\mathrm{C} a t^{n u} \rightarrow 2-\mathrm{C} a t^{n u}$. See Appendix A for the definition of 
1-unital, non-2-unital $\mathcal{K}$ - or $\mathbb{k}$ - 2-categories. To $\mathcal{K} A_{\infty}$ the functor assigns a 1 -unital, non-2-unital $\mathbb{k}$-linear 2-category $A_{\infty}$. Let us describe it in detail. Objects of $A_{\infty}$ are $A_{\infty}$-categories, 1-morphisms are $A_{\infty}$-functors, and 2-morphisms are elements of

$$
H^{0}\left(A_{\infty}(\mathcal{A}, \mathcal{B})(f, g), m_{1}\right) \stackrel{s}{\sim} H^{-1}\left(s A_{\infty}(\mathcal{A}, \mathcal{B})(f, g), B_{1}\right)
$$

that is, equivalence classes of natural $A_{\infty}$-transformations $r: f \rightarrow g: \mathcal{A} \rightarrow \mathcal{B}$. Natural $A_{\infty}$-transformations $r, t: f \rightarrow g: \mathcal{A} \rightarrow \mathcal{B}$ are equivalent, if they are connected by a 3-morphism $\lambda: r \rightarrow t$, that is, $r-t=\lambda B_{1}$. Both compositions of 1-morphisms with 2-morphisms $\operatorname{Mor}_{2}(\mathcal{A}, \mathcal{B}) \times \operatorname{Mor}_{1}(\mathcal{B}, \mathcal{C}) \rightarrow \operatorname{Mor}_{2}(\mathcal{A}, \mathcal{C}),(r, h) \mapsto$ $r h$ and $\operatorname{Mor}_{1}(\mathcal{A}, \mathcal{B}) \times \operatorname{Mor}_{2}(\mathcal{B}, \mathcal{C}) \rightarrow \operatorname{Mor}_{2}(\mathcal{A}, \mathcal{C}),(f, p) \mapsto f p$ are compositions of $\mathbb{k}$-linear maps $T s \mathcal{A} \rightarrow T s \mathcal{B} \rightarrow T s \mathcal{C}$. The vertical composition $m_{2}$ of 2-morphisms, translated to $H^{-1}\left(s A_{\infty}\right)$ assigns the natural $A_{\infty}$-transformation $r \cdot p=(r \otimes p) B_{2}$ to natural $A_{\infty}$-transformations $r: f \rightarrow g$ and $p: g \rightarrow h$. Indeed, $\left(r s^{-1} \otimes p s^{-1}\right) m_{2} s=$ $\left(r s^{-1} \otimes p s^{-1}\right)(s \otimes s) B_{2}=(r \otimes p) B_{2}$. Compatibility of these constructions with the equivalence relation is obvious from the construction, and can be verified directly.

7.2 Remark. Let $\mathcal{A}$ be an $A_{\infty}$-category. It determines a map $A_{\omega} \rightarrow A_{\omega}$, described in Remark 6.5. This map restricts to a map $A_{\infty}\left(\mathcal{A},_{-}\right): A_{\infty} \rightarrow A_{\infty}$. It takes an $A_{\infty}$-category $\mathcal{B}$ to the $A_{\infty}$-category $A_{\infty}(\mathcal{A}, \mathcal{B})$, an $A_{\infty}$-functor $g: \mathcal{B} \rightarrow \mathcal{C}$ to the $A_{\infty}$-functor $(1 \otimes g) M: A_{\infty}(\mathcal{A}, \mathcal{B}) \rightarrow A_{\infty}(\mathcal{A}, \mathcal{C})$, and an equivalence class of a natural $A_{\infty}$-transformation $t: g \rightarrow h: \mathcal{A} \rightarrow \mathcal{B}$ to the equivalence class of the natural $A_{\infty}$-transformation $(1 \otimes t) M:(1 \otimes g) M \rightarrow(1 \otimes h) M: A_{\infty}(\mathcal{A}, \mathcal{B}) \rightarrow A_{\infty}(\mathcal{A}, \mathrm{C})$, see Remark 6.5. Let us prove that $A_{\infty}\left(\mathcal{A},_{-}\right): A_{\infty} \rightarrow A_{\infty}$ is a strict 2 -functor. Indeed, it preserves the composition of 1-morphisms, $(1 \otimes f) M(1 \otimes g) M=(1 \otimes f g) M$, and the both compositions of 1-morphisms and 2-morphisms, $(1 \otimes f) M(1 \otimes t) M=(1 \otimes f t) M$, $(1 \otimes t) M(1 \otimes f) M=(1 \otimes t f) M$, due to associativity of $M$. The vertical composition of 2-morphisms is preserved due to (6.3.1) for $m=2$ :

$$
\left[\left(1 \otimes t^{1}\right) M \otimes\left(1 \otimes t^{2}\right) M\right] \tilde{B}_{2}=\left[1 \otimes\left(t^{1} \otimes t^{2}\right) B_{2}\right] M
$$

7.3 Definition (Unital $A_{\infty}$-categories). Let $\mathcal{C}$ be an $A_{\infty}$-category. It is called unital if for each object $X$ of $\mathcal{C}$ there is a unit element - a $\mathbb{k}$-linear map ${ }_{X} \mathbf{i}_{0}^{\mathcal{C}}: \mathbb{k} \rightarrow$ $(s \mathcal{C})^{-1}(X, X)$ such that ${ }_{X} \mathbf{i}_{0}^{\mathrm{C}} b_{1}=0,\left({ }_{X} \mathbf{i}_{0}^{\mathrm{C}} \otimes{ }_{X} \mathbf{i}_{0}^{\mathrm{C}}\right) b_{2}-{ }_{X} \mathbf{i}_{0}^{\mathrm{C}} \in \operatorname{Im} b_{1}$, and for all pairs $X, Y$ of objects of $\mathcal{C}$ the chain maps $\left(1 \otimes_{Y} \mathbf{i}_{0}^{\mathcal{C}}\right) b_{2},\left({ }_{X} \mathbf{i}_{0}^{\mathrm{e}} \otimes 1\right) b_{2}: s \mathcal{C}(X, Y) \rightarrow s \mathcal{C}(X, Y)$ are homotopy invertible.

In particular, an $A_{\infty}$-algebra $\mathcal{C}$ is unital if it has an element $\mathbf{i}_{0}^{\mathrm{C}} \in(s \mathcal{C})^{-1}$ such that $\mathbf{i}_{0}^{\mathrm{e}} b_{1}=0,\left(\mathbf{i}_{0}^{\mathrm{e}} \otimes \mathbf{i}_{0}^{\mathrm{e}}\right) b_{2}-\mathbf{i}_{0}^{\mathrm{e}} \in \operatorname{Im} b_{1}$, and the chain maps $\left(1 \otimes \mathbf{i}_{0}^{\mathrm{e}}\right) b_{2},\left(\mathbf{i}_{0}^{\mathrm{C}} \otimes 1\right) b_{2}: s \mathrm{C} \rightarrow s \mathcal{C}$ are homotopy invertible. Our definition differs from a that of a homological unit (e.g. [LH02]) by the last invertibility condition. It produces rather a homotopical unit:

7.4 Lemma. Let ${ }_{X} \mathbf{i}_{0}^{\mathrm{e}}$ be as in Definition 7.3 of a unital $A_{\infty}$-category $\mathcal{C}$, then for each pair $X, Y$ of objects of $\mathcal{C}$ we have

$$
\begin{gathered}
\left(1 \otimes{ }_{Y} \mathbf{i}_{0}^{\mathrm{e}}\right) b_{2} \sim 1: s \mathrm{C}(X, Y) \rightarrow s \mathrm{e}(X, Y), \\
\left({ }_{X} \mathbf{i}_{0}^{\mathrm{C}} \otimes 1\right) b_{2} \sim-1: s \mathrm{C}(X, Y) \rightarrow s \mathrm{C}(X, Y) .
\end{gathered}
$$


Proof. For each object $X$ of $\mathcal{C}$ there is a $\mathbb{k}$-linear map ${ }_{X} v_{0}: \mathbb{k} \rightarrow(s \mathcal{C})^{-2}(X, X)$ such that $\left({ }_{X} \mathbf{i}_{0}^{\mathrm{e}} \otimes{ }_{X} \mathbf{i}_{0}^{\mathrm{e}}\right) b_{2}-{ }_{X} \mathbf{i}_{0}^{\mathrm{e}}={ }_{X} v_{0} b_{1}$. Hence,

$$
\begin{aligned}
& \left(X_{X} \mathbf{i}_{0}^{\mathrm{e}} \otimes 1\right) b_{2}\left({ }_{X} \mathbf{i}_{0}^{\mathrm{e}} \otimes 1\right) b_{2}=\left({ }_{X} \mathbf{i}_{0}^{\mathrm{e}} \otimes X_{X} \mathbf{i}_{0}^{\mathrm{e}} \otimes 1\right)\left(1 \otimes b_{2}\right) b_{2} \underset{k=3}{\stackrel{(2.3 .1)}{\sim}}-\left(X_{X} \mathbf{i}_{0}^{\mathrm{e}} \otimes X_{X} \mathbf{i}_{0}^{\mathrm{e}} \otimes 1\right)\left(b_{2} \otimes 1\right) b_{2} \\
& =-\left[\left({ }_{X} \mathbf{i}_{0}^{\mathrm{e}} \otimes{ }_{X} \mathbf{i}_{0}^{\mathrm{e}}\right) b_{2} \otimes 1\right] b_{2}=-\left({ }_{X} \mathbf{i}_{0}^{\mathrm{e}} \otimes 1\right) b_{2}-\left({ }_{X} v_{0} b_{1} \otimes 1\right) b_{2} \\
& =-\left(x \mathbf{i}_{0}^{\mathrm{e}} \otimes 1\right) b_{2}+b_{1}\left(x v_{0} \otimes 1\right) b_{2}+\left(x v_{0} \otimes 1\right) b_{2} b_{1} \sim-\left({ }_{x} \mathbf{i}_{0}^{\mathrm{e}} \otimes 1\right) b_{2}, \\
& \left(1 \otimes_{Y} \mathbf{i}_{0}^{\mathfrak{e}}\right) b_{2}\left(1 \otimes_{Y} \mathbf{i}_{0}^{\mathbf{e}}\right) b_{2}=-\left(1 \otimes_{Y} \mathbf{i}_{0}^{\mathbf{e}} \otimes_{Y} \mathbf{i}_{0}^{\mathfrak{e}}\right)\left(b_{2} \otimes 1\right) b_{2} \underset{k=3}{\stackrel{(2.3 .1)}{r}}\left(1 \otimes_{Y} \mathbf{i}_{0}^{\mathfrak{e}} \otimes_{Y} \mathbf{i}_{0}^{\mathfrak{e}}\right)\left(1 \otimes b_{2}\right) b_{2} \\
& =\left[1 \otimes\left({ }_{Y} \mathbf{i}_{0}^{\mathfrak{e}} \otimes_{Y} \mathbf{i}_{0}^{\mathfrak{e}}\right) b_{2}\right] b_{2}=\left(1 \otimes_{Y} \mathbf{i}_{0}^{\mathfrak{e}}\right) b_{2}+\left(1 \otimes_{Y} v_{0} b_{1}\right) b_{2} \\
& =\left(1 \otimes_{Y} \mathbf{i}_{0}^{\mathfrak{e}}\right) b_{2}-b_{1}\left(1 \otimes_{Y} v_{0}\right) b_{2}-\left(1 \otimes_{Y} v_{0}\right) b_{2} b_{1} \sim\left(1 \otimes_{Y} \mathbf{i}_{0}^{\mathfrak{e}}\right) b_{2} .
\end{aligned}
$$

We see that $-\left({ }_{X} \mathbf{i}_{0}^{\mathrm{e}} \otimes 1\right) b_{2}$ and $\left(1 \otimes_{Y} \mathbf{i}_{0}^{\mathrm{e}}\right) b_{2}$ are invertible idempotents in $\mathcal{K}$. Therefore, these maps are both homotopic to the identity map.

This lemma shows that a unital $A_{\infty}$-algebra may be defined as an $A_{\infty}$-algebra $\mathcal{C}$, such that the graded associative $\mathbb{k}$-algebra $H^{\bullet}\left(\mathcal{C}, m_{1}\right)$ has a unit $1 \in H^{0}\left(\mathcal{C}, m_{1}\right)$ and for some/any representative $1^{\mathcal{C}} \in \mathcal{C}^{0}$ of the class $1 \in H^{0}\left(\mathcal{C}, m_{1}\right)$ the chain maps $\left(\mathrm{id} \otimes 1^{\mathcal{C}}\right) m_{2},\left(1^{\mathcal{C}} \otimes \mathrm{id}\right) m_{2}: \mathcal{C} \rightarrow \mathcal{C}$ are homotopic to ide. A unit element $\mathbf{i}_{0}^{\mathfrak{C}} \in(s \mathcal{C})^{-1}$ corresponds to a unit $1^{\mathrm{e}} \in \mathrm{C}^{0}$ via $1^{\mathrm{e}} s=\mathbf{i}_{0}^{\mathrm{e}}$.

7.5 Proposition. Let $\mathcal{C}$ be a unital $A_{\infty}$-category. Then the collection ${ }_{X} \mathbf{i}_{0}^{\mathrm{e}}$ extends to a natural $A_{\infty}$-transformation $\mathbf{i}^{\mathrm{e}}: \operatorname{id}_{\mathcal{e}} \rightarrow \operatorname{id} \mathcal{C}: \mathcal{C} \rightarrow \mathcal{C}$ such that $\left(\mathbf{i}^{\mathrm{e}} \otimes \mathbf{i}^{\mathrm{e}}\right) B_{2} \equiv \mathbf{i}^{\mathrm{e}}$.

Proof. Let $\mathbb{k}$-linear maps $x v_{0}: \mathbb{k} \rightarrow(s \mathcal{C})^{-2}(X, X)$ satisfy the equations $\left(X \mathbf{i}_{0}^{\mathcal{e}} \otimes\right.$ $\left.{ }_{X} \mathbf{i}_{0}^{\mathrm{e}}\right) b_{2}-{ }_{X} \mathbf{i}_{0}^{\mathrm{e}}={ }_{X} v_{0} b_{1}$. We will prove that given $X_{X} \mathbf{i}_{0}^{\mathrm{e}},{ }_{X} v_{0}\left(\right.$ with $\left.{ }_{X} \mathbf{i}_{0}^{\mathrm{e}} b_{1}=0\right)$ are 0 -th components of a natural $A_{\infty}$-transformation $\mathbf{i}^{\mathrm{e}}$ and a 3 -morphism $v$ as follows:

$$
\begin{gathered}
\mathbf{i}^{\mathcal{E}}: \operatorname{id}_{\mathcal{C}} \rightarrow \operatorname{id}_{\mathcal{C}}: \mathcal{C} \rightarrow \mathcal{C}, \\
v:\left(\mathbf{i}^{\mathfrak{e}} \otimes \mathbf{i}^{\mathfrak{C}}\right) B_{2} \rightarrow \mathbf{i}^{\mathfrak{e}}: \text { ide }_{\mathcal{C}} \rightarrow \text { ide }_{\mathcal{C}}: \mathcal{C} \rightarrow \mathcal{C} .
\end{gathered}
$$

That is, we will prove the existence of $(1,1)$-coderivations $\mathbf{i}^{\mathrm{C}}, v: T s \mathcal{C} \rightarrow T s \mathcal{C}$ of degree -1 (resp. -2 ) such that

$$
\begin{aligned}
\mathbf{i}^{\mathfrak{e}} b+b \mathbf{i}^{\mathrm{e}} & =0, \\
\left(\mathbf{i}^{\mathrm{C}} \otimes \mathbf{i}^{\mathrm{e}}\right) B_{2}-\mathbf{i}^{\mathrm{e}} & =v b-b v .
\end{aligned}
$$

We already have the 0 -th components $\mathbf{i}_{0}^{\mathrm{e}}, v_{0}$. Let us construct the other components of $\mathbf{i}^{\mathrm{e}}$ and $v$ by induction. Given a positive $n$, assume that we have already found components $\mathbf{i}_{m}^{\mathrm{e}}, v_{m}$ of the sought $\mathbf{i}^{\mathrm{e}}, v$ for $m<n$, such that the equations

$$
\begin{aligned}
\left(\mathbf{i}^{\mathrm{e}} b\right)_{m}+\left(b \mathbf{i}^{\mathrm{e}}\right)_{m}=0: & s \mathcal{C}\left(X_{0}, X_{1}\right) \otimes \cdots \otimes s \mathfrak{C}\left(X_{m-1}, X_{m}\right) \rightarrow s \mathcal{C}\left(X_{0}, X_{m}\right), \\
{\left[\left(\mathbf{i}^{\mathrm{e}} \otimes \mathbf{i}^{\mathrm{e}}\right) B_{2}\right]_{m}-\mathbf{i}_{m}^{\mathrm{e}}=} & (v b)_{m}-(b v)_{m}: \\
& s \mathcal{C}\left(X_{0}, X_{1}\right) \otimes \cdots \otimes s \mathcal{C}\left(X_{m-1}, X_{m}\right) \rightarrow s \mathfrak{C}\left(X_{0}, X_{m}\right)
\end{aligned}
$$

are satisfied for all $m<n$. Here $(f)_{m}=\left(T^{m} s \mathcal{C} \hookrightarrow T s \mathcal{P} \stackrel{f}{\longrightarrow} T s \stackrel{\mathrm{pr}_{1}}{\longrightarrow} s \mathcal{C}\right)$ for an arbitrary morphism of quivers $f: T s \mathrm{C} \rightarrow T s \mathcal{C}$. 
Introduce (1,1)-coderivations $\tilde{\mathbf{i}}, v: T s \mathcal{C} \rightarrow T s \mathcal{C}$ of degree -1 and -2 by their components $\left(\mathbf{i}_{0}^{\mathrm{e}}, \mathbf{i}_{1}^{\mathrm{e}}, \ldots, \mathbf{i}_{n-1}^{\mathrm{e}}, 0,0, \ldots\right)\left(\operatorname{resp} .\left(v_{0}, v_{1}, \ldots, v_{n-1}, 0,0, \ldots\right)\right)$. Define (1,1)-coderivations $\lambda=\tilde{\mathbf{i}} b+b \tilde{\mathbf{i}}$ of degree 0 and $\nu=(\tilde{\mathbf{i}} \otimes \tilde{\mathbf{i}}) B_{2}-\tilde{\mathbf{i}}-\tilde{v} B_{1}$ of degree -1 . Then equations (7.5.1), (7.5.2) imply that $\lambda_{m}=0, \nu_{m}=0$ for $m<n$. The identity $\lambda b-b \lambda=0$ implies that

$$
\lambda_{n} d=\lambda_{n} b_{1}-\sum_{q+1+t=n}\left(1^{\otimes q} \otimes b_{1} \otimes 1^{\otimes t}\right) \lambda_{n}=0 .
$$

The $n$-th component of the identity

$\nu B_{1}=(\tilde{\mathbf{i}} \otimes \tilde{\mathbf{i}}) B_{2} B_{1}-\tilde{\mathbf{i}} B_{1}=-(\tilde{\mathbf{i}} \otimes \tilde{\mathbf{i}})\left(1 \otimes B_{1}+B_{1} \otimes 1\right) B_{2}-\lambda=-(\tilde{\mathbf{i}} \otimes \lambda) B_{2}+(\lambda \otimes \tilde{\mathbf{i}}) B_{2}-\lambda$ gives

$$
\begin{aligned}
\nu_{n} d & =\nu_{n} b_{1}+\sum_{q+1+t=n}\left(1^{\otimes q} \otimes b_{1} \otimes 1^{\otimes t}\right) \nu_{n}=-\left(\mathbf{i}_{0}^{\mathfrak{e}} \otimes \lambda_{n}\right) b_{2}+\left(\lambda_{n} \otimes \mathbf{i}_{0}^{\mathrm{e}}\right) b_{2}-\lambda_{n} \\
& =-\lambda_{n}\left(\mathbf{i}_{0}^{\mathfrak{e}} \otimes 1\right) b_{2}+\lambda_{n}\left(1 \otimes \mathbf{i}_{0}^{\mathfrak{e}}\right) b_{2}-\lambda_{n}=-\lambda_{n} u^{\prime} .
\end{aligned}
$$

Here the chain map

$$
u^{\prime}=\left(x_{0} \mathbf{i}_{0}^{\mathrm{e}} \otimes 1\right) b_{2}-\left(1 \otimes X_{n} \mathbf{i}_{0}^{\mathrm{e}}\right) b_{2}+1: s \mathcal{C}\left(X_{0}, X_{n}\right) \rightarrow s \mathcal{C}\left(X_{0}, X_{n}\right)
$$

is homotopic to -1 by Lemma 7.4. Hence, the map

$$
u=\operatorname{Hom}\left(N, u^{\prime}\right): \operatorname{Hom}^{\bullet}\left(N, s \mathcal{C}\left(X_{0}, X_{n}\right)\right) \rightarrow \operatorname{Hom}^{\bullet}\left(N, s \mathcal{C}\left(X_{0}, X_{n}\right)\right), \quad \lambda_{n} \mapsto \lambda_{n} u^{\prime}
$$

is also homotopic to -1 for each complex of $\mathbb{k}$-modules $N$, in particular, for $N=$ $s \mathfrak{C}\left(X_{0}, X_{1}\right) \otimes_{\mathbb{k}} \cdots \otimes_{\mathbb{k}} s \mathfrak{C}\left(X_{n-1}, X_{n}\right)$. Therefore, the complex Cone $(u)$ is contractible by Lemma Appendix B.1. Since $-\lambda_{n} d=0$ and $\nu_{n} d+\lambda_{n} u=0$, the element

$$
\left(\nu_{n}, \lambda_{n}\right) \in \operatorname{Hom}_{\mathbb{k}}^{-1}\left(N, s \mathcal{C}\left(X_{0}, X_{n}\right)\right) \oplus \operatorname{Hom}_{\mathbb{k}}^{0}\left(N, s \mathcal{C}\left(X_{0}, X_{n}\right)\right)=\operatorname{Cone}^{-1}(u)
$$

is a cycle. Due to acyclicity of Cone $(u)$ this element is a boundary of some element

$$
\left(v_{n}, \mathbf{i}_{n}^{\mathfrak{e}}\right) \in \operatorname{Hom}_{\mathbb{k}}^{-2}\left(N, s \mathcal{C}\left(X_{0}, X_{n}\right)\right) \oplus \operatorname{Hom}_{\mathbb{k}}^{-1}\left(N, s \mathcal{C}\left(X_{0}, X_{n}\right)\right)=\operatorname{Cone}^{-2}(u),
$$

that is, $v_{n} d+\mathbf{i}_{n}^{\mathrm{e}} u=\nu_{n}$ and $-\mathbf{i}_{n}^{\mathrm{e}} d=\lambda_{n}$. These equations can be rewritten as follows:

$$
\begin{aligned}
&-\mathbf{i}_{n}^{\mathfrak{e}} b_{1}-\sum_{q+1+t=n}\left(1^{\otimes q} \otimes b_{1} \otimes 1^{\otimes t}\right) \mathbf{i}_{n}^{\mathcal{e}}=(\tilde{\mathbf{i}} b)_{n}+(b \tilde{\mathbf{i}})_{n}, \\
& v_{n} b_{1}-\sum_{q+1+t=n}\left(1^{\otimes q} \otimes b_{1} \otimes 1^{\otimes t}\right) v_{n}-\left(\mathbf{i}_{0}^{\mathfrak{e}} \otimes \mathbf{i}_{n}^{\mathfrak{e}}\right) b_{2}-\left(\mathbf{i}_{n}^{\mathcal{C}} \otimes \mathbf{i}_{0}^{\mathfrak{e}}\right) b_{2}+\mathbf{i}_{n}^{\mathcal{e}} \\
&=\left[(\tilde{\mathbf{i}} \otimes \tilde{\mathbf{i}}) B_{2}\right]_{n}-(\tilde{v} b)_{n}+(b \tilde{v})_{n} .
\end{aligned}
$$

In other words, $(1,1)$-coderivations with components $\left(\mathbf{i}_{0}^{\mathrm{e}}, \ldots, \mathbf{i}_{n-1}^{\mathrm{e}}, \mathbf{i}_{n}^{\mathrm{e}}, 0, \ldots\right)$, $\left(v_{0}, \ldots, v_{n-1}, v_{n}, 0, \ldots\right)$ satisfy equations (7.5.1), (7.5.2) for $m \leqslant n$. The construction of $\mathbf{i}^{\mathrm{e}}, v$ goes on inductively.

7.6 Definition. A unit transformation of an $A_{\infty}$-category $\mathcal{C}$ is a natural $A_{\infty}$-transformation $\mathbf{i}^{\mathrm{e}}:$ ide $\rightarrow$ ide $: \mathcal{C} \rightarrow \mathcal{C}$ such that $\left(\mathbf{i}^{\mathcal{C}} \otimes \mathbf{i}^{\mathrm{e}}\right) B_{2} \equiv \mathbf{i}^{\mathrm{e}}$, and for each pair $X$, $Y$ of objects of $\mathcal{C}$ the chain maps $\left(1 \otimes_{Y} \mathbf{i}_{0}^{\mathcal{C}}\right) b_{2},\left({ }_{X} \mathbf{i}_{0}^{\mathfrak{e}} \otimes 1\right) b_{2}: s \mathcal{C}(X, Y) \rightarrow s \mathcal{C}(X, Y)$ are homotopy invertible. 
We have shown in Proposition 7.5 that an $A_{\infty}$-category $\mathcal{C}$ is unital if and only if it has a unit transformation. Similar (although not identical to our) definitions of units and unital $A_{\infty}$-categories are proposed by Kontsevich and Soibelman [KS02] and Lefèvre-Hasegawa [LH02].

7.7 Proposition. Let $\mathcal{A}, \mathcal{C}$ be $A_{\infty}$-categories. If $\mathcal{C}$ is unital, then $A_{\infty}(\mathcal{A}, \mathcal{C})$ is unital as well.

Proof. We claim that

$$
\left(1 \otimes \mathbf{i}^{\mathfrak{e}}\right) M:\left(1 \otimes \operatorname{id}_{\mathcal{C}}\right) M \rightarrow\left(1 \otimes \operatorname{id}_{\mathcal{C}}\right) M: A_{\infty}(\mathcal{A}, \mathcal{C}) \rightarrow A_{\infty}(\mathcal{A}, \mathcal{C})
$$

is a unit of $A_{\infty}(\mathcal{A}, \mathcal{C})$. Indeed, $\left(1 \otimes \operatorname{id}_{\mathcal{C}}\right) M=\operatorname{id}_{A_{\infty}(\mathcal{A}, \mathcal{C})}$, and there is a 3 -morphism $v:\left(\mathbf{i}^{\mathfrak{e}} \otimes \mathbf{i}^{\mathfrak{e}}\right) B_{2} \rightarrow \mathbf{i}^{\mathrm{e}}: \operatorname{id}_{\mathcal{e}} \rightarrow \operatorname{id}_{\mathcal{C}}: \mathcal{C} \rightarrow \mathcal{C}$. Hence, $\left(\mathbf{i}^{\mathfrak{e}} \otimes \mathbf{i}^{\mathfrak{e}}\right) B_{2}=\mathbf{i}^{\mathfrak{e}}+v B_{1}$, and by

$$
\begin{aligned}
& {\left[\left(1 \otimes \mathbf{i}^{\mathrm{e}}\right) M \otimes\left(1 \otimes \mathbf{i}^{\mathrm{e}}\right) M\right] \tilde{B}_{2}=\left[1 \otimes\left(\mathbf{i}^{\mathrm{e}} \otimes \mathbf{i}^{\mathrm{e}}\right) B_{2}\right] M} \\
& =\left(1 \otimes \mathbf{i}^{\mathrm{e}}\right) M+\left(1 \otimes v B_{1}\right) M=\left(1 \otimes \mathbf{i}^{\mathrm{e}}\right) M+(1 \otimes v) M \tilde{B}_{1} \equiv\left(1 \otimes \mathbf{i}^{\mathrm{e}}\right) M .
\end{aligned}
$$

Let $f: \mathcal{A} \rightarrow \mathcal{C}$ be an $A_{\infty}$-functor. The 0 -th component of $\left(1 \otimes \mathbf{i}^{\mathfrak{C}}\right) M$ is ${ }_{f}[(1 \otimes$ $\left.\left.\mathbf{i}^{\mathrm{e}}\right) M\right]_{0}: \mathbb{k} \rightarrow s A_{\infty}(\mathcal{A}, \mathcal{C})(f, f), 1 \mapsto f \mathbf{i}^{\mathrm{e}}$. It remains to prove that for each pair of $A_{\infty}$-functors $f, g: \mathcal{A} \rightarrow \mathcal{C}$ the maps

$$
\begin{array}{ll}
\left(1 \otimes{ }_{g}\left[\left(1 \otimes \mathbf{i}^{\mathfrak{e}}\right) M\right]_{0}\right) B_{2}: s A_{\infty}(\mathcal{A}, \mathcal{C})(f, g) \rightarrow s A_{\infty}(\mathcal{A}, \mathcal{C})(f, g), & r \mapsto\left(r \otimes g \mathbf{i}^{\mathfrak{e}}\right) B_{2}, \\
\left({ }_{f}\left[\left(1 \otimes \mathbf{i}^{\mathrm{e}}\right) M\right]_{0} \otimes 1\right) B_{2}: s A_{\infty}(\mathcal{A}, \mathcal{C})(f, g) \rightarrow s A_{\infty}(\mathcal{A}, \mathcal{C})(f, g), & r \mapsto r\left(f \mathbf{i}^{\mathfrak{C}} \otimes 1\right) B_{2},
\end{array}
$$

are homotopy invertible.

Let us define a decreasing filtration of the complex $\left(s A_{\infty}(\mathcal{A}, \mathcal{C})(f, g), B_{1}\right)$. For $n \in \mathbb{Z}_{\geqslant 0}$, we set

$$
\begin{aligned}
\Phi_{n} & =\left\{r \in s A_{\infty}(\mathcal{A}, \mathcal{C})(f, g) \mid \forall l<n \quad r_{l}=0\right\} \\
& =\left\{r \in s A_{\infty}(\mathcal{A}, \mathcal{C})(f, g) \mid \forall l<n \quad\left(T^{l} s \mathcal{A}\right) r=0\right\} .
\end{aligned}
$$

Clearly, $\Phi_{n}$ is stable under $B_{1}=[-, b]$, and we have

$$
s A_{\infty}(\mathcal{A}, \mathcal{C})(f, g)=\Phi_{0} \supset \Phi_{1} \supset \cdots \supset \Phi_{n} \supset \Phi_{n+1} \supset \ldots \quad .
$$

Due to (5.1.3) and (3.0.1) the chain maps $a=\left(1 \otimes g \mathbf{i}^{\mathbf{e}}\right) B_{2}, c=\left(f \mathbf{i}^{\mathrm{e}} \otimes 1\right) B_{2}$ preserve the subcomplex $\Phi_{n}$. By Definition $2.6 s A_{\infty}(\mathcal{A}, \mathcal{C})(f, g)=\prod_{n=0}^{\infty} V_{n}$, where $V_{n}$ is the $\mathbb{k}$-module (2.7.1) of $n$-th components $r_{n}$ of $(f, g)$-coderivations $r$. The filtration consists of $\mathbb{k}$-submodules $\Phi_{n}=0 \times \cdots \times 0 \times \prod_{m=n}^{\infty} V_{m}$.

The graded complex associated with this filtration is $\oplus_{n=0}^{\infty} V_{n}$, and the differential $d: V_{n} \rightarrow V_{n}$ induced by $B_{1}$ is given by formula (2.7.2). The associated endomorphisms gr $a$, gr $c$ of $\oplus_{n=0}^{\infty} V_{n}$ are given by the formulas

$$
\begin{gathered}
\left(r_{n}\right) \operatorname{gr} a=\left(r_{n} \otimes{ }_{g} \mathbf{i}_{0}^{\mathbf{e}}\right) b_{2}=\prod_{X_{0}, \ldots, X_{n} \in \mathrm{Ob} \mathcal{A}}\left(x_{0}, \ldots, X_{n} r_{n} \otimes X_{n} \mathbf{i}_{0}^{\mathbf{e}}\right) b_{2}, \\
\left(r_{n}\right) \operatorname{gr} c=r_{n}\left({ }_{f} \mathbf{i}_{0}^{\mathrm{e}} \otimes 1\right) b_{2}=\prod_{X_{0}, \ldots, X_{n} \in \mathrm{Ob} \mathcal{A}} X_{0}, \ldots, X_{n} r_{n}\left(X_{0} f \mathbf{i}_{0}^{\mathrm{e}} \otimes 1\right) b_{2},
\end{gathered}
$$


$r_{n} \in V_{n}$, as formulas (3.0.1), (5.1.3) show. Due to Lemma 7.4 for each pair $X, Y$ of objects of $\mathcal{C}$ the chain maps $\left(1 \otimes_{Y} \mathbf{i}_{0}^{\mathrm{e}}\right) b_{2},-\left({ }_{X} \mathbf{i}_{0}^{\mathrm{e}} \otimes 1\right) b_{2}: s \mathfrak{C}(X, Y) \rightarrow s \mathcal{C}(X, Y)$ are homotopic to the identity map, that is, $\left(1 \otimes_{Y} \mathbf{i}_{0}^{\mathrm{C}}\right) b_{2}=1+h d+d h,\left(X_{0} \mathbf{i}_{0}^{\mathrm{e}} \otimes 1\right) b_{2}=$ $-1+h^{\prime} d+d h^{\prime}$ for some $\mathbb{k}$-linear maps $h, h^{\prime}: s \mathcal{C}(X, Y) \rightarrow s \mathcal{C}(X, Y)$ of degree -1 . Let us choose such homotopies $X_{0}, X_{n} h, X_{0}, X_{n} h^{\prime}: s \mathcal{C}\left(X_{0} f, X_{n} g\right) \rightarrow s \mathfrak{C}\left(X_{0} f, X_{n} g\right)$ for each pair $X_{0}, X_{n}$ of objects of $\mathcal{A}$. Denote by $H, H^{\prime}: \prod_{n=0}^{\infty} V_{n} \rightarrow \prod_{n=0}^{\infty} V_{n}$ the diagonal maps $X_{0}, \ldots, X_{n} r_{n} \mapsto X_{0}, \ldots, X_{n} r_{n X_{0}, X_{n}} h, X_{0}, \ldots, X_{n} r_{n} \mapsto X_{0}, \ldots, X_{n} r_{n X_{0}, X_{n}} h^{\prime}$. Then gr $a=1+H d+d H$, gr $c=-1+H^{\prime} d+d H^{\prime}$. The chain maps $a-H B_{1}-B_{1} H, c-$ $H^{\prime} B_{1}-B_{1} H^{\prime}$, being restricted to maps $\oplus_{m=0}^{\infty} V_{m} \rightarrow \prod_{m=0}^{\infty} V_{m}$ give upper triangular $\mathbb{N} \times \mathbb{N}$ matrices which, in turn, determine the whole map. Thus, $a-H B_{1}-B_{1} H=$ $1+N, c-H^{\prime} B_{1}-B_{1} H^{\prime}=-1+N^{\prime}$, where the $\mathbb{N} \times \mathbb{N}$ matrices $N, N^{\prime}$ are strictly upper triangular. Therefore, $1+N$ and $-1+N^{\prime}$ are invertible (since their inverse maps $\sum_{i=0}^{\infty}(-N)^{i}$ and $-\sum_{i=0}^{\infty}\left(N^{\prime}\right)^{i}$ make sense). Hence, $a=\left(1 \otimes g \mathbf{i}^{\mathfrak{e}}\right) B_{2}$ and $c=\left(f \mathbf{i}^{\mathfrak{e}} \otimes 1\right) B_{2}$ are invertible in $\mathcal{K}$.

7.8 Corollary. Let $f, g: \mathcal{A} \rightarrow \mathcal{C}$ be $A_{\infty}$-functors. If $\mathcal{C}$ is unital, then

$$
\begin{aligned}
\left(1 \otimes g \mathbf{i}^{\mathrm{C}}\right) B_{2} & \sim 1: s A_{\infty}(\mathcal{A}, \mathcal{C})(f, g) \rightarrow s A_{\infty}(\mathcal{A}, \mathcal{C})(f, g), \text { and } \\
\left(f \mathbf{i}^{\mathcal{C}} \otimes 1\right) B_{2} \sim-1: s A_{\infty}(\mathcal{A}, \mathcal{C})(f, g) & \rightarrow s A_{\infty}(\mathcal{A}, \mathcal{C})(f, g) .
\end{aligned}
$$

Proof. Follows from Proposition 7.7 and Lemma 7.4.

7.9 Corollary. Let $r: f \rightarrow g: \mathcal{A} \rightarrow \mathcal{C}$ be a natural $A_{\infty}$-transformation. If $\mathcal{C}$ is unital, then

$$
\left(r \otimes g \mathbf{i}^{\mathrm{e}}\right) B_{2} \equiv r, \quad\left(f \mathbf{i}^{\mathrm{e}} \otimes r\right) B_{2} \equiv r .
$$

Proof. By Corollary 7.8 there are homotopies $h, h^{\prime}: s A_{\infty}(\mathcal{A}, \mathcal{C})(f, g) \rightarrow$ $s A_{\infty}(\mathcal{A}, \mathrm{C})(f, g)$, which give

$$
\begin{gathered}
\left(r \otimes g \mathbf{i}^{\mathrm{e}}\right) B_{2}=r\left(1 \otimes g \mathbf{i}^{\mathrm{e}}\right) B_{2}=r+r B_{1} h+r h B_{1}=r+(r h) B_{1} \equiv r, \\
\left(f \mathbf{i}^{\mathrm{C}} \otimes r\right) B_{2}=-r\left(f \mathbf{i}^{\mathrm{e}} \otimes 1\right) B_{2}=r+r B_{1} h^{\prime}+r h^{\prime} B_{1}=r+\left(r h^{\prime}\right) B_{1} \equiv r .
\end{gathered}
$$

7.10 Corollary. The unit transformation of a unital category is determined uniquely up to equivalence.

Indeed, take $f=$ ide and notice that any two unit transformations $\mathbf{i}^{\mathrm{e}}$ and ${ }^{\prime} \mathbf{i}^{\mathrm{e}}$ of C satisfy ${ }^{\prime} \mathbf{i}^{\mathrm{e}} \equiv{ }^{\prime} \mathbf{i}^{\mathrm{e}} \cdot \mathbf{i}^{\mathrm{e}} \equiv \mathbf{i}^{\mathrm{e}}$.

7.11 Corollary. The full $\mathcal{K}$-2-subcategory $\mathcal{K}^{u} A_{\infty}$ of non-2-unital $\mathcal{K}$-2-category $\mathcal{K} A_{\infty}$, whose objects are unital $A_{\infty}$-categories and the other data are as in $\mathcal{K} A_{\infty}$, is a 1-2-unital $\mathcal{K}$-2-category. The unit 2-endomorphism of an $A_{\infty}$-functor $f: \mathcal{A} \rightarrow \mathcal{C}$ is the homotopy class of the chain map

$$
1_{f}: \mathbb{k} \rightarrow\left(A_{\infty}(\mathcal{A}, \mathcal{C})(f, f), m_{1}\right), \quad 1 \mapsto\left(f \mathbf{i}^{\mathrm{C}}\right) s^{-1} .
$$

Proof. The composition

$$
\begin{gathered}
A_{\infty}(\mathcal{A}, \mathcal{C})(f, g) \stackrel{1 \otimes 1_{g}}{\longrightarrow} A_{\infty}(\mathcal{A}, \mathcal{C})(f, g) \otimes A_{\infty}(\mathcal{A}, \mathcal{C})(g, g) \stackrel{m_{2}}{\longrightarrow} A_{\infty}(\mathcal{A}, \mathcal{C})(f, g), \\
r s^{-1} \mapsto r s^{-1} \otimes\left(g \mathbf{i}^{\mathrm{C}}\right) s^{-1} \mapsto\left(r s^{-1} \otimes\left(g \mathbf{i}^{\mathrm{e}}\right) s^{-1}\right)(s \otimes s) B_{2} s^{-1}=\left(r \otimes g \mathbf{i}^{\mathrm{C}}\right) B_{2} s^{-1},
\end{gathered}
$$


is homotopic to the identity map by Corollary 7.8. Similarly, the composition

$$
\begin{gathered}
A_{\infty}(\mathcal{A}, \mathcal{C})(f, g) \stackrel{1_{f} \otimes 1}{\longrightarrow} A_{\infty}(\mathcal{A}, \mathcal{C})(f, f) \otimes A_{\infty}(\mathcal{A}, \mathcal{C})(f, g) \stackrel{m_{2}}{\longrightarrow} A_{\infty}(\mathcal{A}, \mathcal{C})(f, g), \\
r s^{-1} \mapsto\left(f \mathbf{i}^{\mathcal{C}}\right) s^{-1} \otimes r s^{-1} \mapsto(-)^{r-1}\left(f \mathbf{i}^{\mathcal{C}} \otimes r\right) B_{2} s^{-1}=-r\left(f \mathbf{i}^{\mathcal{C}} \otimes 1\right) B_{2} s^{-1},
\end{gathered}
$$

is homotopic to the identity map.

7.12 Corollary. The full 2-subcategory ${ }^{u} A_{\infty}$ of non-2-unital 2-category $A_{\infty}$, which consists of unital $A_{\infty}$-categories, all $A_{\infty}$-functors between them, and equivalence classes of all natural $A_{\infty}$-transformations is a (usual 1-2-unital) 2-category. The unit 2-endomorphism of an $A_{\infty}$-functor $f: \mathcal{A} \rightarrow \mathcal{C}$ is $f \mathbf{i}^{\mathrm{C}}$. In this 2-category the notions of an isomorphism between $A_{\infty}$-functors, an equivalence between $A_{\infty}$-categories, etc. make sense. For instance, $r: f \rightarrow g: \mathcal{A} \rightarrow \mathcal{B}$ is an isomorphism if there is a natural $A_{\infty}$-transformation $p: g \rightarrow f$, such that $(r \otimes p) B_{2} \equiv f \mathbf{i}^{\mathcal{B}}$ and $(p \otimes r) B_{2} \equiv g \mathbf{i}^{\mathcal{B}}$. An $A_{\infty}$-functor $f: \mathcal{A} \rightarrow \mathcal{B}$ is an equivalence if there exists an $A_{\infty}$-functor $g: \mathcal{B} \rightarrow \mathcal{A}$ and isomorphisms $\mathrm{id}_{\mathcal{A}} \rightarrow f g$ and $\mathrm{id}_{\mathcal{B}} \rightarrow g f$.

Proof. Follows from Corollary 7.9 or 7.11 .

7.13. Invertible transformations. Let $\mathcal{B}, \mathcal{C}$ be $A_{\infty}$-categories, and let $f, g$ : $\mathrm{ObC} \rightarrow \mathrm{ObB}$ be maps. Assume that $\mathcal{B}$ is unital and that for each object $X$ of $\mathrm{C}$ there are $\mathbb{k}$-linear maps

$$
\begin{array}{cl}
X r_{0}: \mathbb{k} \rightarrow(s \mathcal{B})^{-1}(X f, X g), & X p_{0}: \mathbb{k} \rightarrow(s \mathcal{B})^{-1}(X g, X f), \\
X w_{0}: \mathbb{k} \rightarrow(s \mathcal{B})^{-2}(X f, X f), & X v_{0}: \mathbb{k} \rightarrow(s \mathcal{B})^{-2}(X g, X g),
\end{array}
$$

such that

$$
\begin{gathered}
{ }_{X} r_{0} b_{1}=0, \quad{ }_{X} p_{0} b_{1}=0, \\
\left({ }_{X} r_{0} \otimes_{X} p_{0}\right) b_{2}-{ }_{X} \mathbf{i}_{0}^{\mathcal{B}}={ }_{X} w_{0} b_{1}, \\
\left({ }_{X} p_{0} \otimes_{X} r_{0}\right) b_{2}-{ }_{X g} \mathbf{i}_{0}^{\mathcal{B}}={ }_{X} v_{0} b_{1} .
\end{gathered}
$$

7.14 Lemma. Let the above assumptions hold. Then for all objects $X$ of $\mathcal{C}$ and $Y$ of $\mathcal{B}$ the chain maps

$$
\begin{aligned}
& \left(r_{0} \otimes 1\right) b_{2}: s \mathcal{B}(X g, Y) \rightarrow s \mathcal{B}(X f, Y) \text { and }\left(p_{0} \otimes 1\right) b_{2}: s \mathcal{B}(X f, Y) \rightarrow s \mathcal{B}(X g, Y), \\
& \left(1 \otimes r_{0}\right) b_{2}: s \mathcal{B}(Y, X f) \rightarrow s \mathcal{B}(Y, X g) \text { and }\left(1 \otimes p_{0}\right) b_{2}: s \mathcal{B}(Y, X g) \rightarrow s \mathcal{B}(Y, X f)
\end{aligned}
$$

are homotopy inverse to each other.

Proof. We have

$$
\begin{aligned}
& \left(r_{0} \otimes 1\right) b_{2}\left(p_{0} \otimes 1\right) b_{2}=\left(p_{0} \otimes r_{0} \otimes 1\right)\left(1 \otimes b_{2}\right) b_{2} \\
& =-\left(p_{0} \otimes r_{0} \otimes 1\right)\left[\left(b_{2} \otimes 1\right) b_{2}+b_{3} b_{1}+\left(1 \otimes 1 \otimes b_{1}\right) b_{3}\right] \\
& =-\left(X g \mathbf{i}_{0}^{\mathcal{B}} \otimes 1\right) b_{2}-\left(v_{0} b_{1} \otimes 1\right) b_{2}-\left(p_{0} \otimes r_{0} \otimes 1\right) b_{3} b_{1}-b_{1}\left(p_{0} \otimes r_{0} \otimes 1\right) b_{3} \\
& \sim 1+b_{1}\left(v_{0} \otimes 1\right) b_{2}+\left(v_{0} \otimes 1\right) b_{2} b_{1} \sim 1: s \mathcal{B}(X g, Y) \rightarrow s \mathcal{B}(X g, Y) .
\end{aligned}
$$

For symmetry reasons also

$$
\left(p_{0} \otimes 1\right) b_{2}\left(r_{0} \otimes 1\right) b_{2} \sim 1: s \mathcal{B}(X f, Y) \rightarrow s \mathcal{B}(X f, Y) .
$$


Therefore, $\left(r_{0} \otimes 1\right) b_{2}$ and $\left(p_{0} \otimes 1\right) b_{2}$ are homotopy inverse to each other.

Similarly, $\left(1 \otimes r_{0}\right) b_{2}$ and $\left(1 \otimes p_{0}\right) b_{2}$ are homotopy inverse to each other.

7.15 Proposition. Let $r: f \rightarrow g: \mathcal{C} \rightarrow \mathcal{B}$ be a natural $A_{\infty}$-transformation, where $\mathcal{B}$ is unital, and let $p_{0}, v_{0}, w_{0}$ be as in Section 7.13 so that equations (7.13.1) hold. Then $p_{0}, w_{0}$ extend to a natural $A_{\infty}$-transformation $p: g \rightarrow f: \mathcal{C} \rightarrow \mathcal{B}$, a 3-morphism $w$, and there is a 3-morphism $t$ as follows:

$$
\begin{aligned}
w:(r \otimes p) B_{2} & \rightarrow f \mathbf{i}^{\mathcal{B}}: f \rightarrow f: \mathcal{C} \rightarrow \mathcal{B}, \\
t:(p \otimes r) B_{2} & \rightarrow g \mathbf{i}^{\mathcal{B}}: g \rightarrow g: \mathcal{C} \rightarrow \mathcal{B} .
\end{aligned}
$$

In particular, $r$ is invertible and $p=r^{-1}$ in $A_{\infty}$.

Proof. Let us drop equation (7.15.2) and prove the existence of $p$ and $w$, satisfying (7.15.1). We have to satisfy the equations

$$
\begin{aligned}
p b+b p & =0, \\
(r \otimes p) B_{2}-f \mathbf{i}^{\mathcal{B}} & =[w, b] .
\end{aligned}
$$

Let us construct the components of $p$ and $w$ by induction. Given a positive $n$, assume that we have already found components $p_{m}, w_{m}$ of the sought $p, w$ for $m<n$, such that the equations

$$
\begin{gathered}
(p b)_{m}+(b p)_{m}=0: s \mathcal{C}\left(X_{0}, X_{1}\right) \otimes \cdots \otimes s \mathcal{C}\left(X_{m-1}, X_{m}\right) \rightarrow s \mathcal{B}\left(X_{0} g, X_{m} f\right), \\
{\left[(r \otimes p) B_{2}\right]_{m}-\left(f \mathbf{i}^{\mathcal{B}}\right)_{m}=(w b)_{m}-(b w)_{m}:} \\
s \mathcal{C}\left(X_{0}, X_{1}\right) \otimes \cdots \otimes s \mathcal{C}\left(X_{m-1}, X_{m}\right) \rightarrow s \mathcal{B}\left(X_{0} f, X_{m} f\right)
\end{gathered}
$$

are satisfied for all $m<n$. Introduce a $(g, f)$-coderivation $\tilde{p}: T s \mathcal{C} \rightarrow T s \mathcal{B}$ of degree -1 by its components $\left(p_{0}, p_{1}, \ldots, p_{n-1}, 0,0, \ldots\right)$ and an $(f, f)$-coderivation $\tilde{w}: T s \mathcal{C} \rightarrow T s \mathcal{B}$ of degree -2 by its components $\left(w_{0}, w_{1}, \ldots, w_{n-1}, 0,0, \ldots\right)$. Define a $(g, f)$-coderivation $\lambda=\tilde{p} b+b \tilde{p}$ of degree 0 and an $(f, f)$-coderivation $\nu=(r \otimes$ $\tilde{p}) B_{2}-f \mathbf{i}^{\mathcal{B}}-[\tilde{w}, b]$ of degree -1 . Then equations (7.15.3), (7.15.4) imply that $\lambda_{m}=0$, $\nu_{m}=0$ for $m<n$. The identity $\lambda b-b \lambda=0$ implies that

$$
\lambda_{n} d=\lambda_{n} b_{1}-\sum_{q+1+t=n}\left(1^{\otimes q} \otimes b_{1} \otimes 1^{\otimes t}\right) \lambda_{n}=0 .
$$

The identity

$$
\begin{array}{r}
{[\nu, b]=\nu B_{1}=(r \otimes \tilde{p}) B_{2} B_{1}-\left(f \mathbf{i}^{\mathcal{B}}\right) B_{1}-\tilde{w} B_{1} B_{1}=-(r \otimes \tilde{p})\left(1 \otimes B_{1}+B_{1} \otimes 1\right) B_{2}} \\
=-\left(r \otimes \tilde{p} B_{1}\right) B_{2}=-(r \otimes \lambda) B_{2}
\end{array}
$$

implies that

$$
\nu_{n} b_{1}+\sum_{q+1+t=n}\left(1^{\otimes q} \otimes b_{1} \otimes 1^{\otimes t}\right) \nu_{n}=-\left(r_{0} \otimes \lambda_{n}\right) b_{2},
$$

that is, $\nu_{n} d=-\lambda_{n} u$. Here the map $u=\operatorname{Hom}\left(N,\left(r_{0} \otimes 1\right) b_{2}\right)$ for $N=s \mathfrak{C}\left(X_{0}, X_{1}\right) \otimes_{\mathbb{k}}$ $\cdots \otimes_{\mathbb{k}} s \mathfrak{C}\left(X_{n-1}, X_{n}\right)$ is homotopy invertible, and the complex Cone $(u)$ is contractible by Lemma Appendix B.1. Hence, the cycle

$$
\left(\nu_{n}, \lambda_{n}\right) \in \operatorname{Hom}_{\mathbb{k}}^{-1}\left(N, s \mathcal{B}\left(X_{0} f, X_{n} f\right)\right) \oplus \operatorname{Hom}_{\mathbb{k}}^{0}\left(N, s \mathcal{B}\left(X_{0} g, X_{n} f\right)\right)=\operatorname{Cone}^{-1}(u)
$$


is the boundary of some element

$$
\left(w_{n}, p_{n}\right) \in \operatorname{Hom}_{\mathbb{k}}^{-2}\left(N, s \mathcal{B}\left(X_{0} f, X_{n} f\right)\right) \oplus \operatorname{Hom}_{\mathbb{k}}^{-1}\left(N, s \mathcal{B}\left(X_{0} g, X_{n} f\right)\right)=\operatorname{Cone}^{-2}(u),
$$

that is, $w_{n} d+p_{n} u=\nu_{n}$ and $-p_{n} d=\lambda_{n}$. In other words, equations (7.15.5), (7.15.6) are satisfied for $m=n$, and we prove the statement by induction.

For similar reasons using Lemma 7.14 there exists a natural $A_{\infty}$-transformation $q: g \rightarrow f: \mathcal{C} \rightarrow \mathcal{B}$ with $q_{0}=p_{0}$ and a 3-morphism

$$
v:(q \otimes r) B_{2} \rightarrow g \mathbf{i}^{\mathcal{B}}: g \rightarrow g: \mathcal{C} \rightarrow \mathcal{B}
$$

with given $v_{0}$. Since $r$ has a left inverse and a right inverse, it is invertible in $A_{\infty}$ and $p$ is equivalent to $q$. Hence, $(p \otimes r) B_{2}$ is equivalent to $(q \otimes r) B_{2}$, and there exists $t$ of (7.15.2).

\section{Unital $A_{\infty}$-functors}

8.1 Definition. Let $\mathcal{A}, \mathcal{B}$ be unital $A_{\infty}$-categories. An $A_{\infty}$-functor $f: \mathcal{A} \rightarrow \mathcal{B}$ is called unital if for all objects $X$ of $\mathcal{A}$ we have ${ }_{X} \mathbf{i}_{0}^{\mathcal{A}} f_{1}-{ }_{X f} \mathbf{i}_{0}^{\mathcal{B}} \in \operatorname{Im} b_{1}$.

For instance, an $A_{\infty}$-homomorphism $f: \mathcal{A} \rightarrow \mathcal{B}$ of $A_{\infty}$-algebras is unital if the cycles $\mathbf{i}_{0}^{\mathcal{A}} f_{1}, \mathbf{i}_{0}^{\mathcal{B}} \in(s \mathcal{B})^{-1}$ are cohomologous in $\left(s \mathcal{B}, b_{1}\right)$. We may say that a unital $A_{\infty}$-functor (or $A_{\infty}$-homomorphism) preserves the cohomology classes of unit elements.

8.2 Proposition. Let $\mathcal{A}, \mathcal{B}$ be unital $A_{\infty}$-categories. An $A_{\infty}$-functor $f: \mathcal{A} \rightarrow \mathcal{B}$ is unital if and only if $\mathbf{i}^{\mathcal{A}} f \equiv f \mathbf{i}^{\mathcal{B}}$.

Proof. If $\mathbf{i}^{\mathcal{A}} f=f \mathbf{i}^{\mathcal{B}}+v B_{1}$, then ${ }_{X} \mathbf{i}_{0}^{\mathcal{A}} f_{1}={ }_{X}\left(\mathbf{i}^{\mathcal{A}} f\right)_{0}={ }_{X}\left(f \mathbf{i}^{\mathcal{B}}+v B_{1}\right)_{0}={ }_{X f} \mathbf{i}_{0}^{\mathcal{B}}+$ ${ }_{X} v_{0} b_{1}$, hence, $f$ is unital.

Assume now that $f$ is unital. We want to find a 3-morphism

$$
v: \mathbf{i}^{\mathcal{A}} f \rightarrow f \mathbf{i}^{\mathcal{B}}: f \rightarrow f: \mathcal{A} \rightarrow \mathcal{B}
$$

that is, an $(f, f)$-coderivation $v$ of degree -2 such that

$$
v B_{1}=\mathbf{i}^{\mathcal{A}} f-f \mathbf{i}^{\mathcal{B}} \text {. }
$$

We subject it to an additional condition described below. Consider 3-morphisms

$$
\begin{aligned}
& x:\left(\mathbf{i}^{\mathcal{A}} \otimes \mathbf{i}^{\mathcal{A}}\right) B_{2} \rightarrow \mathbf{i}^{\mathcal{A}}: \operatorname{id}_{\mathcal{A}} \rightarrow \mathrm{id}_{\mathcal{A}}: \mathcal{A} \rightarrow \mathcal{A}, \\
& y:\left(\mathbf{i}^{\mathcal{B}} \otimes \mathbf{i}^{\mathcal{B}}\right) B_{2} \rightarrow \mathbf{i}^{\mathcal{B}}: \mathrm{id}_{\mathcal{B}} \rightarrow \mathrm{id}_{\mathcal{B}}: \mathcal{B} \rightarrow \mathcal{B},
\end{aligned}
$$

so that

$$
x B_{1}=\left(\mathbf{i}^{\mathcal{A}} \otimes \mathbf{i}^{\mathcal{A}}\right) B_{2}-\mathbf{i}^{\mathcal{A}}, \quad y B_{1}=\left(\mathbf{i}^{\mathcal{B}} \otimes \mathbf{i}^{\mathcal{B}}\right) B_{2}-\mathbf{i}^{\mathcal{B}} .
$$

The following equations between $(f, f)$-coderivations of degree -2 are due to (7.1.2), (7.1.3):

$$
\begin{gathered}
(x f) B_{1}=\left(\mathbf{i}^{\mathcal{A}} \otimes \mathbf{i}^{\mathcal{A}}\right) B_{2} f-\mathbf{i}^{\mathcal{A}} f=\left(\mathbf{i}^{\mathcal{A}} f \otimes \mathbf{i}^{\mathcal{A}} f\right) B_{2}+\left(\mathbf{i}^{\mathcal{A}} \otimes \mathbf{i}^{\mathcal{A}} \mid f\right) M_{20} B_{1}-\mathbf{i}^{\mathcal{A}} f \\
(f y) B_{1}=f\left(\mathbf{i}^{\mathcal{B}} \otimes \mathbf{i}^{\mathcal{B}}\right) B_{2}-f \mathbf{i}^{\mathcal{B}}=\left(f \mathbf{i}^{\mathcal{B}} \otimes f \mathbf{i}^{\mathcal{B}}\right) B_{2}-f \mathbf{i}^{\mathcal{B}}
\end{gathered}
$$


Combining them with (8.2.1) we find that

$$
\begin{aligned}
& (x f) B_{1}-\left(\mathbf{i}^{\mathcal{A}} \otimes \mathbf{i}^{\mathcal{A}} \mid f\right) M_{20} B_{1}+v B_{1} \\
& =\left(\mathbf{i}^{\mathcal{A}} f \otimes \mathbf{i}^{\mathcal{A}} f\right) B_{2}-f \mathbf{i}^{\mathcal{B}} \\
& =\left(\mathbf{i}^{\mathcal{A}} f \otimes v B_{1}\right) B_{2}+\left(\mathbf{i}^{\mathcal{A}} f \otimes f \mathbf{i}^{\mathcal{B}}\right) B_{2}-f \mathbf{i}^{\mathcal{B}} \\
& =-\left(\mathbf{i}^{\mathcal{A}} f \otimes v\right) B_{2} B_{1}+\left(v B_{1} \otimes f \mathbf{i}^{\mathcal{B}}\right) B_{2}+\left(f \mathbf{i}^{\mathcal{B}} \otimes f \mathbf{i}^{\mathcal{B}}\right) B_{2}-f \mathbf{i}^{\mathcal{B}} \\
& =-\left(\mathbf{i}^{\mathcal{A}} f \otimes v\right) B_{2} B_{1}+\left(v \otimes f \mathbf{i}^{\mathcal{B}}\right) B_{2} B_{1}+(f y) B_{1} .
\end{aligned}
$$

Now we may formulate the problem: we are looking for $v$ as above and an $(f, f)$ coderivation $w$ of degree -3 , such that

$$
w B_{1}=x f-\left(\mathbf{i}^{\mathcal{A}} \otimes \mathbf{i}^{\mathcal{A}} \mid f\right) M_{20}+v+\left(\mathbf{i}^{\mathcal{A}} f \otimes v\right) B_{2}-\left(v \otimes f \mathbf{i}^{\mathcal{B}}\right) B_{2}-f y,
$$

in other terms, a 4-morphism

$$
\begin{aligned}
w: x f-\left(\mathbf{i}^{\mathcal{A}} \otimes \mathbf{i}^{\mathcal{A}} \mid f\right) M_{20}+v \rightarrow f y- & \left(\mathbf{i}^{\mathcal{A}} f \otimes v\right) B_{2}+\left(v \otimes f \mathbf{i}^{\mathcal{B}}\right) B_{2}: \\
& \left(\mathbf{i}^{\mathcal{A}} f \otimes \mathbf{i}^{\mathcal{A}} f\right) B_{2} \rightarrow f \mathbf{i}^{\mathcal{B}}: f \rightarrow f: \mathcal{A} \rightarrow \mathcal{B} .
\end{aligned}
$$

Using the chain map

$u=\left(1 \otimes f \mathbf{i}^{\mathcal{B}}\right) B_{2}-1-\left(\mathbf{i}^{\mathcal{A}} f \otimes 1\right) B_{2}:\left(s A_{\infty}(\mathcal{A}, \mathcal{B})(f, f), B_{1}\right) \rightarrow\left(s A_{\infty}(\mathcal{A}, \mathcal{B})(f, f), B_{1}\right)$, we may rewrite our system of equations as follows:

$$
\begin{aligned}
-v B_{1} & =f \mathbf{i}^{\mathcal{B}}-\mathbf{i}^{\mathcal{A}} f, \\
w B_{1}+v u & =x f-\left(\mathbf{i}^{\mathcal{A}} \otimes \mathbf{i}^{\mathcal{A}} \mid f\right) M_{20}-f y .
\end{aligned}
$$

In other words, we look for an element

$$
(w, v) \in\left[s A_{\infty}(\mathcal{A}, \mathcal{B})(f, f)\right]^{-3} \oplus\left[s A_{\infty}(\mathcal{A}, \mathcal{B})(f, f)\right]^{-2}=\operatorname{Cone}^{-3}(u),
$$

whose boundary is

$$
\begin{aligned}
\left(x f-\left(\mathbf{i}^{\mathcal{A}} \otimes \mathbf{i}^{\mathcal{A}} \mid f\right)\right. & \left.M_{20}-f y, f \mathbf{i}^{\mathcal{B}}-\mathbf{i}^{\mathcal{A}} f\right) \\
& \in\left[s A_{\infty}(\mathcal{A}, \mathcal{B})(f, f)\right]^{-2} \oplus\left[s A_{\infty}(\mathcal{A}, \mathcal{B})(f, f)\right]^{-1}=\operatorname{Cone}^{-2}(u) .
\end{aligned}
$$

Let us prove that $u$ is homotopy invertible. Since

$$
{ }_{X} \mathbf{i}_{0}^{\mathcal{A}} f_{1}={ }_{X f} \mathbf{i}_{0}^{\mathcal{B}}+{ }_{X} z b_{1}: \mathbb{k} \rightarrow(s \mathcal{B})^{-1}(X f, X f),
$$

for some ${ }_{X} z$, the cycles ${ }_{X} r_{0}={ }_{X} \mathbf{i}_{0}^{\mathcal{A}} f_{1}$ and ${ }_{X} p_{0}={ }_{X f} \mathbf{i}_{0}^{\mathcal{B}}$ satisfy conditions (7.13.1) for $g=f: \mathrm{Ob} \mathcal{A} \rightarrow \mathrm{Ob} \mathcal{B}$, that is,

$$
\begin{aligned}
& \left(x_{i} \mathbf{i}_{0}^{\mathcal{A}} f_{1} \otimes x_{f} \mathbf{i}_{0}^{\mathcal{B}}\right) b_{2}-{ }_{x f} \mathbf{i}_{0}^{\mathcal{B}}=\left(x_{f} \mathbf{i}_{0}^{\mathcal{B}} \otimes x_{f} \mathbf{i}_{0}^{\mathcal{B}}\right) b_{2}-{ }_{X f} \mathbf{i}_{0}^{\mathcal{B}}+\left({ }_{x} z \otimes x_{f} \mathbf{i}_{0}^{\mathcal{B}}\right) b_{2} b_{1} \in \operatorname{Im} b_{1}, \\
& \left(x_{f} \mathbf{i}_{0}^{\mathcal{B}} \otimes x_{X} \mathbf{i}_{0}^{\mathcal{A}} f_{1}\right) b_{2}-x_{x} \mathbf{i}_{0}^{\mathcal{B}}=\left(x_{f} \mathbf{i}_{0}^{\mathcal{B}} \otimes X_{x} \mathbf{i}_{0}^{\mathcal{B}}\right) b_{2}-x_{X f} \mathbf{i}_{0}^{\mathcal{B}}-\left(x_{f} \mathbf{i}_{0}^{\mathcal{B}} \otimes X_{X} z\right) b_{2} b_{1} \in \operatorname{Im} b_{1} .
\end{aligned}
$$

Hence, the natural $A_{\infty}$-transformation $r=\mathbf{i}^{\mathcal{A}} f: f \rightarrow f: \mathcal{A} \rightarrow \mathcal{B}$ is invertible by Proposition 7.15. In detail, there exists a natural $A_{\infty}$-transformation $p: f \rightarrow f:$ $\mathcal{A} \rightarrow \mathcal{B}$ and 3-morphisms $q, t$ such that

$$
\left(\mathbf{i}^{\mathcal{A}} f \otimes p\right) B_{2}-f \mathbf{i}^{\mathcal{B}}=q B_{1}, \quad\left(p \otimes \mathbf{i}^{\mathcal{A}} f\right) B_{2}-f \mathbf{i}^{\mathcal{B}}=t B_{1} .
$$


These equations are interpreted as equations (7.13.1) for the following data. Let $\mathcal{C}=\mathbb{1}$ be a 1-object-0-morphisms $A_{\infty}$-category, Ob $\mathcal{C}=\{*\}, \mathcal{C}(*, *)=0$. Consider a map $\mathrm{Ob} \mathcal{C} \rightarrow \operatorname{Ob} A_{\infty}(\mathcal{A}, \mathcal{B}), * \mapsto f$, and elements $\mathbf{i}^{\mathcal{A}} f, p \in\left[s A_{\infty}(\mathcal{A}, \mathcal{B})(f, f)\right]^{-1}$, $q, t \in\left[s A_{\infty}(\mathcal{A}, \mathcal{B})(f, f)\right]^{-2}$. Equations (7.13.1) for these data are precisely (8.2.3), since ${ }_{f} \mathbf{i}_{0}^{A_{\infty}(\mathcal{A}, \mathcal{B})}=f \mathbf{i}^{\mathcal{B}}$. By Lemma 7.14 we deduce that

$$
\left(\mathbf{i}^{\mathcal{A}} f \otimes 1\right) B_{2}:\left(s A_{\infty}(\mathcal{A}, \mathcal{B})(f, f), B_{1}\right) \rightarrow\left(s A_{\infty}(\mathcal{A}, \mathcal{B})(f, f), B_{1}\right),
$$

is homotopy invertible. Since the map $\left(1 \otimes f \mathbf{i}^{\mathcal{B}}\right) B_{2}-1$ is homotopic to 0 by Corollary 7.8, we deduce that $u$ is homotopy invertible. Therefore, Cone $(u)$ is contractible by Lemma Appendix B.1.

To prove the existence of $(w, v)$ satisfying (8.2.2) it suffices to show that ( $x f-$ $\left.\left(\mathbf{i}^{\mathcal{A}} \otimes \mathbf{i}^{\mathcal{A}} \mid f\right) M_{20}-f y, f \mathbf{i}^{\mathcal{B}}-\mathbf{i}^{\mathcal{A}} f\right) \in \operatorname{Cone}^{-2}(u)$ is a cycle. And indeed,

$$
\begin{aligned}
& {\left[x f-\left(\mathbf{i}^{\mathcal{A}} \otimes \mathbf{i}^{\mathcal{A}} \mid f\right) M_{20}-f y\right] B_{1}+\left(f \mathbf{i}^{\mathcal{B}}-\mathbf{i}^{\mathcal{A}} f\right) u} \\
& =\left(x B_{1}\right) f-\left(\mathbf{i}^{\mathcal{A}} \otimes \mathbf{i}^{\mathcal{A}} \mid f\right) M_{20} B_{1}-f\left(y B_{1}\right) \\
& \quad+\left[\left(f \mathbf{i}^{\mathcal{B}}-\mathbf{i}^{\mathcal{A}} f\right) \otimes f \mathbf{i}^{\mathcal{B}}\right] B_{2}-f \mathbf{i}^{\mathcal{B}}+\mathbf{i}^{\mathcal{A}} f+\left[\mathbf{i}^{\mathcal{A}} f \otimes\left(f \mathbf{i}^{\mathcal{B}}-\mathbf{i}^{\mathcal{A}} f\right)\right] B_{2} \\
& =\left(\mathbf{i}^{\mathcal{A}} \otimes \mathbf{i}^{\mathcal{A}}\right) B_{2} f-\mathbf{i}^{\mathcal{A}} f-\left(\mathbf{i}^{\mathcal{A}} \otimes \mathbf{i}^{\mathcal{A}} \mid f\right) M_{20} B_{1}-f\left(\mathbf{i}^{\mathcal{B}} \otimes \mathbf{i}^{\mathcal{B}}\right) B_{2}+f \mathbf{i}^{\mathcal{B}} \\
& \quad+\left(f \mathbf{i}^{\mathcal{B}} \otimes f \mathbf{i}^{\mathcal{B}}\right) B_{2}-\left(\mathbf{i}^{\mathcal{A}} f \otimes f \mathbf{i}^{\mathcal{B}}\right) B_{2}-f \mathbf{i}^{\mathcal{B}}+\mathbf{i}^{\mathcal{A}} f+\left(\mathbf{i}^{\mathcal{A}} f \otimes f \mathbf{i}^{\mathcal{B}}\right) B_{2} \\
& \quad-\left(\mathbf{i}^{\mathcal{A}} f \otimes \mathbf{i}^{\mathcal{A}} f\right) B_{2} \\
& =-\left[\left(\mathbf{i}^{\mathcal{A}} \otimes \mathbf{i}^{\mathcal{A}}\right)\left(1 \otimes B_{1}+B_{1} \otimes 1\right) \mid f\right] M_{20}=0
\end{aligned}
$$

due to (7.1.2) and (7.1.3). Clearly, $\left(f \mathbf{i}^{\mathcal{B}}-\mathbf{i}^{\mathcal{A}} f\right) B_{1}=0$, so the proposition is proven.

Clearly, the composition of unital functors is unital. If $\mathcal{B}, \mathcal{C}$ are unital $A_{\infty}$-categories, $r: f \rightarrow g: \mathcal{B} \rightarrow \mathcal{C}$ is an isomorphism of $A_{\infty}$-functors and $f$ is unital, then $g$ is unital as well. Indeed, distributivity law in $A_{\infty}$ implies

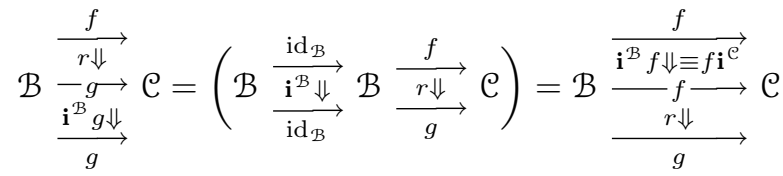

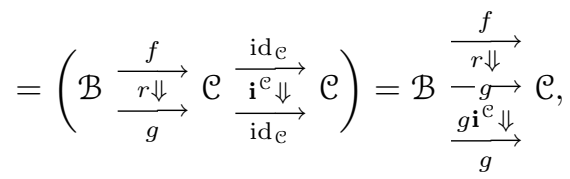

$$
\begin{aligned}
& \text { or } \quad r \cdot\left(\mathbf{i}^{\mathcal{B}} g\right)=\mathbf{i}^{\mathcal{B}} \circ_{h} r=\left(\mathbf{i}^{\mathcal{B}} f\right) \cdot r=\left(f \mathbf{i}^{\mathcal{C}}\right) \cdot r=r \circ_{h} \mathbf{i}^{\mathrm{e}}=r \cdot\left(g \mathbf{i}^{\mathbf{C}}\right),
\end{aligned}
$$

where $\cdot$ and $\circ_{h}$ denote the vertical and the horizontal compositions of 2-morphisms, hence, $\mathbf{i}^{\mathcal{B}} g \equiv g \mathbf{i}^{\mathrm{C}}$.

8.3 Definition. The 2-category $A_{\infty}^{u}$ is a 2 -subcategory of ${ }^{u} A_{\infty}$, whose class of objects consists of all unital $A_{\infty}$-categories, 1-morphisms are all unital $A_{\infty}$-functors, and 2-morphisms are equivalence classes of all natural $A_{\infty}$-transformations between such functors. 
8.4 Proposition. Let $\mathcal{A}$ be an $A_{\infty}$-category. The strict 2-functor $A_{\infty}\left(\mathcal{A},{ }_{-}\right)$maps a unital $A_{\infty}$-category $\mathrm{C}$ to the unital $A_{\infty}$-category $A_{\infty}(\mathcal{A}, \mathrm{C})$, and a unital functor to a unital functor. Its restrictions $A_{\infty}\left(\mathcal{A},_{-}\right):{ }^{u} A_{\infty} \rightarrow{ }^{u} A_{\infty}, A_{\infty}\left(\mathcal{A},{ }_{-}\right): A_{\infty}^{u} \rightarrow A_{\infty}^{u}$ preserve 1-units and 2-units, thus, they are 2-functors in the usual sense.

Proof. Proposition 7.7 shows that $A_{\infty}(\mathcal{A}, \mathcal{C})$ is unital, if $\mathcal{C}$ is unital. If $g: \mathcal{B} \rightarrow \mathcal{C}$ is a unital $A_{\infty}$-functor between unital $A_{\infty}$-categories $\mathcal{B}$ and $\mathcal{C}$, then $\mathbf{i}^{\mathcal{B}} g \equiv g \mathbf{i}^{\mathfrak{C}}$ implies

$$
\left(1 \otimes \mathbf{i}^{\mathcal{B}}\right) M(1 \otimes g) M=\left(1 \otimes \mathbf{i}^{\mathcal{B}} g\right) M \equiv\left(1 \otimes g \mathbf{i}^{\mathfrak{C}}\right) M=(1 \otimes g) M\left(1 \otimes \mathbf{i}^{\mathrm{C}}\right) M,
$$

hence, $(1 \otimes g) M$ is unital. The fact, that $A_{\infty}\left(\mathcal{A},{ }_{-}\right)$preserves 1 -units and 2 -units is already proven in Proposition 7.7.

8.5. Categories modulo homotopy. $\mathcal{K}$-categories form a 2-category $\mathcal{K}$-Cat. We consider also non-unital $\mathcal{K}$-categories. They form a 2 -category $\mathcal{K}$-Cat ${ }^{n u}$ without 2-units (but with 1-units - identity functors).

8.6 Proposition. There is a strict 2-functor $\mathrm{k}: A_{\infty} \rightarrow \mathcal{K}$-Cat ${ }^{n u}$ of non-2-unital 2 -categories, which assigns to an $A_{\infty}$-category $\mathcal{C}$ the $\mathcal{K}$-category ke with the same class of objects $\mathrm{ObkC}=\mathrm{Ob} \mathcal{C}$, the same graded $\mathbb{k}$-module of morphisms $\mathrm{kC}(X, Y)=$ $\mathfrak{C}(X, Y)$, equipped with the differential $m_{1}$. Composition in $\mathrm{kC}$ is given by (the homotopy equivalence class of $) m_{2}: \mathcal{C}(X, Y) \otimes \mathfrak{C}(Y, Z) \rightarrow \mathcal{C}(X, Z)$. To an $A_{\infty}$-functor $f: \mathcal{A} \rightarrow \mathcal{B}$ is assigned $\mathrm{k} f: \mathrm{k} \mathcal{A} \rightarrow \mathrm{kB}$ such that $\mathrm{Obk} f=f: \mathrm{Ob} \mathcal{A} \rightarrow \mathrm{Ob} \mathcal{B}$, and for each pair of objects $X, Y$ of $\mathcal{A}$ we have $\mathrm{k} f=s f_{1} s^{-1}: \mathcal{A}(X, Y) \rightarrow \mathcal{B}(X f, Y f)$. To a natural $A_{\infty}$-transformation $r: f \rightarrow g: \mathcal{A} \rightarrow \mathcal{B}$ is assigned $\mathrm{k} r=r_{0} s^{-1}: \mathrm{k} f \rightarrow \mathrm{k} g$, that is, for each object $X$ of $\mathcal{A}$ the component ${ }_{X} \mathrm{k} r$ is the homotopy equivalence class of chain map ${ }_{X} r_{0} s^{-1}: \mathbb{k} \rightarrow \mathcal{B}(X f, X g)$. Unital $A_{\infty}$-categories and unital $A_{\infty}$-functors are mapped by $\mathrm{k}$ to unital $\mathcal{K}$-categories and unital $\mathcal{K}$-functors. The restriction $\mathrm{k}: A_{\infty}^{u} \rightarrow \mathcal{K}$-Cat is a 2-functor, which preserves 1-units and 2-units.

Proof. The identity (7.1.1) shows that $m_{2}$ is associative in $\mathcal{K}$. The identity

$$
(\mathrm{k} f \otimes \mathrm{k} f) m_{2}+(s \otimes s) f_{2} s^{-1} m_{1}+\left(1 \otimes m_{1}+m_{1} \otimes 1\right)(s \otimes s) f_{2} s^{-1}+m_{2} \mathrm{k} f=0
$$

shows that $\mathrm{k} f$ preserves the multiplication in $\mathcal{K}$.

The map ${ }_{X} \mathrm{k} r$ is a chain map since ${ }_{X} r_{0} s^{-1} m_{1}={ }_{X} r_{0} b_{1} s^{-1}=0$. If $r \equiv p: f \rightarrow$ $g: \mathcal{A} \rightarrow \mathcal{B}$, then ${ }_{X} r_{0}={ }_{X} p_{0}+{ }_{X} v_{0} b_{1}$ for some ${ }_{X} v_{0} \in(s \mathcal{B})^{-2}(X f, X g)$, therefore, ${ }_{X} r_{0} s^{-1}={ }_{X} p_{0} s^{-1}+\left({ }_{X} v_{0} s^{-1}\right) m_{1}$ and chain maps ${ }_{X} r_{0} s^{-1}$ and ${ }_{X} p_{0} s^{-1}$ are homotopic to each other, that is, $k r=k p$. The identity

$$
\begin{aligned}
& 0=s\left[\left(f_{1} \otimes_{Y} r_{0}\right) b_{2}+\left({ }_{X} r_{0} \otimes g_{1}\right) b_{2}+r_{1} b_{1}+b_{1} r_{1}\right] s^{-1} \\
= & \left(\mathrm{k} f \otimes_{Y} \mathrm{k} r\right) m_{2}-(X \mathrm{k} r \otimes \mathrm{k} g) m_{2}+s r_{1} s^{-1} m_{1}+m_{1} s r_{1} s^{-1}: \mathcal{A}(X, Y) \rightarrow \mathcal{B}(X f, Y g)
\end{aligned}
$$

shows that the following diagram commutes in $\mathcal{K}$ for all objects $X, Y$ of $\mathcal{A}$

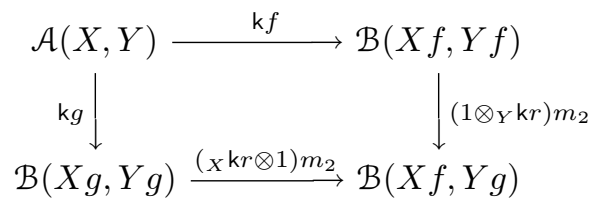


Thus $\mathrm{k} r$ is, indeed, a $\mathcal{K}$-natural transformation.

One checks easily that the composition of functors is preserved, and the both compositions of 1-morphisms and 2-morphisms are preserved. The vertical composition of 2-morphisms is preserved due to the property

$$
{ }_{X} \mathrm{k}\left[(r \otimes p) B_{2}\right]={ }_{X}\left[(r \otimes p) B_{2}\right]_{0} s^{-1}=\left({ }_{X} r_{0} \otimes X_{X} p_{0}\right) b_{2} s^{-1}=\left({ }_{X} \mathrm{k} r \otimes{ }_{X} \mathrm{k} p\right) m_{2} .
$$

Let $\mathcal{B}$ be a unital category. Then $\mathrm{k} \mathcal{B}$ is a unital $\mathcal{K}$-category. Indeed, for each object $X$ of $\mathcal{B}$ consider the corresponding element $1_{X}={ }_{X} \mathbf{i}_{0}^{\mathcal{B}} s^{-1}={ }_{X} \mathbf{k i}^{\mathcal{B}}: \mathbb{k} \rightarrow \mathcal{B}^{0}(X, X)$. Then for each pair $X, Y$ of objects of $\mathcal{C}$ the following equations hold in $\mathcal{K}$

$$
\begin{gathered}
\left(1 \otimes 1_{Y}\right) m_{2}=s\left(1 \otimes 1_{Y} s\right) b_{2} s^{-1}=s\left(1 \otimes{ }_{Y} \mathbf{i}_{0}^{\mathcal{B}}\right) b_{2} s^{-1}=s s^{-1} \\
=1: \mathcal{B}(X, Y) \rightarrow \mathcal{B}(X, Y), \\
\left(1_{X} \otimes 1\right) m_{2}=-s\left(1_{X} s \otimes 1\right) b_{2} s^{-1}=-s\left({ }_{X} \mathbf{i}_{0}^{\mathcal{B}} \otimes 1\right) b_{2} s^{-1} \\
=s s^{-1}=1: \mathcal{B}(X, Y) \rightarrow \mathcal{B}(X, Y) .
\end{gathered}
$$

That is, $1_{X}$ is the unit endomorphism of $X$.

If $f: \mathcal{A} \rightarrow \mathcal{C}$ is unital then, applying $\mathrm{k}$ to the equivalence $\mathbf{i}^{\mathcal{A}} f \equiv f \mathbf{i}^{\mathfrak{C}}$, we find that $\left(1_{\mathrm{id}_{\mathrm{k} \mathcal{A}}}\right) \mathrm{k} f=(\mathrm{k} f) 1_{\mathrm{id}_{\mathrm{ke}}}=1_{\mathrm{k} f}$, that is, $\mathrm{k} f$ is unital (it maps units into units).

8.7 Lemma (Cancellation). Let $\phi: \mathcal{C} \rightarrow \mathcal{B}$ be an $A_{\infty}$-functor, such that for all objects $X, Y$ of $\mathcal{C}$ the chain map $\phi_{1}:\left(s \mathfrak{C}(X, Y), b_{1}\right) \rightarrow\left(s \mathcal{B}(X \phi, Y \phi), b_{1}\right)$ is invertible in $\mathcal{K}$. Let $f, g: \mathcal{A} \rightarrow \mathcal{C}$ be $A_{\infty}$-functors. Let $y: f \phi \rightarrow g \phi: \mathcal{A} \rightarrow \mathcal{B}$ be a natural $A_{\infty}$-transformation. Then there is a unique up to equivalence natural $A_{\infty}$-transformation $t: f \rightarrow g: \mathcal{A} \rightarrow \mathcal{C}$ such that $y \equiv t \phi$.

Proof. First we prove the existence. We are looking for a 2-morphism $t: f \rightarrow g$ : $\mathcal{A} \rightarrow \mathcal{C}$ and a 3-morphism $v: y \rightarrow t \phi: f \phi \rightarrow g \phi: \mathcal{A} \rightarrow \mathcal{B}$. We have to satisfy the equations

$$
t b+b t=0, \quad y-t \phi=v b-b v .
$$

Let us construct the components of $t$ and $v$ by induction. Given a non-negative integer $n$, assume that we have already found components $t_{m}, v_{m}$ of the sought $t$, $v$ for $m<n$, such that the equations

$$
\begin{gathered}
(t b)_{m}+(b t)_{m}=0: s \mathcal{A}\left(X_{0}, X_{1}\right) \otimes \cdots \otimes s \mathcal{A}\left(X_{m-1}, X_{m}\right) \rightarrow s \mathfrak{C}\left(X_{0} f, X_{m} g\right), \quad \text { (8.7.1) } \\
y_{m}-(t \phi)_{m}=(v b-b v)_{m}: s \mathcal{A}\left(X_{0}, X_{1}\right) \otimes \cdots \otimes s \mathcal{A}\left(X_{m-1}, X_{m}\right) \rightarrow s \mathcal{B}\left(X_{0} f \phi, X_{m} g \phi\right),
\end{gathered}
$$

are satisfied for all $m<n$. Introduce an $(f, g)$-coderivation $\tilde{t}: T s \mathcal{A} \rightarrow T s \mathcal{C}$ of degree -1 by its components $\left(t_{0}, \ldots, t_{n-1}, 0,0, \ldots\right)$ and an $(f \phi, g \phi)$-coderivation $\tilde{v}: T s \mathcal{A} \rightarrow T s \mathcal{B}$ of degree -2 by its components $\left(v_{0}, \ldots, v_{n-1}, 0,0, \ldots\right)$. Define an $(f, g)$-coderivation $\lambda=\tilde{t} b+b \tilde{t}$ of degree 0 and an $(f \phi, g \phi)$-coderivation $\nu=$ $y-\tilde{t} \phi-\tilde{v} b+b \tilde{v}$ of degree -1 . Then equations (8.7.1), (8.7.2) imply that $\lambda_{m}=0$, $\nu_{m}=0$ for $m<n$. The identity $\lambda b-b \lambda=0$ implies that

$$
\lambda_{n} d=\lambda_{n} b_{1}-\sum_{\alpha+1+\beta=n}\left(1^{\otimes \alpha} \otimes b_{1} \otimes 1^{\otimes \beta}\right) \lambda_{n}=0 .
$$


The identity

$$
\nu b+b \nu=-\tilde{t} \phi b-b \tilde{t} \phi=-\lambda \phi
$$

implies that

$$
\nu_{n} d=\nu_{n} b_{1}+\sum_{\alpha+1+\beta=n}\left(1^{\otimes \alpha} \otimes b_{1} \otimes 1^{\otimes \beta}\right) \nu_{n}=-\lambda_{n} \phi_{1} .
$$

Denote $N=s \mathcal{A}\left(X_{0}, X_{1}\right) \otimes_{\mathbb{k}} \cdots \otimes_{\mathbb{k}} s \mathcal{A}\left(X_{n-1}, X_{n}\right)$, and consider the chain map

$$
u=\operatorname{Hom}\left(N, \phi_{1}\right): \operatorname{Hom}^{\bullet}\left(N, s \mathcal{C}\left(X_{0} f, X_{n} g\right)\right) \rightarrow \operatorname{Hom}^{\bullet}\left(N, s \mathcal{C}\left(X_{0} f \phi, X_{n} g \phi\right)\right) .
$$

Since $\phi_{1}$ is homotopy invertible, the map $u$ is homotopy invertible as well. Therefore, the complex Cone $(u)$ is acyclic. Moreover, it is contractible by Lemma Appendix B.1. Equations (8.7.3) and (8.7.4) in the form $-\lambda_{n} d=0, \nu_{n} d+\lambda_{n} \phi_{1}=0$ imply that

$$
\left(\nu_{n}, \lambda_{n}\right) \in \operatorname{Hom}_{\mathbb{k}}^{-1}\left(N, s \mathcal{B}\left(X_{0} f \phi, X_{n} g \phi\right)\right) \oplus \operatorname{Hom}_{\mathbb{k}}^{0}\left(N, s \mathcal{C}\left(X_{0} f, X_{n} g\right)\right)=\operatorname{Cone}^{-1}(u)
$$

is a boundary of some element

$$
\left(v_{n}, t_{n}\right) \in \operatorname{Hom}_{\mathbb{k}}^{-2}\left(N, s \mathcal{B}\left(X_{0} f \phi, X_{n} g \phi\right)\right) \oplus \operatorname{Hom}_{\mathbb{k}}^{-1}\left(N, s \mathcal{C}\left(X_{0} f, X_{n} g\right)\right)=\operatorname{Cone}^{-2}(u),
$$

that is, $v_{n} d+t_{n} u=\nu_{n}$ and $-t_{n} d=\lambda_{n}$. In other words, equations (8.7.1), (8.7.2) are satisfied for $m=n$, and we prove the existence of $t$ with the required properties by induction.

Now we prove the uniqueness of $t$. Assume that we have 2-morphisms $t, t^{\prime}: f \rightarrow$ $g: \mathcal{A} \rightarrow \mathcal{C}$ and 3-morphisms $v: y \rightarrow t \phi: f \phi \rightarrow g \phi: \mathcal{A} \rightarrow \mathcal{B}, v^{\prime}: y \rightarrow t^{\prime} \phi: f \phi \rightarrow$ $g \phi: \mathcal{A} \rightarrow \mathcal{B}$. We look for a 3-morphism $w$ and a 4-morphism $x$ :

$$
\begin{gathered}
w: t \rightarrow t^{\prime}: f \rightarrow g: \mathcal{A} \rightarrow \mathcal{C}, \\
x: v^{\prime} \rightarrow v+w \phi: y \rightarrow t^{\prime} \phi: f \phi \rightarrow g \phi: \mathcal{A} \rightarrow \mathcal{B} .
\end{gathered}
$$

They have to satisfy equations

$$
t-t^{\prime}=w b-b w, \quad v^{\prime}-v-w \phi=x b+b x .
$$

Let us construct the components of $w$ and $x$ by induction. Given a non-negative integer $n$, assume that we have already found components $w_{m}$ and $x_{m}$ of the sought $w, x$ for $m<n$, such that the equations

$$
\begin{gathered}
t_{m}-t_{m}^{\prime}=(w b-b w)_{m}: s \mathcal{A}\left(X_{0}, X_{1}\right) \\
\otimes \cdots \otimes s \mathcal{A}\left(X_{m-1}, X_{m}\right) \rightarrow s \mathcal{C}\left(X_{0} f, X_{m} g\right), \\
v_{m}^{\prime}-v_{m}-(w \phi)_{m}=(x b+b x)_{m}: s \mathcal{A}\left(X_{0}, X_{1}\right) \\
\otimes \cdots \otimes s \mathcal{A}\left(X_{m-1}, X_{m}\right) \rightarrow s \mathcal{B}\left(X_{0} f \phi, X_{m} g \phi\right),
\end{gathered}
$$

are satisfied for all $m<n$. Introduce an $(f, g)$-coderivation $\tilde{w}: T s \mathcal{A} \rightarrow T s \mathcal{C}$ of degree -2 by its components $\left(w_{0}, \ldots, w_{n-1}, 0,0, \ldots\right)$ and an $(f \phi, g \phi)$-coderivation $\tilde{x}: T s \mathcal{A} \rightarrow T s \mathcal{B}$ of degree -3 by its components $\left(x_{0}, \ldots, x_{n-1}, 0,0, \ldots\right)$. Define an 
$(f, g)$-coderivation $\lambda=t^{\prime}-t+\tilde{w} b+b \tilde{w}$ of degree -1 and an $(f \phi, g \phi)$-coderivation $\nu=v^{\prime}-v-\tilde{w} \phi-\tilde{x} b-b \tilde{x}$ of degree -2 . Then equations (8.7.5), (8.7.6) imply that $\lambda_{m}=0, \nu_{m}=0$ for $m<n$. The identity $\lambda b+b \lambda=0$ implies that

$$
\lambda_{n} d=\lambda_{n} b_{1}+\sum_{\alpha+1+\beta=n}\left(1^{\otimes \alpha} \otimes b_{1} \otimes 1^{\otimes \beta}\right) \lambda_{n}=0 .
$$

The identity

$$
\nu b-b \nu=v^{\prime} B_{1}-v B_{1}-\tilde{w} \phi b+b \tilde{w} \phi=y-t^{\prime} \phi-y+t \phi-\tilde{w} b \phi+b \tilde{w} \phi=-\lambda \phi
$$

implies that

$$
\nu_{n} d=\nu_{n} b_{1}-\sum_{\alpha+1+\beta=n}\left(1^{\otimes \alpha} \otimes b_{1} \otimes 1^{\otimes \beta}\right) \nu_{n}=-\lambda_{n} \phi_{1} .
$$

Hence,

$$
\left(\nu_{n}, \lambda_{n}\right) \in \operatorname{Hom}_{\mathbb{k}}^{-2}\left(N, s \mathcal{B}\left(X_{0} f \phi, X_{n} g \phi\right)\right) \oplus \operatorname{Hom}_{\mathbb{k}}^{-1}\left(N, s \mathcal{C}\left(X_{0} f, X_{n} g\right)\right)=\operatorname{Cone}^{-2}(u)
$$

is a cycle, therefore, it is a boundary of an element

$\left(x_{n}, w_{n}\right) \in \operatorname{Hom}_{\mathbb{k}}^{-3}\left(N, s \mathcal{B}\left(X_{0} f \phi, X_{n} g \phi\right)\right) \oplus \operatorname{Hom}_{\mathbb{k}}^{-2}\left(N, s \mathcal{C}\left(X_{0} f, X_{n} g\right)\right)=\operatorname{Cone}^{-3}(u)$, that is, $x_{n} d+w_{n} \phi_{1}=\nu_{n}$ and $-w_{n} d=\lambda_{n}$. In other words, equations (8.7.5), (8.7.6), are satisfied for $m=n$, and we prove the uniqueness of $t$, using induction.

A version of the following theorem is proved by Fukaya [Fuk, Theorem 8.6] with a different notion of unitality and under the additional assumption that the $\mathbb{k}$-modules $\mathcal{B}(W, Z), \mathcal{C}(X, Y)$ are free.

8.8 Theorem. Let $\mathcal{C}$ be an $A_{\infty}$-category and let $\mathcal{B}$ be a unital $A_{\infty}$-category. Let $\phi: \mathcal{C} \rightarrow \mathcal{B}$ be an $A_{\infty}$-functor such that for all objects $X, Y$ of $\mathcal{C}$ the chain map $\phi_{1}:\left(s \mathcal{C}(X, Y), b_{1}\right) \rightarrow\left(s \mathcal{B}(X \phi, Y \phi), b_{1}\right)$ is invertible in $\mathcal{K}$. Let $h: \mathrm{Ob} \mathcal{B} \rightarrow \mathrm{Ob} \mathcal{C}$ be a mapping. Assume that for each object $U$ of $\mathcal{B}$ the $\mathbb{k}$-linear maps

$$
\begin{aligned}
{ }_{U} r_{0}: \mathbb{k} & \rightarrow(s \mathcal{B})^{-1}(U, U h \phi), & { }_{U} p_{0}: \mathbb{k} \rightarrow(s \mathcal{B})^{-1}(U h \phi, U), \\
{ }_{U} w_{0}: \mathbb{k} \rightarrow(s \mathcal{B})^{-2}(U, U h \phi), & & { }_{U} v_{0}: \mathbb{k} \rightarrow(s \mathcal{B})^{-2}(U h \phi, U)
\end{aligned}
$$

are given such that

$$
\begin{gathered}
{ }_{U} r_{0} b_{1}=0, \quad{ }_{U} p_{0} b_{1}=0, \\
\left({ }_{U} r_{0} \otimes_{U} p_{0}\right) b_{2}-{ }_{U} \mathbf{i}_{0}^{\mathcal{B}}={ }_{U} w_{0} b_{1}, \\
\left({ }_{U} p_{0} \otimes_{U} r_{0}\right) b_{2}-{ }_{U h \phi} \mathbf{i}_{0}^{\mathcal{B}}={ }_{U} v_{0} b_{1} .
\end{gathered}
$$

Then there is an $A_{\infty}$-functor $\psi: \mathcal{B} \rightarrow \mathcal{C}$ such that $\mathrm{Ob} \psi=h$, there are natural $A_{\infty}$-transformations $r: \mathrm{id}_{\mathcal{B}} \rightarrow \psi \phi, p: \psi \phi \rightarrow \mathrm{id}_{\mathcal{B}}$ such that their 0-th components are the given ${ }_{U} r_{0}, U p_{0}$. Moreover, $r$ and $p$ are inverse to each other in the sense that

$$
(r \otimes p) B_{2} \equiv \mathbf{i}^{\mathcal{B}}, \quad(p \otimes r) B_{2} \equiv \psi \phi \mathbf{i}^{\mathcal{B}} .
$$

There exist unique up to equivalence natural $A_{\infty}$-transformations $t: \operatorname{id}_{\mathcal{C}} \rightarrow \phi \psi$, $q: \phi \psi \rightarrow \operatorname{id}_{\mathcal{e}}$ such that $t \phi \equiv \phi r: \phi \rightarrow \phi \psi \phi$ and $q \phi \equiv \phi p: \phi \psi \phi \rightarrow \phi$. 
Finally, $\mathcal{C}$ is unital with the unit

$$
\mathbf{i}^{\mathrm{e}}=(t \otimes q) B_{2}: \operatorname{id}_{\mathcal{C}} \rightarrow \operatorname{id}_{\mathcal{C}}: \mathcal{C} \rightarrow \mathcal{C},
$$

$\phi$ and $\psi$ are unital $A_{\infty}$-equivalences, quasi-inverse to each other via mutually inverse isomorphisms $r$ and $p, t$ and $q$ (in particular, $(q \otimes t) B_{2} \equiv \phi \psi \mathbf{i}^{\mathrm{e}}$ ).

Proof. We have to satisfy the equations

$$
\psi b=b \psi, \quad r b+b r=0 .
$$

We already know the map $\mathrm{Ob} \psi$ and the component $r_{0}$. Let us construct the remaining components of $\psi$ and $r$ by induction. Given a positive integer $n$, assume that we have already found components $\psi_{m}, r_{m}$ of the sought $\psi, r$ for $m<n$, such that the equations

$$
\begin{aligned}
& (\psi b)_{m}+(b \psi)_{m}=0: s \mathcal{B}\left(X_{0}, X_{1}\right) \otimes \cdots \otimes s \mathcal{B}\left(X_{m-1}, X_{m}\right) \rightarrow s \mathcal{C}\left(X_{0} h, X_{m} h\right) \\
& (r b+b r)_{m}=0: s \mathcal{B}\left(X_{0}, X_{1}\right) \otimes \cdots \otimes s \mathcal{B}\left(X_{m-1}, X_{m}\right) \rightarrow s \mathcal{B}\left(X_{0}, X_{m} h \phi\right)
\end{aligned}
$$

are satisfied for all $m<n$. Introduce a cocategory homomorphism $\tilde{\sim}: T s \mathcal{B} \rightarrow T s \mathcal{C}$ of degree 0 by its components $\left(\psi_{1}, \ldots, \psi_{n-1}, 0,0, \ldots\right)$ and a $\left(\mathrm{id}_{\mathcal{B}}, \tilde{\psi} \phi\right)$-coderivation $\tilde{r}: T s \mathcal{B} \rightarrow T s \mathcal{B}$ of degree -1 by its components $\left(r_{0}, r_{1}, \ldots, r_{n-1}, 0,0, \ldots\right)$. Define a $(\tilde{\psi}, \tilde{\psi})$-coderivation $\lambda=\tilde{\psi} b-b \tilde{\psi}$ of degree 1 and a map $\nu=-\tilde{r} b-b \tilde{r}+(\tilde{r} \otimes \lambda \phi) \theta$ : $T s \mathcal{B} \rightarrow T s \mathcal{B}$ of degree 0 . The commutator $\tilde{r} b+b \tilde{r}$ has the following property:

$$
(\tilde{r} b+b \tilde{r}) \Delta=\Delta[1 \otimes(\tilde{r} b+b \tilde{r})+(\tilde{r} b+b \tilde{r}) \otimes \tilde{\psi} \phi+\tilde{r} \otimes \lambda \phi] .
$$

By Proposition 3.1 the map $(\tilde{r} \otimes \lambda \phi) \theta$ has a similar property

$$
(\tilde{r} \otimes \lambda \phi) \theta \Delta=\Delta[1 \otimes(\tilde{r} \otimes \lambda \phi) \theta+(\tilde{r} \otimes \lambda \phi) \theta \otimes \tilde{\psi} \phi+\tilde{r} \otimes \lambda \phi] .
$$

Taking the difference we find that $\nu$ is an $\left(\operatorname{id}_{\mathcal{B}}, \tilde{\psi} \phi\right)$-coderivation. Equations (8.8.2), (8.8.3) imply that $\lambda_{m}=0, \nu_{m}=0$ for $m<n$ (the image of $(\tilde{r} \otimes \lambda \phi) \theta$ is contained in $T \geqslant 2 s \mathcal{B}$ ).

The identity $\lambda b+b \lambda=0$ implies that

$$
\lambda_{n} d=\lambda_{n} b_{1}+\sum_{\alpha+1+\beta=n}\left(1^{\otimes \alpha} \otimes b_{1} \otimes 1^{\otimes \beta}\right) \lambda_{n}=0 .
$$

The identity

$$
\nu b-b \nu=(\tilde{r} \otimes \lambda \phi) \theta b-b(\tilde{r} \otimes \lambda \phi) \theta
$$

implies that

$$
\nu_{n} b_{1}-\sum_{\alpha+1+\beta=n}\left(1^{\otimes \alpha} \otimes b_{1} \otimes 1^{\otimes \beta}\right) \nu_{n}=-\left(r_{0} \otimes \lambda_{n} \phi_{1}\right) b_{2}=-\lambda_{n} \phi_{1}\left(r_{0} \otimes 1\right) b_{2} .
$$

Set $N=s \mathcal{B}\left(X_{0}, X_{1}\right) \otimes_{\mathbb{k}} \cdots \otimes_{\mathbb{k}} s \mathcal{B}\left(X_{n-1}, X_{n}\right)$, and introduce a chain map

$u=\operatorname{Hom}\left(N, \phi_{1}\left(r_{0} \otimes 1\right) b_{2}\right): \operatorname{Hom}^{\bullet}\left(N, s \mathcal{C}\left(X_{0} h, X_{n} h\right)\right) \rightarrow \operatorname{Hom}^{\bullet}\left(N, s \mathcal{B}\left(X_{0}, X_{n} h \phi\right)\right)$.

Since $\phi_{1}$ and $\left(r_{0} \otimes 1\right) b_{2}$ are homotopy invertible by Lemma 7.14, the map $u$ is homotopy invertible as well. Therefore, the complex $\operatorname{Cone}(u)$ is contractible by 
Lemma Appendix B.1. Equations (8.8.4) and (8.8.5) in the form $-\lambda_{n} d=0, \nu_{n} d+$ $\lambda_{n} u=0$ imply that

$$
\left(\nu_{n}, \lambda_{n}\right) \in \operatorname{Hom}_{\mathrm{k}}^{0}\left(N, s \mathcal{B}\left(X_{0}, X_{n} h \phi\right)\right) \oplus \operatorname{Hom}_{\mathbb{k}}^{1}\left(N, s \mathcal{C}\left(X_{0} h, X_{n} h\right)\right)=\operatorname{Cone}^{0}(u)
$$

is a cycle. Hence, it is a boundary of some element

$$
\left(r_{n}, \psi_{n}\right) \in \operatorname{Hom}_{\mathbb{k}}^{-1}\left(N, s \mathcal{B}\left(X_{0}, X_{n} h \phi\right)\right) \oplus \operatorname{Hom}_{\mathbb{k}}^{0}\left(N, s \mathcal{C}\left(X_{0} h, X_{n} h\right)\right)=\operatorname{Cone}^{-1}(u),
$$

that is, $r_{n} d+\psi_{n} \phi_{1}\left(r_{0} \otimes 1\right) b_{2}=\nu_{n}$ and $-\psi_{n} d=\lambda_{n}$. In other words, equations (8.8.2), (8.8.3) are satisfied for $m=n$, and we prove the existence of $\psi$ and $r$ by induction.

Since $r_{0}$ and $p_{0}$ are homotopy inverse to each other in the sense of (8.8.1), we find by Proposition 7.15 that there exists a natural $A_{\infty}$-transformation $p: \psi \phi \rightarrow \mathrm{id}_{\mathcal{B}}$ such that $r$ and $p$ are inverse to each other.

The existence of $t, q$ such that $t \phi \equiv \phi r$ and $q \phi \equiv \phi p$ follows by Lemma 8.7. Let us prove that $\mathbf{i}^{\mathrm{e}}=(t \otimes q) B_{2}$ is a unit of $\mathcal{C}$. Due to Lemma 7.14 the maps $\left(r_{0} \otimes 1\right) b_{2}$, $\left(1 \otimes r_{0}\right) b_{2},\left(p_{0} \otimes 1\right) b_{2},\left(1 \otimes p_{0}\right) b_{2}$ are homotopy invertible. Let $f$ denote a homotopy inverse map of $\phi_{1}: s \mathcal{C}(X, Y) \rightarrow s \mathcal{B}(X \phi, Y \phi)$. The identity $t \phi \equiv \phi r$ implies that ${ }_{X} t_{0} \phi_{1}={ }_{X \phi} r_{0}+\kappa b_{1}$. Hence,

$$
\left(x \phi_{0} r_{0} \otimes 1\right) b_{2} \sim\left({ }_{x} t_{0} \phi_{1} \otimes 1\right) b_{2} \sim f\left({ }_{x} t_{0} \otimes 1\right) b_{2} \phi_{1} .
$$

Therefore, $\left(x t_{0} \otimes 1\right) b_{2} \sim \phi_{1}\left(x \phi r_{0} \otimes 1\right) b_{2} f$ is homotopy invertible. Similarly,

$$
\left(1 \otimes_{Y \phi} r_{0}\right) b_{2} \sim\left(1 \otimes_{Y} t_{0} \phi_{1}\right) b_{2} \sim f\left(1 \otimes_{Y} t_{0}\right) b_{2} \phi_{1}
$$

implies that $\left(1 \otimes_{Y} t_{0}\right) b_{2} \sim \phi_{1}\left(1 \otimes_{Y \phi} r_{0}\right) b_{2} f$ is homotopy invertible. Similarly, $\left(X q_{0} \otimes\right.$ 1) $b_{2}$ and $\left(1 \otimes_{Y} q_{0}\right) b_{2}$ are homotopy invertible.

The computation made in (7.14.1) shows that the product of the above homotopy invertible maps

$$
\left(q_{0} \otimes 1\right) b_{2}\left(t_{0} \otimes 1\right) b_{2} \sim-\left(t_{0} \otimes q_{0} \otimes 1\right)\left(b_{2} \otimes 1\right) b_{2}=-\left(\mathbf{i}_{0}^{\mathrm{e}} \otimes 1\right) b_{2}
$$

is the map we are studying. Similarly,

$$
\left(1 \otimes t_{0}\right) b_{2}\left(1 \otimes q_{0}\right) b_{2} \sim\left(1 \otimes t_{0} \otimes q_{0}\right)\left(1 \otimes b_{2}\right) b_{2}=\left(1 \otimes \mathbf{i}_{0}^{\mathrm{e}}\right) b_{2} .
$$

We conclude that both $\left(\mathbf{i}_{0}^{\mathrm{e}} \otimes 1\right) b_{2}$ and $\left(1 \otimes \mathbf{i}_{0}^{\mathrm{e}}\right) b_{2}$ are homotopy invertible.

Let us prove that $\left(\mathbf{i}^{\mathrm{e}} \otimes \mathbf{i}^{\mathrm{e}}\right) B_{2} \equiv \mathbf{i}^{\mathrm{e}}$. Due to Proposition 7.1 we have

$$
\mathbf{i}^{\mathfrak{e}} \phi=(t \otimes q) B_{2} \phi \equiv(t \phi \otimes q \phi) B_{2} \equiv(\phi r \otimes \phi p) B_{2}=\phi(r \otimes p) B_{2} \equiv \phi \mathbf{i}^{\mathcal{B}} .
$$

Using Proposition 7.1 again we get

$$
\left(\mathbf{i}^{\mathcal{C}} \otimes \mathbf{i}^{\mathrm{C}}\right) B_{2} \phi \equiv\left(\mathbf{i}^{\mathrm{C}} \phi \otimes \mathbf{i}^{\mathrm{C}} \phi\right) B_{2} \equiv\left(\phi \mathbf{i}^{\mathcal{B}} \otimes \phi \mathbf{i}^{\mathcal{B}}\right) B_{2}=\phi\left(\mathbf{i}^{\mathcal{B}} \otimes \mathbf{i}^{\mathcal{B}}\right) B_{2} \equiv \phi \mathbf{i}^{\mathcal{B}} \equiv \mathbf{i}^{\mathcal{C}} \phi .
$$

By Lemma 8.7 we deduce that $\left(\mathbf{i}^{\mathrm{e}} \otimes \mathbf{i}^{\mathrm{e}}\right) B_{2} \equiv \mathbf{i}^{\mathrm{e}}$, therefore, $\mathbf{i}^{\mathrm{e}}$ is a unit of $\mathcal{C}$.

Let us prove that $t$ and $q$ are inverse to each other. By definition, $(t \otimes q) B_{2}=\mathbf{i}^{\mathrm{e}}$. Due to Proposition 7.1

$$
(q \otimes t) B_{2} \phi \equiv(q \phi \otimes t \phi) B_{2} \equiv(\phi p \otimes \phi r) B_{2}=\phi(p \otimes r) B_{2} \equiv \phi \psi \phi \mathbf{i}^{\mathcal{B}} \equiv \phi \psi \mathbf{i}^{\mathrm{e}} \phi .
$$

By Lemma $8.7(q \otimes t) B_{2} \equiv \phi \psi \mathbf{i}^{\mathbf{e}}$. Hence, $t$ and $q$ are inverse to each other, as well as $r$ and $p$. Therefore, $\phi$ and $\psi$ are equivalences, quasi-inverse to each other. 
Relation (8.8.6) shows that $\phi$ is unital. Let us prove that $\psi$ is unital. We know that $\psi \phi$ is isomorphic to the identity functor. Thus, $\psi \phi$ is unital by (8.2.4). Hence, $\mathbf{i}^{\mathcal{B}} \psi \phi \equiv \psi \phi \mathbf{i}^{\mathcal{B}} \equiv \psi \mathbf{i}^{\mathfrak{C}} \phi$. By Lemma 8.7 we have $\mathbf{i}^{\mathcal{B}} \psi \equiv \psi \mathbf{i}^{\mathrm{e}}$, and $\psi$ is unital. The theorem is proven.

8.9 Corollary. Let $\mathcal{C}, \mathcal{B}$ be unital $A_{\infty}$-categories, and let $\phi: \mathcal{C} \rightarrow \mathcal{B}$ be an equivalence. Then $\phi$ is unital.

Proof. Since $\phi$ is an equivalence, $\mathrm{k} \phi$ is an equivalence as well. Hence, $\phi_{1}$ is invertible in $\mathcal{K}$. There exists an $A_{\infty}$-functor $\psi: \mathcal{B} \rightarrow \mathcal{C}$ quasi-inverse to $\phi$, and mutually inverse isomorphisms $r: \mathrm{id}_{\mathcal{B}} \rightarrow \psi \phi, p: \psi \phi \rightarrow \mathrm{id}_{\mathcal{B}}$. In particular, the assumptions of Theorem 8.8 are satisfied by $\phi, \mathrm{Ob} \psi: \mathrm{Ob} \mathcal{B} \rightarrow \mathrm{ObC}, r_{0}$ and $p_{0}$. The theorem implies that $\phi$ is unital.

8.10 Corollary. Let $\mathcal{C}$ be an $A_{\infty}$-algebra and let $\mathcal{B}$ be a unital $A_{\infty}$-algebra (viewed as $A_{\infty}$-categories with one object). Let $\phi: \mathcal{C} \rightarrow \mathcal{B}$ be an $A_{\infty}$-homomorphism such that $\phi_{1}:\left(s \mathcal{C}, b_{1}\right) \rightarrow\left(s \mathcal{B}, b_{1}\right)$ is homotopy invertible. Then $\mathcal{C}$ and $\phi$ are unital, and $\phi$ is an $A_{\infty}$-equivalence.

Existence of $\phi$ with the above property might be taken as an equivalence relation on the class of unital $A_{\infty}$-algebras.

8.11. Strictly unital $A_{\infty}$-categories. A strict unit of an object $X$ of an $A_{\infty}$-category $\mathcal{A}$ is an element $1_{X} \in \mathcal{A}^{0}(X, X)$, such that $\left(f \otimes 1_{X}\right) m_{2}=f$, $\left(1_{X} \otimes g\right) m_{2}=g$, whenever these make sense, and $\left(\cdots \otimes 1_{X} \otimes \ldots\right) m_{n}=0$ if $n \neq 2$ (see e.g. [FOOO, Fuk, Kel01]). We may write it as a map $1_{X}: \mathbb{k} \rightarrow \mathcal{A}(X, X)$, $1 \mapsto 1_{X}$. Assume that $\mathcal{A}$ has a strict unit for each object $X$. For example, a differential graded category $\mathcal{A}$ has strict units. Then we introduce a coderivation $\mathbf{i}^{\mathcal{A}}: \operatorname{id}_{\mathcal{A}} \rightarrow \operatorname{id}_{\mathcal{A}}: \mathcal{A} \rightarrow \mathcal{A}$, whose components are $\mathbf{i}_{0}^{\mathcal{A}}: \mathbb{k} \rightarrow s \mathcal{A}(X, X)$, $1 \mapsto 1_{X} s=X_{X} \mathbf{i}_{0}^{\mathcal{A}}$, and $\mathbf{i}_{k}^{\mathcal{A}}=0$ for $k>0$. The conditions on $1_{X}$ imply that $\left(1 \otimes \mathbf{i}_{0}^{\mathcal{A}}\right) b_{2}=1: s \mathcal{A}(Y, X) \rightarrow s \mathcal{A}(Y, X)$ and $\left(\mathbf{i}_{0}^{\mathcal{A}} \otimes 1\right) b_{2}=-1: s \mathcal{A}(X, Z) \rightarrow s \mathcal{A}(X, Z)$. One deduces that $\mathbf{i}^{\mathcal{A}}$ is a natural $A_{\infty}$-transformation. If an $A_{\infty}$-category $\mathcal{A}$ has two such transformations - strict units $\mathbf{i}$ and $\mathbf{i}^{\prime}$, then they must coincide because of the above equations. We call $\mathcal{A}$ strictly unital if it has a strict unit $\mathbf{i}^{\mathcal{A}}$. Naturally, a strictly unital $A_{\infty}$-category is unital.

For any $A_{\infty}$-functor $f: \mathcal{C} \rightarrow \mathcal{A}$ the natural $A_{\infty}$-transformation $1_{f} s=f \mathbf{i}^{\mathcal{A}}: f \rightarrow$ $f: \mathcal{C} \rightarrow \mathcal{A}$ has the components ${ }_{X}\left(f \mathbf{i}^{\mathcal{A}}\right)_{0}={ }_{X f} \mathbf{i}_{0}^{\mathcal{A}}: \mathbb{k} \rightarrow s \mathcal{A}(X f, X f)$ and $\left(f \mathbf{i}^{\mathcal{A}}\right)_{k}=0$ for $k>0$. It is the unit 2-endomorphism of $f$.

If $A_{\infty}$-category $\mathcal{B}$ is strictly unital, then so is $\mathcal{C}=A_{\infty}(\mathcal{A}, \mathcal{B})$ for an arbitrary $A_{\infty}$-category $\mathcal{A}$. Indeed, for an arbitrary $A_{\infty}$-functor $f: \mathcal{A} \rightarrow \mathcal{B}$ there is a unit 2-endomorphism $1_{f} s=f \mathbf{i}^{\mathcal{B}}: f \rightarrow f$. We set $\mathbf{i}_{0}^{\mathfrak{C}}: \mathbb{k} \rightarrow\left[s A_{\infty}(\mathcal{A}, \mathcal{B})\right]^{-1}(f, f), 1 \mapsto 1_{f} s$, and $\mathbf{i}_{k}^{\mathrm{e}}=0$ for $k>0$. For any element $r \in \mathcal{C}(g, f)$ we have $\left(r \otimes 1_{f} s\right) B_{2}=r$. For any element $p \in \mathcal{C}(f, h)$ we have $p\left(1_{f} s \otimes 1\right) B_{2}=p\left(\left(f \mathbf{i}^{\mathcal{B}}\right)_{0} \otimes 1\right) b_{2}=-p$. We have also $\mathbf{i}^{\mathrm{e}} B_{1}=0$ and $\left(\cdots \otimes \mathbf{i}^{\mathrm{e}} \otimes \ldots\right) B_{n}=0$ if $n>2$, due to (5.1.3). Therefore, $\mathbf{i}^{\mathrm{C}}$ satisfies the required conditions.

Another approach to $\mathrm{i}^{\mathrm{C}}$ uses the $A_{\infty}$-functor $M: T s A_{\infty}(\mathcal{A}, \mathcal{B}) \otimes T s A_{\infty}(\mathcal{B}, \mathcal{B}) \rightarrow$ $T s A_{\infty}(\mathcal{A}, \mathcal{B})=\mathcal{C}$. We have $\left(1 \otimes \mathrm{id}_{\mathcal{B}}\right) M=\operatorname{id}_{\mathcal{C}}$ by $(4.1 .3)$, and the natural $A_{\infty}$-transformations $\left(1 \otimes \mathbf{i}^{\mathcal{B}}\right) M$ and $\mathbf{i}^{\mathcal{C}}$ of ide coincide. Indeed, $\left[\left(1 \otimes \mathbf{i}^{\mathcal{B}}\right) M\right]_{0}: \mathbb{k} \rightarrow(s \mathcal{C})^{-1}(f, f)$, 
$1 \mapsto\left(f \mid \mathbf{i}^{\mathcal{B}}\right) M_{01}=f \mathbf{i}^{\mathcal{B}}={ }_{f} \mathbf{i}_{0}^{\mathbf{C}}$. For all $n \geqslant 0$ we have $\left[\left(1 \otimes \mathbf{i}^{\mathcal{B}}\right) M\right]_{n}: r^{1} \otimes \cdots \otimes r^{n} \mapsto$ $\left(r^{1} \otimes \cdots \otimes r^{n} \otimes \mathbf{i}^{\mathcal{B}}\right) M_{n 1}$. By (4.1.4) the components

$$
\left[\left(r^{1} \otimes \cdots \otimes r^{n} \otimes \mathbf{i}^{\mathcal{B}}\right) M_{n 1}\right]_{k}=\sum_{l}\left(r^{1} \otimes \cdots \otimes r^{n}\right) \theta_{k l} \mathbf{i}_{l}^{\mathcal{B}}=\left(r^{1} \otimes \cdots \otimes r^{n}\right) \theta_{k 0} \mathbf{i}_{0}^{\mathcal{B}}
$$

vanish for $n>0$.

8.12. Other examples of unital $A_{\infty}$-categories. More examples of unital categories might be obtained via Theorem 8.8. An $A_{\infty}$-category with a homotopy unit in the sense of Fukaya, Oh, Ohta and Ono [FOOO, Definition 20.1] clarified by Fukaya [Fuk, Definition 5.11] is also a unital category in our sense. Indeed, these authors enlarge given $A_{\infty}$-category $\mathcal{C}$ to a strictly unital $A_{\infty}$-category $\mathcal{B}$ by adding extra elements to $\mathcal{C}(X, X)$, so that the natural embedding $\left(\mathcal{C}(X, Y), m_{1}\right) \hookrightarrow\left(\mathcal{B}(X, Y), m_{1}\right)$ were a homotopy equivalence. Setting $r_{0}=p_{0}=\mathbf{i}_{0}^{\mathcal{B}}$ we view the above situation as a particular case of Theorem 8.8.

If an $A_{\infty}$-functor $\phi: \mathcal{C} \rightarrow \mathcal{B}$ to a unital $A_{\infty}$-category $\mathcal{B}$ is invertible, then $\mathcal{C}$ is unital. Indeed, since there exists an $A_{\infty}$-functor $\psi: \mathcal{B} \rightarrow \mathcal{C}$ such that $\phi \psi=\operatorname{id} \mathcal{e}$ and $\psi \phi=\operatorname{id}_{\mathcal{B}}$, then the map $\phi_{1}$ is invertible with inverse $\psi_{1}$. The remaining data are $\mathrm{Ob} \psi: \mathrm{ObB} \rightarrow \mathrm{ObC}$ and ${ }_{X} r_{0}={ }_{X} p_{0}={ }_{X} \mathbf{i}_{0}^{\mathcal{B}}: \mathbb{k} \rightarrow s \mathcal{B}(X, X)$. Since $\left(\mathbf{i}^{\mathcal{B}} \otimes \mathbf{i}^{\mathcal{B}}\right) B_{2} \equiv \mathbf{i}^{\mathcal{B}}$ we have $\left({ }_{X} \mathbf{i}_{0}^{\mathcal{B}} \otimes_{X} \mathbf{i}_{0}^{\mathcal{B}}\right) b_{2}-{ }_{X} \mathbf{i}_{0}^{\mathcal{B}} \in \operatorname{Im} b_{1}$ and conditions (8.8.1) are satisfied. The data constructed in Theorem 8.8 will be precisely $\psi: \mathcal{B} \rightarrow \mathcal{C}$ and $r=p=\mathbf{i}^{\mathcal{B}}$. Since $\phi$ is unital by Theorem 8.8, we may choose $\mathbf{i}^{\mathrm{C}}=\phi \mathbf{i}^{\mathcal{B}} \psi$ as a unit of $\mathcal{C}$.

If a unital $A_{\infty}$-category $\mathcal{C}$ is equivalent to a strictly unital $A_{\infty}$-category $\mathcal{B}$ via an $A_{\infty}$-functor $\phi: \mathcal{C} \rightarrow \mathcal{B}$, then $(1 \otimes \phi) M: A_{\infty}(\mathcal{A}, \mathcal{C}) \rightarrow A_{\infty}(\mathcal{A}, \mathcal{C})$ is also an equivalence for an arbitrary $A_{\infty}$-category $\mathcal{A}$ as Proposition 8.4 shows. Thus, a unital $A_{\infty}$-category $A_{\infty}(\mathcal{A}, \mathcal{C})$ is equivalent to a strictly unital $A_{\infty}$-category $A_{\infty}(\mathcal{A}, \mathcal{B})$. In particular, if $\phi$ is invertible, then $(1 \otimes \phi) M$ is invertible as well.

8.13. Cohomology of $A_{\infty}$-categories. Using a lax monoidal functor from $\mathcal{K}$ to some monoidal category we get another 2 -functor, which can be composed with k. For instance, there is a cohomology functor $H^{\bullet}: \mathcal{K} \rightarrow \mathbb{Z}$-grad-k-mod, which induces a 2-functor $H^{\bullet}: \mathcal{K}$-Cat $\rightarrow \mathbb{Z}$-grad-k-Cat. In practice we will use the 0 -th cohomology functor $H^{0}: \mathcal{K} \rightarrow \mathbb{k}$-mod, the corresponding 2-functor $H^{0}: \mathcal{K}$-Cat $\rightarrow$ $\mathbb{k}$ - $\mathrm{C} a t$, and the composite 2 -functor

$$
A_{\infty}^{u} \stackrel{\mathrm{k}}{\longrightarrow} \mathcal{K}-\mathrm{C} a t \stackrel{H^{0}}{\longrightarrow} \mathbb{k}-\mathrm{C} a t,
$$

which is also denoted by $H^{0}$. It takes a unital $A_{\infty}$-category $\mathcal{C}$ into a $\mathbb{k}$-linear category $H^{0}(\mathcal{C})$ with the same class of objects $\mathrm{Ob} H^{0}(\mathcal{C})=\mathrm{Ob} \mathcal{C}$. Its morphism space between objects $X$ and $Y$ is $H^{0}(\mathcal{C})(X, Y)=H^{0}\left(\mathcal{C}(X, Y), m_{1}\right)$, the 0 -th cohomology with respect to the differential $m_{1}=s b_{1} s^{-1}$. The composition in $H^{0}(\mathrm{C})$ is induced by $m_{2}$, and the units by $\mathbf{i}_{0}^{\mathrm{e}} s^{-1}$.

For example, the homotopy category $\mathrm{K}(\mathcal{A})$ of complexes of objects of an abelian category $\mathcal{A}$ is the 0 -th cohomology $H^{0}(\mathrm{C}(\mathcal{A})$ ) of the differential graded category of complexes $\mathrm{C}(\mathcal{A})$. 


\section{Appendix A. Enriched 2-categories}

Recall that $\mathscr{U}$ is a fixed universe. Let $\mathcal{V}=(\mathcal{V}, \otimes, c, \mathbb{1})$ be a symmetric monoidal $\mathscr{U}$-category (that is, all $\mathcal{V}(X, Y)$ are $\mathscr{U}$-small sets). In this article we shall use $(\mathcal{V}, \otimes, c, \mathbb{1})=\left(\mathbb{k}-\bmod , \otimes_{\mathbb{k}}, \sigma, \mathbb{k}\right)$, where $\sigma$ is the permutation isomorphism, or $(\mathcal{V}, \otimes, c, \mathbb{1})=\left(\mathcal{K}, \otimes_{\mathbb{k}}, c, \mathbb{k}\right)$, where $\mathcal{K}$ is the category of differential graded $\mathbb{k}$-modules, whose morphisms are chain maps modulo homotopy, and $c$ is its standard symmetry. There is a notion of a category $\mathcal{C}$ enriched in $\mathcal{V}(\mathcal{V}$-categories, $\mathcal{V}$-functors, $\mathcal{V}$-natural transformations), see Kelly [Kel82], summarized e.g. in [KL01]: for all objects $X$, $Y$ of $\mathcal{C} \mathrm{C}(X, Y)$ is an object of $\mathcal{V}$. Denote by $\mathcal{V}$ - $\mathrm{C} a t$ the category, whose objects are $\mathcal{V}$-categories and morphisms are $\mathcal{V}$-functors. Since $\mathcal{V}$ is symmetric, the category $\mathcal{V}$-Cat is symmetric monoidal with the tensor product $\mathcal{A} \otimes \mathcal{B}$ of $\mathcal{V}$-categories $\mathcal{A}, \mathcal{B}$ defined via $\mathrm{Ob}(\mathcal{A} \otimes \mathcal{B})=\mathrm{Ob} \mathcal{A} \times \mathrm{Ob} \mathcal{B}, \mathcal{A} \otimes \mathcal{B}(X \times Y, U \times V)=\mathcal{A}(X, U) \otimes \mathcal{B}(Y, V)$. Thus, we may consider the 1-category $\mathcal{V}$-Cat-Cat of $\mathcal{V}$-eat-categories and $\mathcal{V}$-Catfunctors. We may interpret it in the same way, as $\mathcal{C}$ at-Cat is interpreted as the category of 2-categories. So we say that objects of $\mathcal{V}$-Cat-Cat are $\mathcal{V}$-2-categories $\mathfrak{A}$, as defined below. To restore the definition of a usual 2-category, it suffices to take $\mathcal{V}=(\mathscr{U}$-Sets, $\times, \varnothing)$.

Appendix A.1 Definition ( $\mathcal{V}$-2-category). A 1-unital 2-unital $\mathcal{V}$-2-category $\mathfrak{A}$ consists of

- a class of objects Ob $\mathfrak{A}$;

- for any pair of objects $\mathcal{A}, \mathcal{B} \in \mathrm{Ob} \mathfrak{A}$ a $\mathcal{V}$-category $\mathfrak{A}(\mathcal{A}, \mathcal{B})$;

- for any object $\mathcal{A} \in \mathrm{Ob} \mathfrak{A}$ a $\mathcal{V}$-functor $\underline{\mathbb{1}} \rightarrow \mathfrak{A}(\mathcal{A}, \mathcal{A}), 1 \mapsto \operatorname{id}_{\mathcal{A}}$;

- for any triple $\mathcal{A}, \mathcal{B}, \mathcal{C}$ of objects of $\mathfrak{A}$ a $\mathcal{V}$-functor

$$
\mathfrak{A}(\mathcal{A}, \mathcal{B}) \otimes \mathfrak{A}(\mathcal{B}, \mathcal{C}) \rightarrow \mathfrak{A}(\mathcal{A}, \mathcal{C}), \quad(f, g) \mapsto f g,
$$

such that the following $\mathcal{V}$-functors are equal (modulo the associativity isomorphism in $\mathcal{V}): f$ id $=f=$ id $f, f(g h)=(f g) h$.

Here the unit $\mathcal{V}$-category $\underline{\mathbb{1}}$ has the set of objects Ob $\underline{\mathbb{1}}=\{1\}$, and $\underline{\mathbb{1}}(1,1)=\mathbb{1}$ is the unit object of $\mathcal{V}$. The above definition has an equivalent unpacked form, namely, Definition Appendix A.3. We also need generalizations of the above $\mathcal{V}$-2-categories - 1-unital non-2-unital $\mathcal{V}$-2-categories, which contain unit 1-morphisms, but do not contain unit 2-morphisms. An expanded definition of the latter follows. It seems that it does not have a concise version.

Appendix A.2 Definition (Non-2-unital V-2-category). A 1-unital non-2unital $\mathcal{V}$-2-category $\mathfrak{A}$ consists of

- a class of objects $\mathrm{Ob} \mathfrak{A}$;

- a class of 1-morphisms $\mathfrak{A}(\mathcal{A}, \mathcal{B})$ for any pair $\mathcal{A}, \mathcal{B}$ of objects of $\mathfrak{A}$;

- an object of 2-morphisms $\mathfrak{A}(\mathcal{A}, \mathcal{B})(f, g) \in \mathrm{Ob} \mathcal{V}$ for any pair of 1-morphisms $f, g \in \mathfrak{A}(\mathcal{A}, \mathcal{B})$

- a strictly associative composition of 1-morphisms $\mathfrak{A}(\mathcal{A}, \mathcal{B}) \times \mathfrak{A}(\mathcal{B}, \mathcal{C}) \rightarrow$ $\mathfrak{A}(\mathcal{A}, \mathcal{C}),(f, g) \mapsto f g ;$ 
- a strict two-sided unit 1-morphism $\operatorname{id}_{\mathcal{A}} \in \mathfrak{A}(\mathcal{A}, \mathcal{A})$ for each object $\mathcal{A}$ of $\mathfrak{A}$;

- a right action of a 1-morphism $k: \mathcal{B} \rightarrow \mathcal{C}$ on 2-morphisms $\cdot k: \mathfrak{A}(\mathcal{A}, \mathcal{B})(f, g) \rightarrow$ $\mathfrak{A}(\mathcal{A}, \mathcal{C})(f k, g k) \in \operatorname{Mor} \mathcal{V}$

- a left action of a 1-morphism $e: \mathcal{D} \rightarrow \mathcal{A}$ on 2-morphisms $e \cdot: \mathfrak{A}(\mathcal{A}, \mathcal{B})(f, g) \rightarrow$ $\mathfrak{A}(\mathcal{D}, \mathcal{B})(e f, e g) \in \operatorname{Mor} \mathcal{V}$;

- a vertical composition of 2-morphisms $m_{2}: \mathfrak{A}(\mathcal{A}, \mathcal{B})(f, g) \otimes \mathfrak{A}(\mathcal{A}, \mathcal{B})(g, h) \rightarrow$ $\mathfrak{A}(\mathcal{A}, \mathcal{B})(f, h) \in \operatorname{Mor} \mathcal{V}$

such that

- $m_{2}$ is associative (in monoidal category $\mathcal{V}$ );

- the right and the left actions

(a) commute with each other:

$$
(e \cdot)(\cdot k)=(\cdot k)(e \cdot), \quad \text { for } \mathcal{D} \stackrel{e}{\longrightarrow} \mathcal{A} \underset{g}{\stackrel{f}{\longrightarrow}} \mathcal{B} \stackrel{k}{\longrightarrow} \mathcal{C},
$$

(b) are associative:

$$
\begin{aligned}
& (\cdot k)\left(\cdot k^{\prime}\right)=\cdot\left(k k^{\prime}\right), \quad \text { for } \mathcal{A} \underset{g}{\stackrel{f}{\longrightarrow}} \mathcal{B} \stackrel{k}{\longrightarrow} \mathcal{C} \stackrel{k^{\prime}}{\longrightarrow} \mathcal{D}, \\
& \left(e^{\prime} \cdot\right)(e \cdot)=\left(e^{\prime} e\right) \cdot, \quad \text { for } \mathcal{C} \stackrel{e^{\prime}}{\longrightarrow} \mathcal{D} \stackrel{e}{\longrightarrow} \mathcal{A} \underset{g}{\stackrel{f}{\longrightarrow}} \mathcal{B},
\end{aligned}
$$

(c) and unital: $\left(\cdot \operatorname{id}_{\mathcal{B}}\right)=\mathrm{id},\left(\operatorname{id}_{\mathcal{A}} \cdot\right)=\mathrm{id}$;

- the right and the left actions of 1-morphisms on 2-morphisms preserve the vertical composition:

$$
\begin{aligned}
& \mathfrak{A}(\mathcal{A}, \mathcal{B})(f, g) \otimes \mathfrak{A}(\mathcal{A}, \mathcal{B})(g, h) \stackrel{m_{2}}{\longrightarrow} \mathfrak{A}(\mathcal{A}, \mathcal{B})(f, h) \\
& (\cdot k) \otimes(\cdot k) \downarrow \quad \mid \cdot k \quad \text { for } \underset{\mathcal{A}}{\stackrel{-g}{\longrightarrow}} \mathcal{B} \stackrel{k}{\longrightarrow} \mathcal{C}, \\
& \mathfrak{A}(\mathcal{A}, \mathcal{C})(f k, g k) \otimes \mathfrak{A}(\mathcal{A}, \mathcal{C})(g k, h k) \stackrel{m_{2}}{\longrightarrow} \mathfrak{A}(\mathcal{A}, \mathcal{C})(f k, h k) \\
& \mathfrak{A}(\mathcal{A}, \mathcal{B})(f, g) \otimes \mathfrak{A}(\mathcal{A}, \mathcal{B})(g, h) \stackrel{m_{2}}{\longrightarrow} \mathfrak{A}(\mathcal{A}, \mathcal{B})(f, h) \\
& (e \cdot) \otimes(e \cdot) \mid \quad=\quad \text { e. } \quad \text { for } \mathcal{D} \stackrel{e}{\longrightarrow} \underset{\mathcal{A}}{\stackrel{f \rightarrow}{\longrightarrow}} \mathcal{B} ; \\
& \mathfrak{A}(\mathcal{D}, \mathcal{B})(e f, e g) \otimes \mathfrak{A}(\mathcal{D}, \mathcal{B})(e g, e h) \stackrel{m_{2}}{\longrightarrow} \mathfrak{A}(\mathcal{D}, \mathcal{B})(e f, e h)
\end{aligned}
$$

- the distributivity law holds:

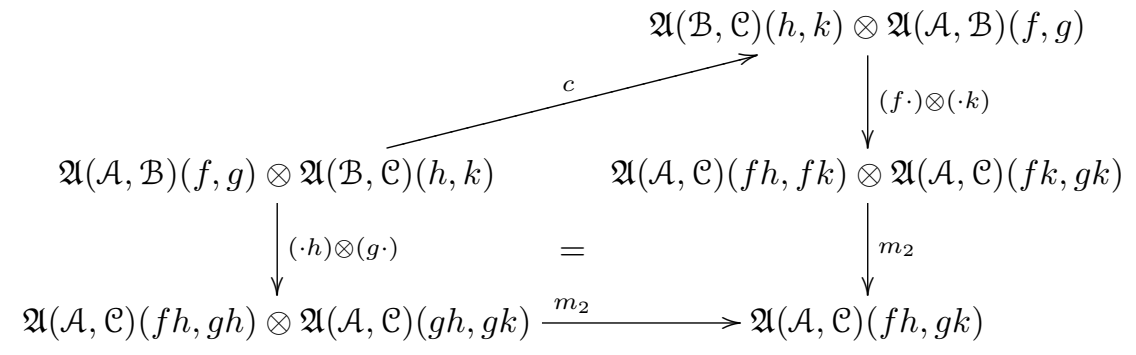




$$
\text { for } \mathcal{A} \underset{g}{\stackrel{f}{\rightleftarrows}} \mathcal{B} \underset{k}{\stackrel{h}{\rightleftarrows}} \mathcal{C} \text {. }
$$

The following definition is equivalent to Definition Appendix A.1.

Appendix A.3 Definition (2-unital V-2-category). A 1-unital 2-unital V-2category $\mathfrak{A}$ consists of the same data as in Definition Appendix A.2 plus a morphism $1_{f}: \mathbb{1} \rightarrow \mathfrak{A}(\mathcal{A}, \mathcal{B})(f, f)$ for any 1-morphism $f$, which is a two-sided unit with respect to $m_{2}$, such that homomorphisms $\cdot k, e \cdot$ preserve the units $1_{-}$.

\section{Appendix B. Contractibility}

One can avoid using the following lemma in this article. However, it might be used in order to replace inductive constructions with recurrent formulas.

Appendix B.1 Lemma. Let a chain map $u: A \rightarrow C$ be homotopically invertible. Then Cone $(u)$ is contractible.

Proof. The homotopy category $\mathcal{K}=\mathrm{K}(\mathbb{k}-\bmod )$ is triangulated and it has a distinguished triangle $A \stackrel{u}{\longrightarrow} C \stackrel{p}{\longrightarrow}$ Cone $(u) \stackrel{q}{\longrightarrow} A[1] \stackrel{u[1]}{\longrightarrow}$ (e.g. [Gri87, Corollaire 5.13]). Since $u p=0, q u[1]=0$ (e.g. [Gri87, Proposition 2.8]), and $u$ is invertible in $\mathcal{K}$, we deduce that $p=0$ and $q=0$ in $\mathcal{K}$. Since $\mathcal{K}\left(\operatorname{Cone}(u),{ }_{-}\right)$is a homological functor (e.g. [Gri87, Proposition 2.10]), we have $\mathcal{K}(\operatorname{Cone}(u)$, Cone $(u))=0$, that is, Cone $(u) \simeq 0$ in $\mathcal{K}$.

Let us construct an explicit homotopy between $\operatorname{id}_{\operatorname{Cone}(u)}$ and $0_{\text {Cone }(u)}$. There exists a chain map $v: C \rightarrow A$ homotopically inverse to $u$. That is, there are maps $h^{\prime}: A \rightarrow A, h^{\prime \prime}: C \rightarrow C$ of degree -1 such that $u v=1+h^{\prime} d^{A}+d^{A} h^{\prime}: A \rightarrow A$, $v u=1+h^{\prime \prime} d^{C}+d^{C} h^{\prime \prime}: C \rightarrow C$. Using the notation at the end of Section 1 we define a map $h$ : Cone $(u) \rightarrow$ Cone $(u)$ of degree -1 by the formula $(c, a) h=\left(-c h^{\prime \prime}, c v+a h^{\prime}\right)$, $(c, a) \in C^{k} \oplus A^{k+1}=\operatorname{Cone}^{k}(u)$. Let us compute the boundary of $h$ :

$$
\begin{aligned}
(c, a)(h d+d h)= & \left(-c h^{\prime \prime}, c v+a h^{\prime}\right) d+\left(c d^{C}+a u,-a d^{A}\right) h \\
= & \left(-c h^{\prime \prime} d^{C}+c v u+a h^{\prime} u,-c v d^{A}-a h^{\prime} d^{A}\right) \\
& +\left(-c d^{C} h^{\prime \prime}-a u h^{\prime \prime}, c d^{C} v+a u v-a d^{A} h^{\prime}\right) \\
= & \left(c+a h^{\prime} u-a u h^{\prime \prime}, a\right) .
\end{aligned}
$$

Hence, $h d+d h=1-f:$ Cone $(u) \rightarrow$ Cone $(u)$, where the map $f:$ Cone $(u) \rightarrow$ Cone $(u)$ is defined via $(c, a) f=\left(a u h^{\prime \prime}-a h^{\prime} u, 0\right)$. We conclude that $f$ is a chain map homotopic to the identity map. A sequence of equivalences $\operatorname{id}_{\operatorname{Cone}(u)} \sim f=$ $\operatorname{id}_{\text {Cone }(u)} f \sim f^{2}=0$ proves that $\operatorname{Cone}(u)$ is contractible. It gives also an explicit homotopy - the map $\underline{h}=h+h f:$ Cone $(u) \rightarrow$ Cone $(u)$ of degree -1 , which satisfies $\operatorname{id}_{\operatorname{Cone}(u)}=\underline{h} d+d \underline{h}$. This homotopy might be used to replace inductive constructions in this paper with recurrent formulas.

Acknowledgements. I am grateful to all the participants of the $A_{\infty}$-category seminar at the Institute of Mathematics, Kyiv, especially to Yu. Bespalov, O. Manzyuk, S. Ovsienko. 


\section{References}

[Bat98] M. A. Batanin, Monoidal globular categories as natural environment for the theory of weak n-categories, Adv. Math. 136 (1998), 39-103.

[Boa66] J. M. Boardman, The principle of signs, Enseignement Math. (2) 12 (1966), 191-194.

[Bou73] N. Bourbaki, Univers, SGA 4: Théorie des Topos et Cohomologie Etale des Schémas, Tome 1. Théorie des Topos, Lect. Notes in Math., vol. 269, Springer, Heidelberg, 1972-1973, appendice, pp. 185-217.

[Car48] H. Cartan, Sur la cohomologie des espaces où opère un groupe. Notions algébriques préliminaires, C. R. Acad. Sci. Paris 226 (1948), no. 2, 148150.

[Dri02] V. G. Drinfeld, DG quotients of DG categories, math.KT/0210114, 2002.

[FOOO] K. Fukaya, Y.-G. Oh, H. Ohta, and K. Ono, Lagrangian intersection Floer theory - anomaly and obstruction -, book in preparation.

[Fuk] K. Fukaya, Floer homology and mirror symmetry II, preprint.

[Fuk93] K. Fukaya, Morse homotopy, $A^{\infty}$-category, and Floer homologies, The Proceedings of the 1993 GARC Workshop on Geometry and Topology (H. J. Kim, ed.), Lecture Notes series, vol. 18, Seoul National University, 1993, 1-102.

[GJ90] E. Getzler and J. D. S. Jones, $A_{\infty}$-algebras and the cyclic bar complex, Illinois J. Math. 34 (1990), no. 2, 256-283.

[Gri87] P.-P. Grivel, Catégories dérivées et foncteurs dérivés, Algebraic Dmodules (J. Coates and S. Helgason, eds.), Perspectives in Mathematics, vol. 2, Academic Press, Boston, New York, London, Tokyo, 1987, pp. 1108.

[GV73] A. Grothendieck and J. L. Verdier, Préfaisceaux, SGA 4: Théorie des Topos et Cohomologie Etale des Schémas, Tome 1. Théorie des Topos, Lect. Notes in Math., vol. 269, Springer, Heidelberg, 1972-1973, pp. 1184.

[Kad80] T. V. Kadeishvili, On the theory of homology of fiber spaces, Uspekhi Mat. Nauk 35 (1980), no. 3, 183-188, Translated in Russ. Math. Surv. 35 (1980) no. 3, 231-238.

[Kad82] T. V. Kadeishvili, The algebraic structure in the homology of an $A(\infty)$ algebra, Soobshch. Akad. Nauk Gruzin. SSR 108 (1982), no. 2, 249-252, in Russian.

[Kel01] B. Keller, Introduction to A-infinity algebras and modules, Homology, Homotopy and Applications 3 (2001), no. 1, 1-35.

[Kel82] G. M. Kelly, Basic concepts of enriched category theory, London Math. Soc. Lecture Notes, vol. 64, Cambridge Univ. Press, Cambridge, 1982.

[KL01] T. Kerler and V. V. Lyubashenko, Non-semisimple topological quantum field theories for 3-manifolds with corners, Lect. Notes in Math., vol. 1765, Springer-Verlag, Heidelberg, 2001, vi+379 p. 
[Kon95] M. Kontsevich, Homological algebra of mirror symmetry, Proc. Internat. Cong. Math., Zürich, Switzerland 1994 (Basel), vol. 1, Birkhäuser Verlag, 1995, 120-139.

[KS02] M. Kontsevich and Yan Soibelman, $A_{\infty}$-categories and non-commutative geometry, in preparation, 2002.

[KS] M. Kontsevich and Yan Soibelman, Deformation theory, book in preparation.

[Kos47] J.-L. Koszul, Sur les opérateurs de dérivation dans un anneau, C. R. Acad. Sci. Paris 225 (1947), 217-219.

[LH02] K. Lefèvre-Hasegawa, Sur les $A_{\infty}$-catégories, Ph.D. thesis, in preparation, 2002.

[Lei02] T. Leinster, A survey of definitions of n-category, Theory Appl. Categ. 10 (2002), 1-70 (electronic).

[Mac63] S. Mac Lane, Homology, Die Grundlehren der mathematischen Wissenschaften, no. 114, Springer-Verlag, Berlin, Heidelberg, 1963.

[Smi80] V. A. Smirnov, Homology of fiber spaces, Uspekhi Mat. Nauk 35 (1980), no. 3, 227-230, Translated in Russ. Math. Surveys 35 (1980) no 3, 294298.

[Sta63] J. D. Stasheff, Homotopy associativity of H-spaces, I \& II, Trans. Amer. Math. Soc. 108 (1963), 275-292, 293-312.

This article may be accessed via WWW at http://www.rmi.acnet.ge/hha/ or by anonymous ftp at

ftp://ftp.rmi.acnet.ge/pub/hha/volumes/2003/n1a1/v5n1a1.(dvi,ps,pdf)

Volodymyr Lyubashenko lub@imath.kiev.ua

Institute of Mathematics,

National Academy of Sciences of Ukraine,

3 Tereshchenkivska st.,

Kyiv-4, 01601 MSP,

Ukraine 